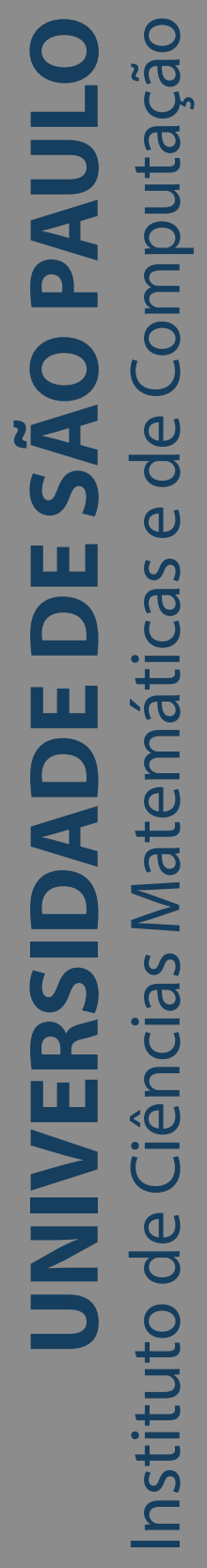

\title{
Visual analytics via graph signal processing
}

\section{Alcebíades Dal Col Júnior}

Tese de Doutorado do Programa de Pós-Graduação em Ciências de Computação e Matemática Computacional (PPG-CCMC) 

SERVIÇO DE PÓS-GRADUAÇÃO DO ICMC-USP

Data de Depósito:

Assinatura:

\section{Alcebíades Dal Col Júnior}

\section{Visual analytics via graph signal processing}

Doctoral dissertation submitted to the Institute of Mathematics and Computer Sciences - ICMC-USP, in partial fulfillment of the requirements for the degree of the Doctorate Program in Computer Science and Computational Mathematics. FINAL VERSION

Concentration Area: Computer Science and Computational Mathematics

Advisor: Prof. Dr. Luis Gustavo Nonato

\section{USP - São Carlos}

May 2018 
Ficha catalográfica elaborada pela Biblioteca Prof. Achille Bassi e Seção Técnica de Informática, ICMC/USP, com os dados inseridos pelo(a) autor(a)

Dal Col, Alcebiades Visual analytics via graph signal processing / Alcebiades Dal Col; orientador Luis Gustavo Nonato. -- São Carlos, 2018. $102 \mathrm{p}$.

Tese (Doutorado - Programa de Pós-Graduação em Ciências de Computação e Matemática Computacional) Instituto de Ciências Matemáticas e de Computação, Universidade de São Paulo, 2018.

1. Ciência da Computação. 2. Matemática da Computação. 3. Teoria dos grafos. 4. Wavelets em grafos. I. Nonato, Luis Gustavo, orient. II. Título.

Bibliotecários responsáveis pela estrutura de catalogação da publicação de acordo com a AACR2: Gláucia Maria Saia Cristianini - CRB - 8/4938 Juliana de Souza Moraes - CRB - 8/6176 


\section{Alcebíades Dal Col Júnior}

\section{Análise visual via processamento de signal em grafo}

Tese apresentada ao Instituto de Ciências Matemáticas e de Computação - ICMC-USP, como parte dos requisitos para obtenção do título de Doutor em Ciências - Ciências de Computação e Matemática Computacional. VERSÃO REVISADA

Área de Concentração: Ciências de Computação e Matemática Computacional

Orientador: Prof. Dr. Luis Gustavo Nonato

USP - São Carlos

Maio de 2018 

Aos meus familiares e amigos. 

Dedico esta tese,

À Deus, em primeiro lugar, pelo dom da vida, e pela inteligência, sabedoria, e saúde necessárias para desenvolver com sucesso o meu projeto de doutorado que culminou na presente tese.

À minha estimada mãe, Joicia Maria Pereira Dal Col, pela confiança depositada no seu filho, pelo incentivo ao desenvolvimento de todo o meu potencial, por acreditar na minha capacidade, pelas palavras de sabedoria e motivação em momentos de dificuldade, e por suportar com paciência a distância e a saudade.

Ao meu irmão, Cristiano Dal Col, primeiramente, por realizar uma viagem de carro de dezesseis horas para levar minha mudança para São Carlos, cidade onde realizei o doutorado, e por sempre dar apoio aos meus estudos.

Ao Prof. Dr. Fabiano Petronetto, por me impulsionar à realização do doutorado, mostrando sua real importância à minha carreira acadêmica, e por me aconselhar e acompanhar como a um verdadeiro filho com zelo e compreensão.

Aos meus amigos, Apoenã Passos, Flávio De Souza, Ginnara Mexia, Patricia Tempesta, Alex Carlucci, Natália Marques, Camila Rizzardi, Andreza Beezão, Mayron Moreira, Nelson Silva, Thaís Maria Dalbelo, Renato Andrielli, e Jaqueline Ferreira, pela calorosa acolhida em uma cidade completamente nova para mim, pelos almoços de domingo, e por serem como uma segunda família.

Às minhas queridas tias, Luíse Maria, Marli Maria, Heli Hela, e Márcia Maria, pelo amor incondicional, pelo apoio à minha família na minha ausência, sem o qual seria impossível o meu afastamento para a realização do doutorado, e pelas visitas em um cidade situada a aproximadamente mil quilômetros de suas casas.

À Paróquia Nossa Senhora de Fátima, na pessoal do Pe. João Roberto e demais amigos paroquianos, Sr. Sebastião, David Maldonado, Patricia Helena Maldonado, Luciana, e muitos outros, pelo acolhimento espiritual e amizade. 
À escola de idiomas CNA, pelo suporte incrível na utilização da língua inglesa.

Aos meus amigos de longa data, Rodrigo De Oliveira Kirmse e Camilo Campana, pela amizade, por sempre compartilharem seu tempo comigo, pelas conversas agradáveis que por vezes ajudaram a distrair de questões complicadas do doutorado, e por escutarem pacientemente e opinarem sobre diversas questões relacionadas ao meu doutorado.

Ao Prof. Dr. José Alberto Cuminato, pela ótima recomendação junto a Fundação de Amparo à Pesquisa do Estado de São Paulo (FAPESP), graças à realização do seu curso de Álgebra Linear Aplicada, recomendação que foi de suma importância para a concessão de uma bolsa e consequente migração de bolsas, da bolsa da Coordenação de Aperfeiçoamento de Pessoal de Nível Superior (CAPES) para a bolsa da FAPESP.

Ao Instituto de Ciências Matemáticas e de Computação (ICMC) e seus funcionários dedicados, pelo apoio técnico-administrativo, pela competência na realização de seu trabalho, pela paciência, e pelas orientações.

Ao coral do ICMC e ao maestro Sergio Alberto de Oliveira, pelas aulas de técnica vocal, e pelas gratificantes atividades extracurriculares, como apresentações ao ar livre no campus da universidade.

Ao Centro Acadêmico Armando de Salles Oliveira, por me permitir a prática do forró.

Aos membros titulares da minha banca de qualificação, Prof. Dr. Fernando Vieira Paulovich, Prof. Dr. Marcio Gameiro, Prof. Dr. Esdras Medeiros (Universidade Federal do Ceará UFC), e Prof. Dr. Luis Gustavo Nonato, pelas críticas construtivas e direcionamentos.

Aos meus amigos e colaboradores, Fabio Dias e Paola Valdivia, pela colaboração em artigos publicados em jornais internacionais, por estarem sempre dispostos a ajudar quando preciso, pelas sugestões, pelas dicas valiosas, e pelas horas de trabalho árduo.

Aos meus amigos do grupo de pesquisa, Prof. Dr. Marcio Gameiro, Prof. Dr. Afonso Paiva, Prof. Dr. Antonio Castelo, Prof. Dr. Luis Gustavo Nonato, Rodrigo Contreras, Markus Diego, Luzia Romanetto, Dalia Bonilla, Fabio Dias, Paola Valdivia, Douglas Cedrim, Danilo Andrade, Rafael Umino, José Filomen, e Lucas Pagliosa, por compartilharem seu conhecimento e experiências, além da apreciação do meu trabalho com inúmeras críticas construtivas que foram de grande importância para o desenvolvimento do meu projeto de doutorado.

Aos Profs. Drs. Marcio Gameiro e Afonso Paiva, pela supervisão do meu projeto de pesquisa junto a agência de fomento FAPESP durante o período de afastamento do meu orientador para estágio no exterior, e pela atenção e resposta rápida no tratamento de questões burocráticas. 
Ao meu orientador, Prof. Dr. Luis Gustavo Nonato, pelo empenho dedicado a minha formação acadêmica, por compartilhar o seu incrível talento para pesquisa científica e escrita de textos acadêmicos, e pela excelente orientação.

Ao Prof. Dr. Claudio T. Silva e Ann Messinger, administradora da Tandon School of Engineering, por oferecerem todo o suporte necessário para a realização de um estágio no exterior junto a New York University (NYU). Estágio que infelizmente não pode ser realizado devido à questões pessoais.

Aos membros titulares da minha banca de doutorado, Prof. Dr. Esdras Medeiros (UFC), Prof. Dr. Alexandre Falcão (Universidade Estadual de Campinas - UNICAMP), Prof. Dr. Afonso Paiva, e Prof. Dr. Luis Gustavo Nonato, por aceitarem prontamente a participação na minha defesa.

Às agências de fomento, CAPES e FAPESP (Processo 2015/03330-7), pelo financiamento ao longo do meu doutorado, possibilitando a minha dedicação integral aos estudos. 

"For what will it profit a man if he gains the whole world, and loses his own soul?"

(Mark 8:36)

"Pois, que adianta ao homem ganhar o mundo inteiro $e$ perder a sua alma?"

(Marcos 8:36) 



\section{ABSTRACT}

DAL COL, A. Visual analytics via graph signal processing. 2018. 102 p. Tese (Doutorado em Ciências - Ciências de Computação e Matemática Computacional) - Instituto de Ciências Matemáticas e de Computação, Universidade de São Paulo, São Carlos - SP, 2018.

The classical wavelet transform has been widely used in image and signal processing, where a signal is decomposed into a combination of basis signals. By analyzing the individual contribution of the basis signals, one can infer properties of the original signal. This dissertation presents an overview of the extension of the classical signal processing theory to graph domains. Specifically, we review the graph Fourier transform and graph wavelet transforms both of which based on the spectral graph theory, and explore their properties through illustrative examples. The main features of the spectral graph wavelet transforms are presented using synthetic and real-world data.

Furthermore, we introduce in this dissertation a novel method for visual analysis of dynamic networks, which relies on the graph wavelet theory. Dynamic networks naturally appear in a multitude of applications from different domains. Analyzing and exploring dynamic networks in order to understand and detect patterns and phenomena is challenging, fostering the development of new methodologies, particularly in the field of visual analytics. Our method enables the automatic analysis of a signal defined on the nodes of a network, making viable the detection of network properties. Specifically, we use a fast approximation of the graph wavelet transform to derive a set of wavelet coefficients, which are then used to identify activity patterns on large networks, including their temporal recurrence. The wavelet coefficients naturally encode spatial and temporal variations of the signal, leading to an efficient and meaningful representation. This method allows for the exploration of the structural evolution of the network and their patterns over time. The effectiveness of our approach is demonstrated using different scenarios and comparisons involving real dynamic networks.

Keywords: Graph signal processing, spectral graph wavelets, dynamic networks, visual analytics. 



\section{RESUMO}

DAL COL, A. Análise visual via processamento de signal em grafo. 2018. 102 p. Tese (Doutorado em Ciências - Ciências de Computação e Matemática Computacional) - Instituto de Ciências Matemáticas e de Computação, Universidade de São Paulo, São Carlos - SP, 2018.

A transformada wavelet clássica tem sido amplamente usada no processamento de imagens e sinais, onde um sinal é decomposto em uma combinação de sinais de base. Analisando a contribuição individual dos sinais de base, pode-se inferir propriedades do sinal original. Esta tese apresenta uma visão geral da extensão da teoria clássica de processamento de sinais para grafos. Especificamente, revisamos a transformada de Fourier em grafo e as transformadas wavelet em grafo ambas fundamentadas na teoria espectral de grafos, e exploramos suas propriedades através de exemplos ilustrativos. As principais características das transformadas wavelet espectrais em grafo são apresentadas usando dados sintéticos e reais.

Além disso, introduzimos nesta tese um método inovador para análise visual de redes dinâmicas, que utiliza a teoria de wavelets em grafo. Redes dinâmicas aparecem naturalmente em uma infinidade de aplicações de diferentes domínios. Analisar e explorar redes dinâmicas a fim de entender e detectar padrões e fenômenos é desafiador, fomentando o desenvolvimento de novas metodologias, particularmente no campo de análise visual. Nosso método permite a análise automática de um sinal definido nos vértices de uma rede, tornando possível a detecção de propriedades da rede. Especificamente, usamos uma aproximação da transformada wavelet em grafo para obter um conjunto de coeficientes wavelet, que são então usados para identificar padrões de atividade em redes de grande porte, incluindo a sua recorrência temporal. Os coeficientes wavelet naturalmente codificam variações espaciais e temporais do sinal, criando uma representação eficiente e com significado expressivo. Esse método permite explorar a evolução estrutural da rede e seus padrões ao longo do tempo. A eficácia da nossa abordagem é demonstrada usando diferentes cenários e comparações envolvendo redes dinâmicas reais.

Palavras-chave: Processamento de sinais em grafo, wavelets espectrais em grafo, redes dinâmicas, análise visual. 

Figure $1-$ Graph wavelet data analysis . . . . . . . . . . . . . 35

Figure 2 - Animation techniques . . . . . . . . . . . . 37

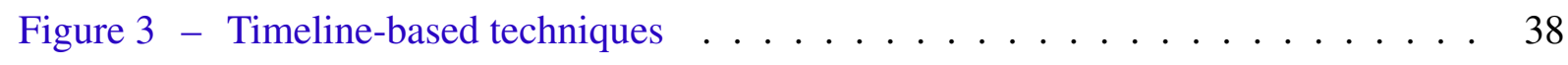

Figure 4 - Matrix-based techniques . . . . . . . . . . . . . 39

Figure 5 - Hadlak's method . . . . . . . . . . . . . . . 40

Figure 6 - GraphFlow . . . . . . . . . . . . . . . . 40

Figure 7 - Projection as support for dynamic network exploration . . . . . . . . . . 41

Figure 8 - Signal defined on the nodes of a graph . . . . . . . . . . . . 44

Figure 9 - Number of zero crossings for the path graph with 64 nodes . . . . . . . 45

Figure 10 - Signals of low frequency, high frequency, and $f_{0}$ defined on the path graph with 64 nodes . . . . . . . . . . . . . . . . . . . . . 4 47

Figure 11 - Scaling function and wavelet kernels . . . . . . . . . . . . . . 51

Figure $12-$ Graph wavelet transform of signal $f_{0} \ldots \ldots \ldots$. . . . . . . . 52

Figure 13 - Computational cost and error in the approximation of wavelet coefficients for a synthetic network . . . . . . . . . . . . . . . 54

Figure 14 - An example of graph with low and high frequency nodes . . . . . . . 55

Figure 15 - Dictionary of uniform translates . . . . . . . . . . . . . . 59

Figure 16 - Uniform translates adapting to graph Laplacian spectra . . . . . . . . . 59

Figure 17 - Sum of squares of the SGW and SAGW dictionaries for a path graph . . . . 61

Figure 18 - Comet graph and corresponding spectral distribution . . . . . . . . . . . 62

Figure 19 - Graph spectral filters of the SGW and SAGW dictionaries for a comet graph with 64 nodes . . . . . . . . . . . . . . . . . . 6 63

Figure 20 - Second largest graph Laplacian eigenvector defined on a comet graph with 64 nodes . . . . . . . . . . . . . . . . . 6 63

Figure $21-$ Minnesota road graph . . . . . . . . . . . . . . . . . 64

Figure 22 - Time-varying data modeled by the Cartesian product of two graphs . . . . 66

Figure 23 - Graphs: spatial $G$ and temporal $H \ldots \ldots$. . . . . . . . . . 66

Figure 24 - Wavelet coefficients of node $\tau_{a} l_{10}$ for signals $f_{i}, i=1,2,3,4 \ldots \ldots$. . . . 67

Figure 25 - Wavelet coefficients of node $\tau_{a} l_{10}$ for signal $f_{3}$ after edges removal . . . . 68

Figure 26 - Number of taxi pick-ups in downtown Manhattan on August 12, 2013 . . . 69

Figure 27 - Spatial and spatio-temporal wavelet coefficients of nodes on Manhattan corners 69

Figure 28 - Dynamic network model . . . . . . . . . . . . . . 72

Figure 29 - Torque analogy for node classification . . . . . . . . . . . . . 74 
Figure 30 - Node classification performed on the example from Figure $14 \ldots$. . . . 75

Figure 31 - Proposed interface for dynamic network exploration . . . . . . . . . 76

Figure 32 - Node classification for the synthetic dynamic network . . . . . . . . . . 77

Figure 33 - RSP and TimeArcs methods applied to the synthetic dynamic network . . . 78

Figure 34 - Neighborhood of node six in the synthetic dynamic network in time slices 3 and $4 \ldots \ldots \ldots \ldots \ldots \ldots$. . . . . . . . . . . . . . . . . . . . . . . . . .

Figure 35 - Network analyzer for the high school dataset . . . . . . . . . . . . 81

Figure 36 - Signal and node classification for the high school dataset on the first Monday at $9: 24,10: 24$, and $14: 30 \ldots \ldots \ldots$. . . . . . . . . . . . 82

Figure 37 - Comparison against the RSP method considering the high school dataset . . 84

Figure 38 - TimeArcs method applied to the high school dataset . . . . . . . . . 85

Figure 39 - Interface considering taxi trips in Manhattan in December 2009 . . . . . . . 86

Figure 40 - Node time series of East Village, Lower East Side, and Theater District zones 86

Figure 41 - Interface considering taxi trips in Manhattan in December 2009 on the period between December 24th and December 27th . . . . . . . . . . . 87

Figure 42 - Multidimensional projection of Iris dataset via regularization on graphs . . . 91 
$\overline{2}$

Table 1 - Journal citation metrics according to IEEE Xplore . . . . . . . . . . 30

Table 2 - Full text views of the publications directly related to the dissertation . . . . 90

Table $3-$ General details of Iris dataset . . . . . . . . . . . . . . . 92 



\section{LIST OF ABBREVIATIONS AND ACRONYMS}

CAPES Coordenação de Aperfeiçoamento de Pessoal de Nível Superior

CiSE Computing in Science \& Engineering

FAPESP Fundação de Amparo à Pesquisa do Estado de São Paulo

GSPBOX Graph Signal Processing Toolbox

ICMC Instituto de Ciências Matemáticas e de Computação

MDS Multidimensional Scaling

MSV Massive Sequence Views

NYC New York City

NYU New York University

RSP Reducing Snapshots to Points

SAGW Spectrum-adapted graph wavelets

SGW Spectral graph wavelets

TLC Taxi and Limousine Commission

TVCG Transactions on Visualization and Computer Graphics

UFC Universidade Federal do Ceará

UNICAMP Universidade Estadual de Campinas 

$G, H$ - Graphs

$V$ - Node set

$n$ - Number of graph nodes

$\tau, \imath-$ Graph nodes

$E$ - Edge set

$W$ - Adjacency matrix

L - Graph Laplacian

$D$ - Degree matrix

$u$ - Eigenvector

$\lambda$ - Eigenvalue

$\mathscr{L}$ - Normalized graph Laplacian

$f$ - Signal

$Z_{G}-$ Set of zero crossings

$\hat{f}$ - Graph Fourier transform of $f$

$\star$ — Convolution operator

$T^{s}$ - Graph wavelet operator

$\hat{g}$ - Kernel or graph spectral filter

$s-$ Scale

$\psi_{s, \tau}$ - Graph wavelet in node $\tau$ at scale $s$

$\delta_{\tau}$ - Delta signal

$U$ - Matrix whose columns are the graph Laplacian eigenvectors

$D_{\hat{g}}$ - Diagonal matrix with entries $d_{i i}=\hat{g}\left(s \lambda_{i}\right)$

$W_{f}(s, \tau)$ - Wavelet coefficient in node $\tau$ at scale $s$

$\hat{g}(s \lambda)$ - Wavelet kernel

$\hat{h}$ - Scaling function 
$S_{f}-$ Scaling function coefficient

$T_{k}$ - Chebyshev polynomials

$\bar{T}_{k}$ - Shifted Chebyshev polynomials

$|E|$ - Number of edges in $G$

$\Lambda-\left\{\lambda_{1}, \lambda_{2}, \ldots, \lambda_{n}\right\}$, Spectral domain

$\left\{\hat{g}_{m}\right\}_{m=1,2, \ldots, M}$ - Dictionary of graph spectral filters

$M-$ Number of graph spectral filters

$\left\{\Gamma_{k}\right\}-$ Set of functions

$A, B$ - Frame bounds

$G-$ Sum of squares of the graph spectral filters

$R_{1}, R_{2}, R_{3}-$ Regions on the Minnesota road graph

$I_{j}-$ Set of indexes corresponding to interval $\left[a_{j}, b_{j}\right]$

$N$ - Number of time slices

$Z-\{1,2, \ldots, N\}$

$F V_{\tau}$ - Wavelet coefficient feature vector

$F$ - Smooth signal that approximates the signal $f$ 
INTRODUCTION ........................ 27

$1.1 \quad$ Contributions of the Dissertation . . . . . . . . . . . . . . 28

1.2 Publications Directly Related to the Dissertation . . . . . . . . . 29

$1.3 \quad$ Dissertation Outline . . . . . . . . . . . . . . . . 30

2 REVIEW OF THE LITERATURE . . . . . . . . . . . . . 33

$2.1 \quad$ Graph Signal Processing \& Graph Wavelets . . . . . . . . . . . . 33

$2.2 \quad$ Visual Analytics of Dynamic Networks . . . . . . . . . . . . 36

3 SPECTRAL GRAPH THEORY . . . . . . . . . . . . . . 43

$3.1 \quad$ Basic Definitions . . . . . . . . . . . . . . 43

$3.2 \quad$ Graph Fourier Transform . . . . . . . . . . . . . . . . 45

3.3 Spectral Graph Wavelet Transform . . . . . . . . . . . . . . 47

3.4 Fast Spectral Graph Wavelet Transform . . . . . . . . . . . . . . 52

4 ILLUSTRATIVE INTERPRETATION OF GRAPH WAVELETS . . . 57

$4.1 \quad$ Spectrum-Adapted Graph Wavelet Transform . . . . . . . . . . 58

4.2 Practical Differences between the Spectral Graph Wavelet Transforms ... . . . . . . . . . . . . . . . . 62

4.3 Interplay among Topology, Spectrum, and Wavelet Coefficients . . 64

$4.4 \quad$ Time-Varying Data Analysis via Graph Wavelets . . . . . . . . . 65

$5 \quad$ VISUAL ANALYSIS OF DYNAMIC NETWORKS . . . . . . . 71

$5.1 \quad$ Dynamic Network Model . . . . . . . . . . . . . . . 72

$5.2 \quad$ Node Signal \& Edge Weights . . . . . . . . . . . . . . . 73

$5.3 \quad$ Node Classification . . . . . . . . . . . . . . . 74

$5.4 \quad$ From Wavelet Coefficients to Visualization . . . . . . . . . . . 75

$5.5 \quad$ Usage Scenarios . . . . . . . . . . . . . . . 80

$5.6 \quad$ Final Considerations \& Discussion . . . . . . . . . . . 88

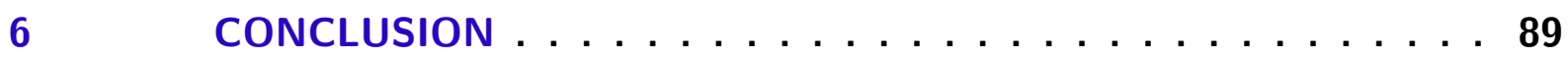

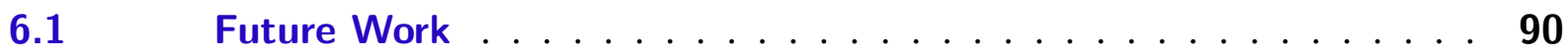


CHAPTER

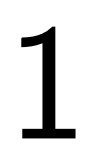

1

\section{INTRODUCTION}

The emerging field of signal processing on graphs has been growing substantially in the last years. The whole theory is based on irregular structures called graphs. Any data whose elements have relations with each other can be modeled as a graph, which makes the theory's applicability immense. Elements are named graph nodes and relations are edges. The ability to analyze a signal defined on such an irregular environment is a remarkable characteristic of this theory, extending the classical signal processing on regular domains to a more complex scenario. Applications are diverse, such as image processing, semi-supervised learning, interpersonal contact data analysis, just to name a few.

Graph signal processing has a well-established theoretical and computational framework. The theory formulation is strongly based on mathematical principles with the concern of being computationally efficient. Most of the tools developed have fast, low-memory versions, supporting the use in large data (HAMMOND; VANDERGHEYNST; GRIBONVAL, 2011; SHUMAN; VANDERGHEYNST; FROSSARD, 2011), which is of unprecedented importance today since we are living the advent of new technologies, our ability to acquire, store, and transmit data is huge and grows amazingly (HILBERT; LÓPEZ, 2011).

In more practical terms, when working with the tools of graph signal processing, the construction of the graph and choice of an appropriate signal is of paramount importance. For example, in an image denoising application, the graph can be built considering pixels as graph nodes and the gray level as signal (SHUMAN et al., 2013). Thus, a noisy image can be filtered via graph regularization, a technique employed to smooth a signal defined on a graph. In urban data analysis, the street map of a city can be seen as a graph, where street connections represent graph nodes and the number of taxi pick-ups in their neighborhood represents the signal (VALDIVIA et al., 2015). In this context, the spectral graph wavelets can be used to infer signal variation across the graph structure, helping to understand the way taxi pick-ups vary across streets, from corner to corner, possibly revealing important aspects of city dynamics. 
Within the universe of graph signal processing, we focus on spectral graph wavelets. The concept of graph wavelets is strongly related to the classical version on regular domains. However, some operations quite simple on that domain, such as the shift operation, are harder to define precisely on the graph scenario. Thus, the definition of the graph wavelets is based on the graph spectral theory, where, for instance, the shift operation can be interpreted as a multiplication. In the literature, there are two strands for the definition of the spectral domain for a graph, one based on the adjacency matrix (SANDRYHAILA; MOURA, 2013b) and other based on the graph Laplacian (HAMMOND; VANDERGHEYNST; GRIBONVAL, 2011). The spectral graph wavelet theory is more consolidated in the case of the graph Laplacian, so we follow it in this dissertation. Additionally, many interesting properties of a graph can be inferred from its spectral decomposition.

Nowadays, the construction of graph wavelets has been attracting the attention of several researchers around the world. Published in 2011, the initial proposal of spectral graph wavelets by Hammond et al. (HAMMOND; VANDERGHEYNST; GRIBONVAL, 2011) led to interesting variations in the last five years, such as spectral graph wavelets specialized in community mining (TREMBLAY; BORGNAT, 2014), groups of nodes well-connected together on a graph, and spectral graph wavelets adjustable to the spectrum of the graph (SHUMAN et al., 2015), which enable scalable vertex-frequency analysis (extension of classical time-frequency analysis to graph domains).

On the other hand, the use of such tools is still incipient with few applications such as community mining (TREMBLAY; BORGNAT, 2014), urban data visual analysis (VALDIVIA et al., 2015), and traffic event detection on transportation networks (MOHAN et al., 2014). In this sense, we contribute to the visual analysis of dynamic networks. The spectral graph wavelets are in the background of an interface that helps users to gain knowledge about behavior of elements forming a dynamic network. We resort to visual analytics (KEIM et al., 2008) because it allows us to combine the human's ability to understand patterns with the computer's capacity to process large amounts of data.

\subsection{Contributions of the Dissertation}

The main contributions of the dissertation are:

- A novel method for visual analysis of dynamic networks, and

- The appreciation of remarkable properties of the graph wavelets.

Specifically, the novel method for visual analysis of dynamic networks overcomes current state-of-the-art techniques bridging the gap between exploring the dynamic network as a whole and specific network elements, reducing the amount of direct inspections required to find 
relevant information. Thus, a user can acquire more knowledge about a dynamic network and its elements with less mental effort. Moreover, our visual analysis approach relies on a recently developed approximation mechanism (HAMMOND; VANDERGHEYNST; GRIBONVAL, 2011) to approximate the wavelet coefficients, reducing computational costs to enable the analysis of large dynamic networks. For instance, in one of our usage scenarios in Chapter 5, we processed a dynamic network with about 300 thousand nodes and 5 millions edges in just a few seconds, using a regular i7 computer.

In summary, our visual analytics method is able to represent the temporal evolution of a dynamic network in a simple visualization, which also acts as a visual index, guiding users directly to relevant time slices for further exploration and revealing interaction patterns, regardless of the involved elements and their temporal distance.

The appreciation of remarkable properties of the graph wavelet transform is made providing examples of how the choice of the graph wavelet transform, signal under analysis, and graph topology impact the computation of the wavelet coefficients. Our goal is not to provide a rigorous treatment towards understanding their effect on the wavelet coefficients, but to shed some light on it to help inexperienced researchers in the use of these tools. In fact, the complete understanding of the synergy among the graph wavelet transform, signal under analysis, graph topology, and wavelet coefficients is still a open question.

In summary, we bring some intuition from the classical signal processing theory to the more complex scenario of graph domains relying on illustrative examples (a set of controlled experiments using synthetic and real-world data).

\subsection{Publications Directly Related to the Dissertation}

The items below list the publications directly related to the dissertation, which were originated during the development of the doctoral project:

- DAL COL, A.; VALDIVIA, P.; PETRONETTO, F.; DIAS, F.; SILVA, C. T.; NONATO, L. G. Wavelet-based visual analysis for data exploration. Computing in Science \& Engineering, Institute of Electrical and Electronics Engineers, v. 19, n. 5, p. 85-91, 2017.

- __ Wavelet-based visual analysis of dynamic networks. IEEE Transactions on Visualization and Computer Graphics, Institute of Electrical and Electronics Engineers, 2017.

The article "Wavelet-based visual analysis for data exploration" was published in Computing in Science \& Engineering (CiSE). The magazine is commonly read by readers from a variety of computational fields and specialists from various disciplines, such as physics, medicine, and astronomy, thus enabling wide dissemination of the properties of the graph wavelets. The 
article "Wavelet-based visual analysis of dynamic networks" was accepted by IEEE Transactions on Visualization and Computer Graphics (TVCG), the most relevant journal in the field of visualization. Table 1 depicts important journal citation metrics according to IEEE Xplore $<$ http://ieeexplore.ieee.org $>$. These information highlight the relevance of the selected journals.

Table 1 - Journal citation metrics according to IEEE Xplore. Update at March 14, 2018.

\begin{tabular}{c|c|c|c}
\hline Avenue & Impact Factor & Eigenfactor & Article Influence Score \\
\hline \hline TVCG & 2.840 & 0.01808 & 1.156 \\
\hline CiSE & 2.074 & 0.00474 & 1.309 \\
\hline
\end{tabular}

\subsection{Dissertation Outline}

The dissertation is organized in six chapters and one appendix as follows:

Chapter 1 [Introduction]: introduces the dissertation by positioning it according to the existing literature, it goes from the more general scenario of graph signal processing to the more specific scenario of graph wavelet analysis, where our work fits.

Chapter 2 [Review of the Literature]: reviews the literature focusing mainly on graph signal processing theory, spectral graph wavelets, and methods for visual analysis of dynamic networks.

Chapter 3 [Spectral Graph Theory]: describes the well-established theoretical and computational aspects of spectral graph theory.

Chapter 4 [Illustrative Interpretation of Graph Wavelets]: presents a generalization of the spectral graph wavelets, the spectrum-adapted (tight) graph wavelets, and explores differences between both spectral graph wavelet transforms. Furthermore, some remarkable properties of the graph wavelets are presented through illustrative examples, which use synthetic and real-world data.

Chapter 5 [Visual Analysis of Dynamic Networks]: introduces a novel method for the visual analysis of dynamic networks, compares the new method against state-of-the-art techniques, and discusses some limitations of the method. In addition, two real-world dynamic networks are investigated.

Chapter 6 [Conclusion]: stresses the importance of the dissertation and presents directions for future work. 
Appendix A [Demonstrations]: details proofs and developments of important formulas that have been omitted in the original publications. 

CHAPTER

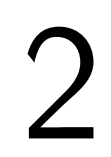

2

\section{REVIEW OF THE LITERATURE}

The review of the literature is organized as two separate sections, graph signal processing $\&$ graph wavelets and visual analytics of dynamic networks.

\subsection{Graph Signal Processing \& Graph Wavelets}

Signal processing theory in graph domains encompasses a number of different approaches, such as node graph wavelets (CROVELLA; KOLACZYK, 2003), spectral graph wavelets (HAMMOND; VANDERGHEYNST; GRIBONVAL, 2011), spectrum-adapted (tight) graph wavelets (SHUMAN et al., 2015), windowed graph Fourier transform (SHUMAN; RICAUD; VANDERGHEYNST, 2016), just to name a few. Shuman et al. (SHUMAN et al., 2013) survey graph signal processing theory and indicate the different ways one can define graph spectral domains from the so-called non-normalized graph Laplacian and normalized graph Laplacian. Sandryhaila and Moura (SANDRYHAILA; MOURA, 2013b) introduce the adjacency matrix as an alternative option to define graph spectral domains. Adjacency matrices allow the analysis of directed graphs, however they may not be diagonalizable, requiring the use of Jordan decomposition. Additionally, this strand does not have yet a wavelet theory associated to it.

Applications of graph signal processing include anomalous subgraph detection (MILLER et al., 2015), 3D point cloud compression (ZHANG; FLORÊNCIO; LOOP, 2014), graph Fourier transform centrality (SINGH; CHAKRABORTY; MANOJ, 2017), semi-supervised learning (BELKIN; MATVEEVA; NIYOGI, 2004; SANDRYHAILA; MOURA, 2013a; ZHOU; SCHÖLKOPF, 2004; ZHOU et al., 2004), and vertex-frequency analysis (SHUMAN et al., 2015; SHUMAN; RICAUD; VANDERGHEYNST, 2016).

A toolbox for signal processing on graphs, called Graph Signal Processing Toolbox (GSPBOX), is currently available in the Matlab and Python programming languages (PERRAUDIN et al., 2014). The toolbox implements graphs, plots, operators, filters, Chebyshev polynomial 
approximation to speed up filtering (HAMMOND; VANDERGHEYNST; GRIBONVAL, 2011; SHUMAN; VANDERGHEYNST; FROSSARD, 2011), among other functionalities. Susnjara et al. (SUSNJARA et al., 2015) recently proposed a Lanczos-based approximation that reaches at least the same accuracy as the Chebyshev polynomial approximation without significantly increasing the overall computational complexity.

Crovella and Kolaczyk (CROVELLA; KOLACZYK, 2003) design node graph wavelets to study byte stream networks (networks where bytes flow over links). These graph wavelets are defined directly on the node domain using the concept of $h$-hop neighborhood (set of nodes that are less than or equal to h hops from a node). The main idea behind these graph wavelets is to compare the signal within a given region (disk) with the signal in a surrounding region (ring) both centered in a node and adapted to a scale.

Spectral graph wavelets have been receiving special attention (HAMMOND; VANDERGHEYNST; GRIBONVAL, 2011), because they permit the interpretation of spectral properties as in Fourier analysis, while enabling local analyses of a graph. Applications include visual analysis of time-varying data defined on graph nodes (VALDIVIA et al., 2015), community mining (TREMBLAY; BORGNAT, 2014), and traffic event detection on transportation networks (MOHAN et al., 2014).

Graph wavelets are particularly useful to detect local variations of a signal defined on a graph, which are reflected in the wavelet coefficients. Such fact can be used to direct users to patterns on graphs. Valdivia et al. (VALDIVIA et al., 2015) consider the wavelet coefficients of the graph wavelets to enable the visual analysis of urban data, allowing the identification of patterns from a limited amount of information (Figure 1a). Valdivia's formulation is only able to handle static networks where information associated with nodes varies over time. The topology of the network remains unchanged because Cartesian graphs (SANDRYHAILA; MOURA, 2014) are considered to allow the use of mathematical properties that reduce computational cost. Even restricted to static networks the computational effort is still significant and large networks can not be processed in a reasonable time. In addition, Valdivia's visual metaphor employees stacked graphics to support the visualization of different frequency classes. Although quite informative, these graphics are not appropriated as the frequency classes are not balanced.

Tremblay and Borgnat (TREMBLAY; BORGNAT, 2014) employ graph wavelets for multiscale detection of communities (groups of nodes well-connected together) (Figure 1b). When dealing with dynamic networks, the whole data is aggregated in a preprocessing step, not considering the temporal evolution of information. Additionally, the grouping is performed on each wavelet scale individually.

Mohan et al. (MOHAN et al., 2014) analyze the wavelet coefficients of the graph wavelets to infer occurrence, spread, propagation, and span of traffic congestion (Figure 1c). Road segments are considered as graph nodes, which are connected if a vehicle can go from one segment to another, and the average speed of all vehicles passing through a road segment is 
the signal. Mohan et al. advance the study of transportation networks considering appropriately spatial relations between different road segments, which were typically modeled as time series.

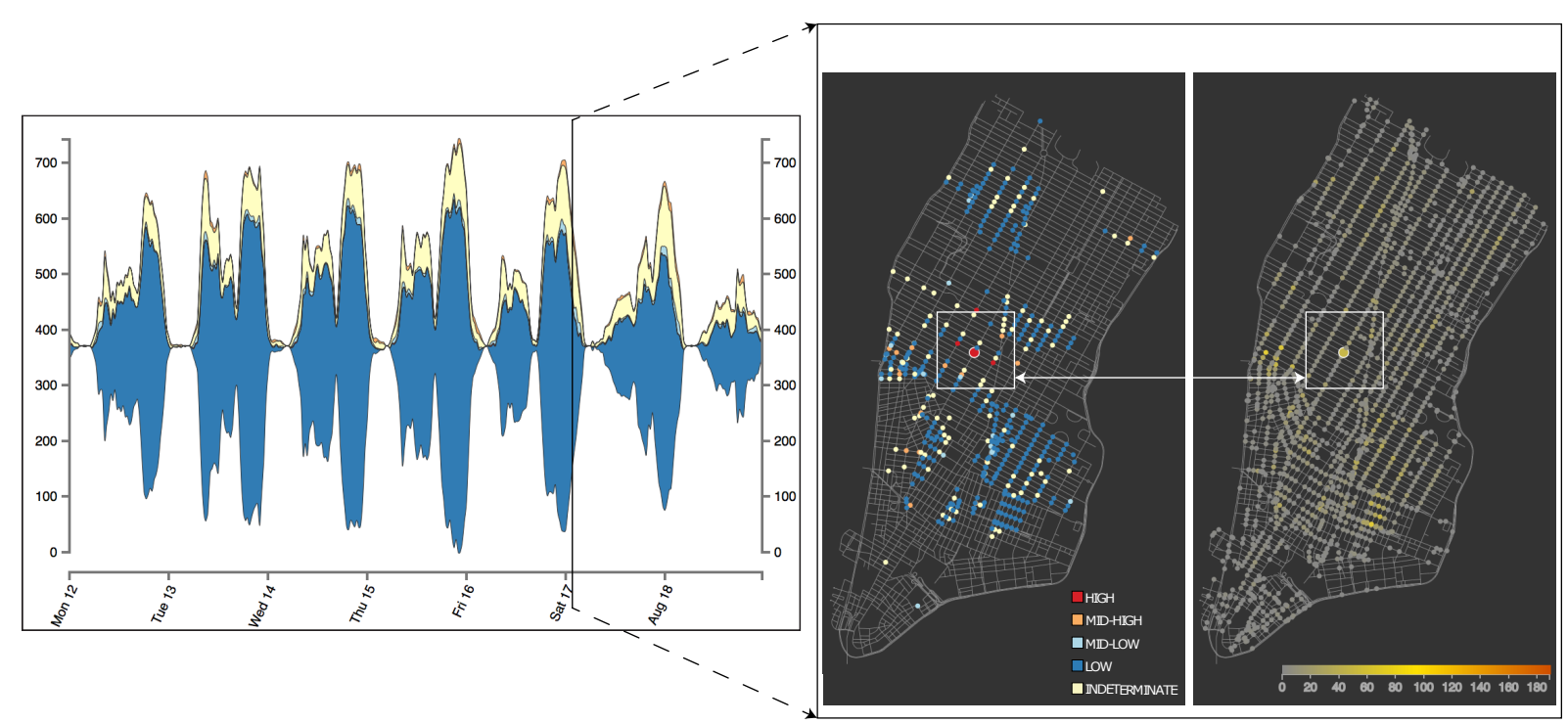

(a) Visual analysis of urban data, which involves taxi pick-ups in downtown Manhattan (VALDIVIA et al., 2015)

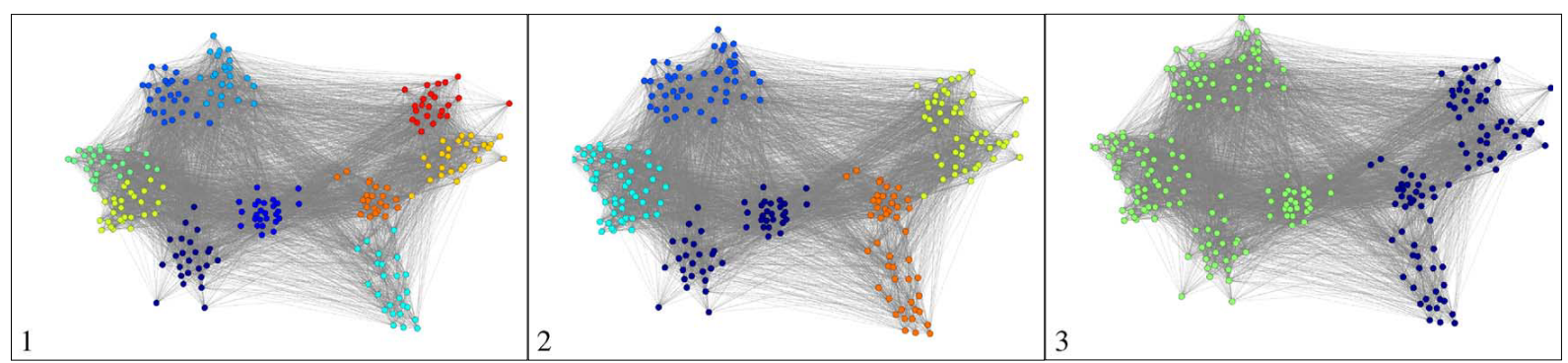

(b) Multiscale detection of communities (TREMBLAY; BORGNAT, 2014)
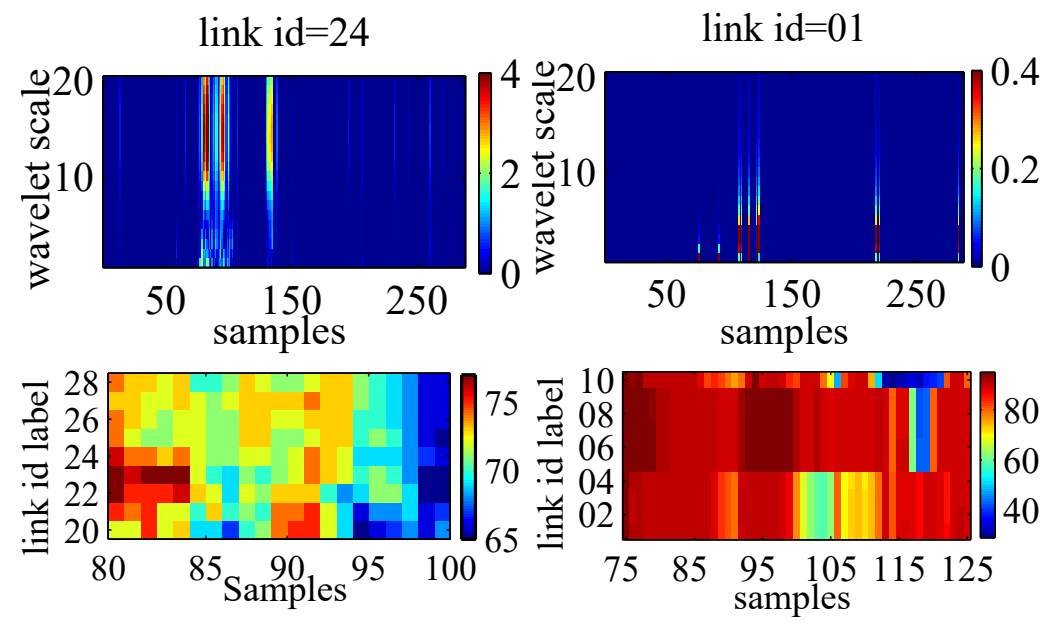

(c) Exploration of transportation networks (MOHAN et al., 2014)

Figure 1 - Graph wavelet data analysis.

The approach we proposed in this dissertation, in contrast to Tremblay, explores the temporal evolution of dynamic networks and builds node feature vectors that encompass all scales 
simultaneously. Furthermore, contrary to Valdivia, we use time series emphasizing dominant frequencies in our visual metaphor, since frequency classes are not balanced in our context.

We exploit the interesting properties of the graph wavelets and rely on a mechanism with low computational cost, thus allowing the analysis of large graphs. While other methods are capable of highlighting specific changes on graphs, the sensitivity of graph wavelets to signal variation has not been properly explored, a gap we bridge in this dissertation.

\subsection{Visual Analytics of Dynamic Networks}

The literature about dynamic network visualization is extensive (ARCHAMBAULT et al., 2014; BECK et al., 2014; BECK et al., 2016; HADLAK; SCHUMANN; SCHULZ, 2015; VEHLOW; BECK; WEISKOPF, 2015), with the vast majority of methods focusing on different manners of visually representing the network, mainly accounting for temporal variations. These methods can be roughly grouped into animation and timeline-based techniques.

Animation techniques represent dynamic networks through a sequence of frames. Networks are usually represented with node-link diagrams, with a stable layout in order to preserve context. Online methods are characterized by the use only of the previous time slices to build the layout in a given time slice. On the other hand, offline methods are those methods that employ all time slices in the sequence to define the layout in a specific time slice (DIEHL; GÖRG, 2002).

Tracking temporal changes is facilitated by transitions, such as highlighting differences on the network. Bach et al. (BACH; PIETRIGA; FEKETE, 2014) use blue halos to indicate nodes and edges being added and red halos to nodes and edges being removed. A time cursor allows transitions to be explored effectively (Figure 2a). Friedrich and Eades (FRIEDRICH; EADES, 2002) proposed an animation process that applies rigid movements to a graph, leading it from a initial position to a destiny as close as possible to the final position. Next, nodes are repositioned through a linear interpolation among intermediate and final positions (Figure 2b). Erten et al. (ERTEN et al., 2004) evidence differences between two consecutive time slices by means of difference graphs (Figure 2c).

While changes in adjacent times are quite evident, animation-based methods demand greater cognitive effort and time to track far apart changes in the network, mainly when many time slices are involved.

Timeline-based techniques are static representations that map time into space, illustrating the evolution of a network as an image. Some methods are able to change the information associated with each node in each time (BURCH et al., 2011; DANG; PENDAR; FORBES, 2016; ELZEN et al., 2014), visually representing the information through glyphs (FUCHS et al., 2013; SARAIYA; LEE; NORTH, 2005) or matrices (STEIN; WEGENER; SCHLIEDER, 2010; VEHLOW; BECK; WEISKOPF, 2016; YI; ELMQVIST; LEE, 2010). 

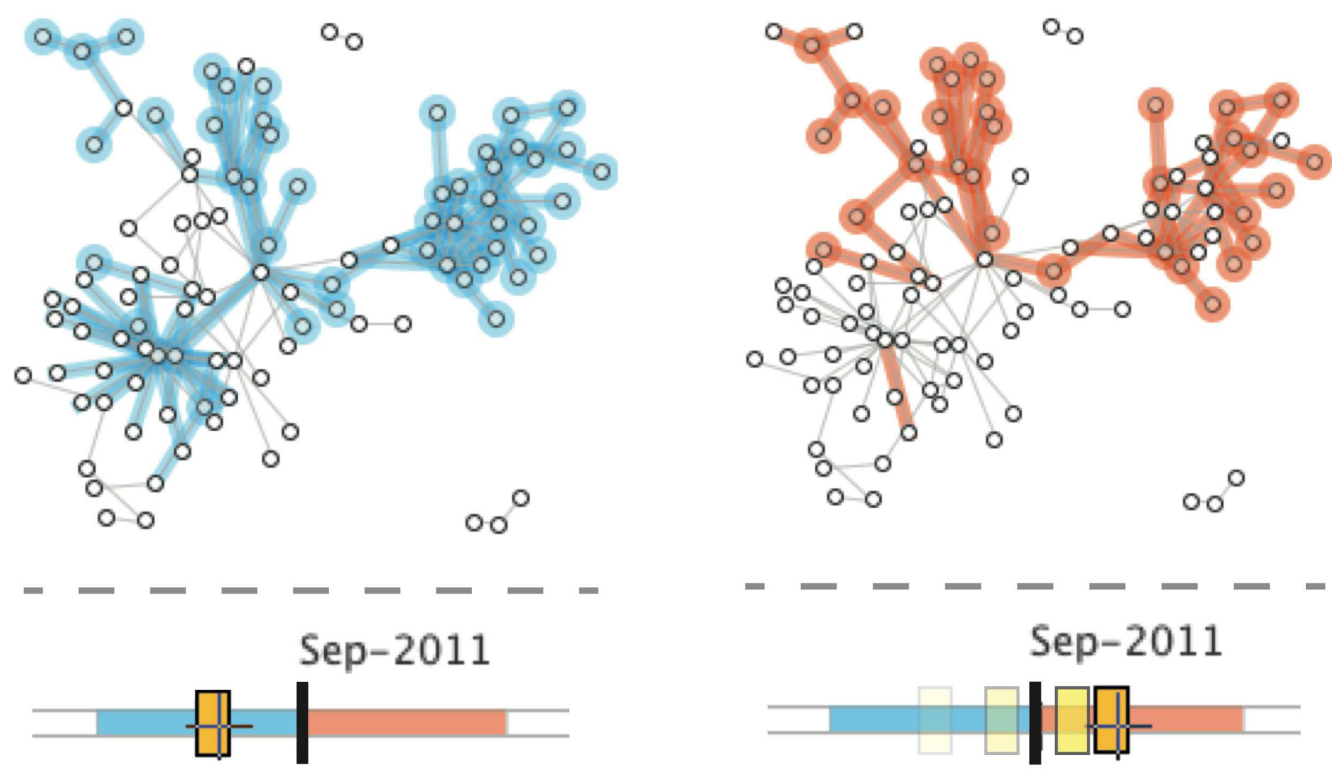

(a) GraphDiaries (BACH; PIETRIGA; FEKETE, 2014)

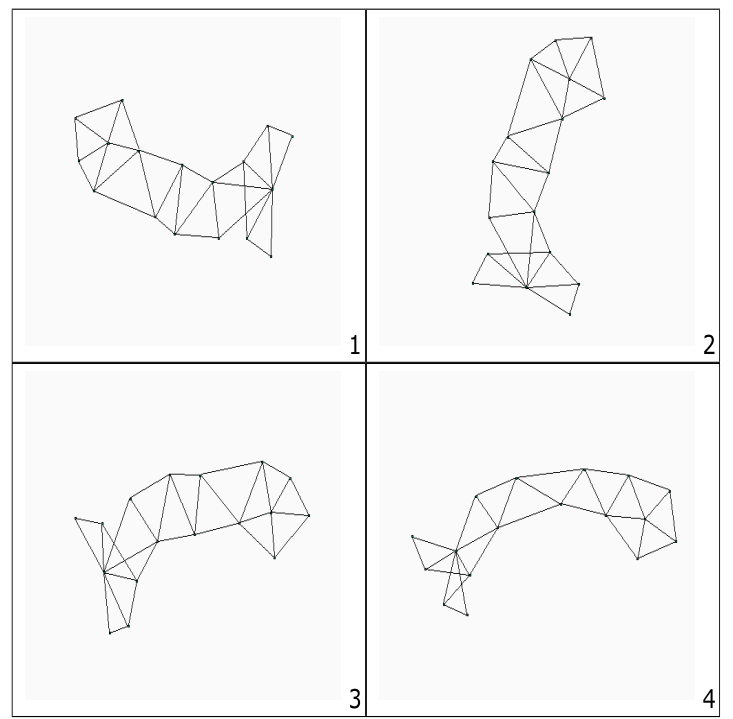

(b) Friedrich and Eades' method (FRIEDRICH; EADES, 2002)

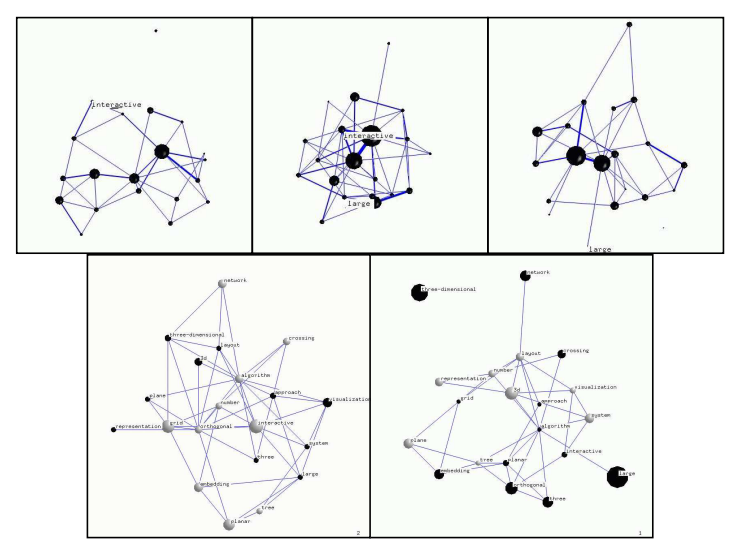

(c) GraphAEL (ERTEN et al., 2004)

Figure 2 - Animation techniques.

Saraiya et al. (SARAIYA; LEE; NORTH, 2005) conducted a qualitative analysis of glyphs as visualization technique for dynamic networks. The study reveals that the number of attributes shown in the nodes influences the accuracy of the analysis, and the number of views impacts the performance time. Additionally, in a controlled experiment, Fuchs et al. (FUCHS et al., 2013) compare four different types of glyphs (lines, stripes, clocks, and stars) while representing temporal properties visually.

Small multiples depicting different temporal moments have also been used as visual resource (FARRUGIA; HURLEY; QUIGLEY, 2011; GOVE et al., 2011; SARAIYA; LEE; NORTH, 2005). Generally, each multiple reveals the state of the network in a time slice. Far- 
rugia et al. (FARRUGIA; HURLEY; QUIGLEY, 2011) propose an approach to detect node connectivity changes, however it does not generalize to the analysis of the network as a whole. Small multiples suffer from the problem of exchanging between the size and number of images, being difficult to determine the number of multiples to be used.

Visual representation of interactions between entities is also possible with timeline-based methods. Dang et al. (DANG; PENDAR; FORBES, 2016) use arcs to connect related entities (Figure 3a). Dang's method allows the identification of activity patterns between entities along the time. Additionally, several interactive features enable the inspection of a particular group of entities or a relationship of interest. Elzen et al. (ELZEN et al., 2014) propose an extension of Massive Sequence Views (MSV), the MSV circular, to represent dynamic networks (Figure 3b). Arcs and radius encode interaction between elements and time, respectively. Structural aspects of a dynamic network, such as communities and stars, and temporal characteristic, such as trend, periodicity, and anomaly can be found by the circular MSV. Moreover, it is possible to observe correlations between communication behavior and information associated with the nodes, health status, for instance.

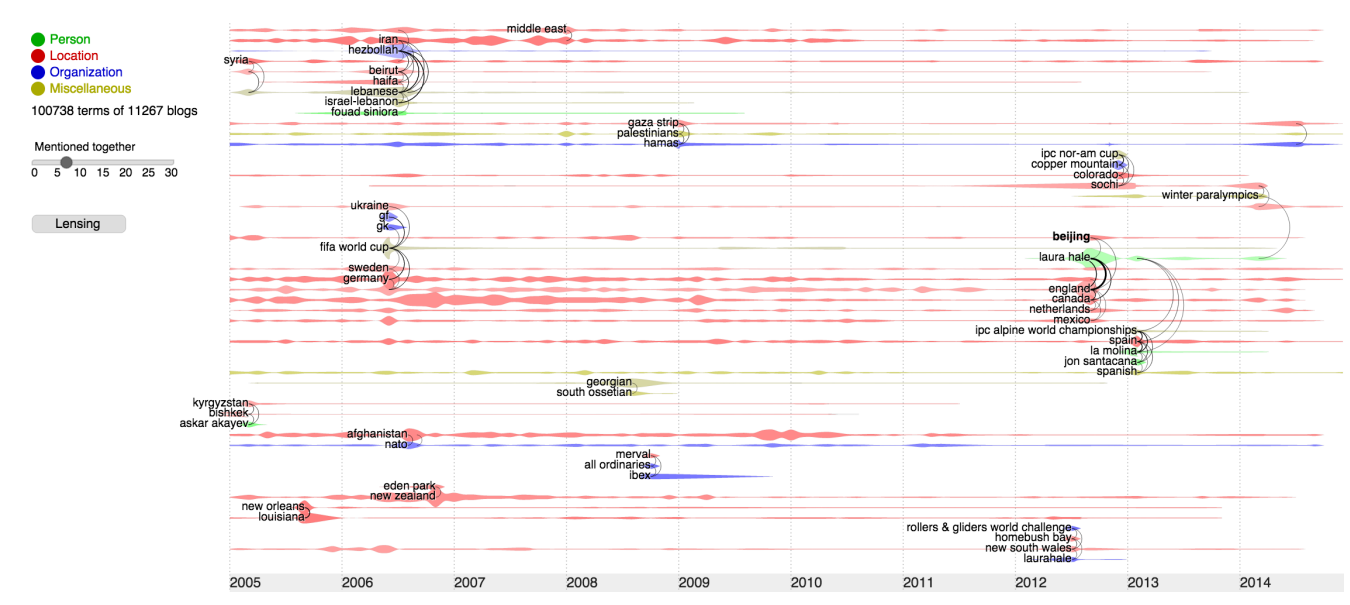

(a) TimeArcs (DANG; PENDAR; FORBES, 2016)

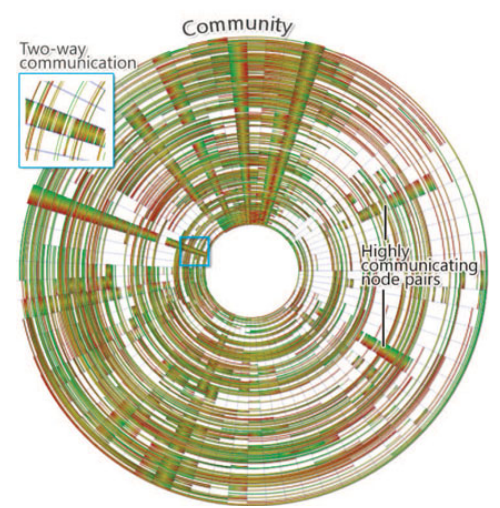

(b) MSV circular (ELZEN et al., 2014)

Figure 3 - Timeline-based techniques. 
Matrices have been used both to represent a single time slice and to summarize the entire dynamic network. In the first case, usually small multiples help in the understanding of the whole. MultiPiles (BACH et al., 2015) uses adjacency matrices to represent time slices (Figure 4a). The system piles the adjacency matrices interactively or automatically, so that each pile represents a state of the network. Piles are able to cover irregular time periods, which can still be explored to detect sudden changes. MultiPiles is able to explore long periods of time, but a large amount of elements in the network leads to large cover matrices (matrices displayed over the piles), increasing the visual complexity. Stein et al. (STEIN; WEGENER; SCHLIEDER, 2010) summarize the dynamics of the dynamic network as a single adjacency matrix (Figure $4 b$ ). In the adjacency matrix, each line and column corresponds to a node, where the entries indicate the weight of the edge connecting the corresponding nodes. Actually, the entire timeline is placed in an entry of the adjacency matrix using a glyph, which displays the timeline folded line by line. Although matrix-based representations are less attractive to general users, they avoid communicational disorder problems (GHONIEM; FEKETE; CASTAGLIOLA, 2004).

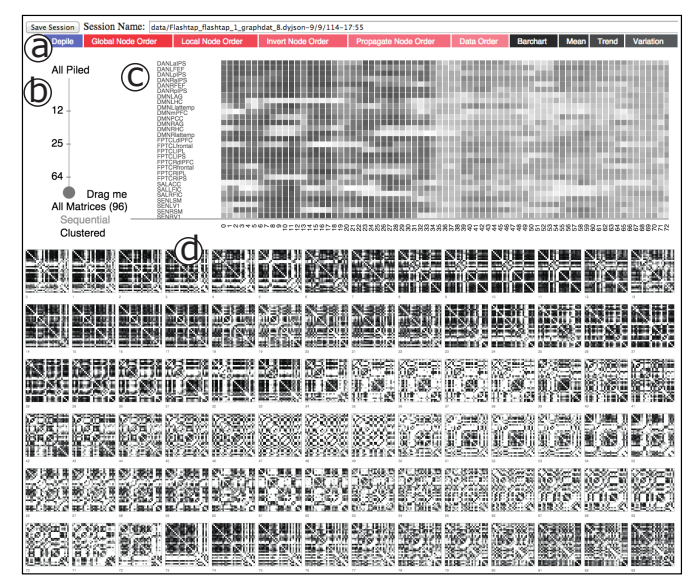

(a) MultiPiles (BACH et al., 2015)

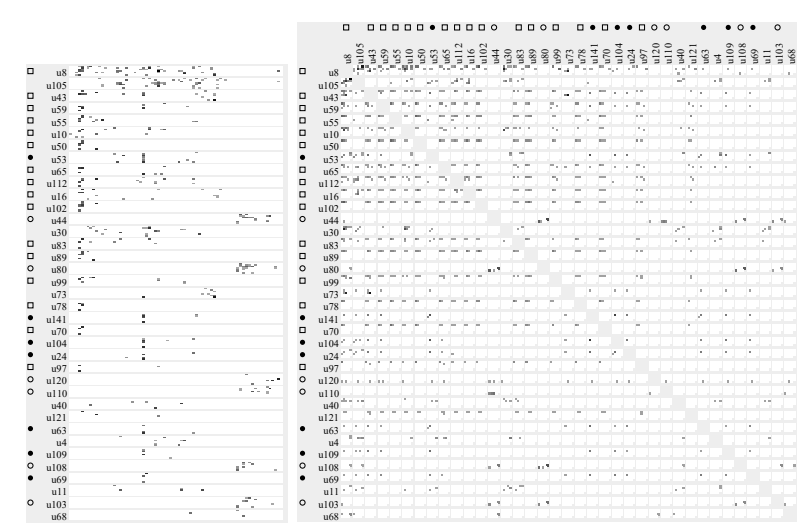

(b) PONV (STEIN; WEGENER; SCHLIEDER, 2010)

Figure 4 - Matrix-based techniques.

Scalability is an issue for timeline-based techniques. Alternatives to mitigate the problem have been proposed, as for example the aggregation of elements, but at the expense of hiding relevant information. Hadlak et al. (HADLAK et al., 2013) group nodes and edges of a dynamic network to attenuate scalability problems (Figure 5). The grouping process takes into account the time series associated with nodes and edges to create a supergraph. Although the supergraph provides an overview of the network, local changes are hidden.

Recent efforts try to reduce the shortcomings of both representations by considering more information from the network. Cui et al. (CUI et al., 2014) rely on static flow visualization to show changes in particular properties of the nodes (Figure 6), e.g. degree and centrality. Their method is able to reveal interesting patterns in the data, however, finding repetitive patterns is not easy and the relations between nodes are also not properly exploited. 


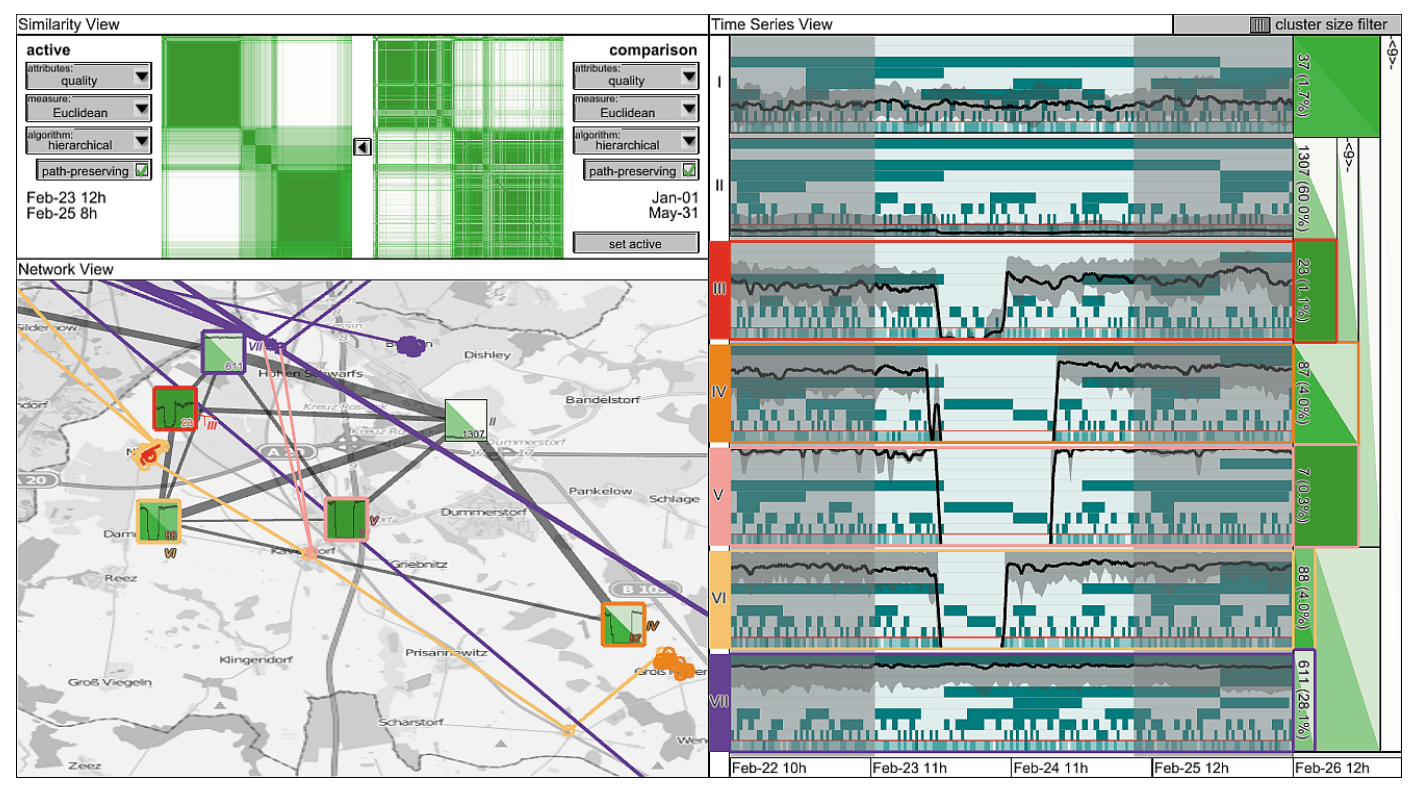

Figure 5 - Hadlak's method (HADLAK et al., 2013).

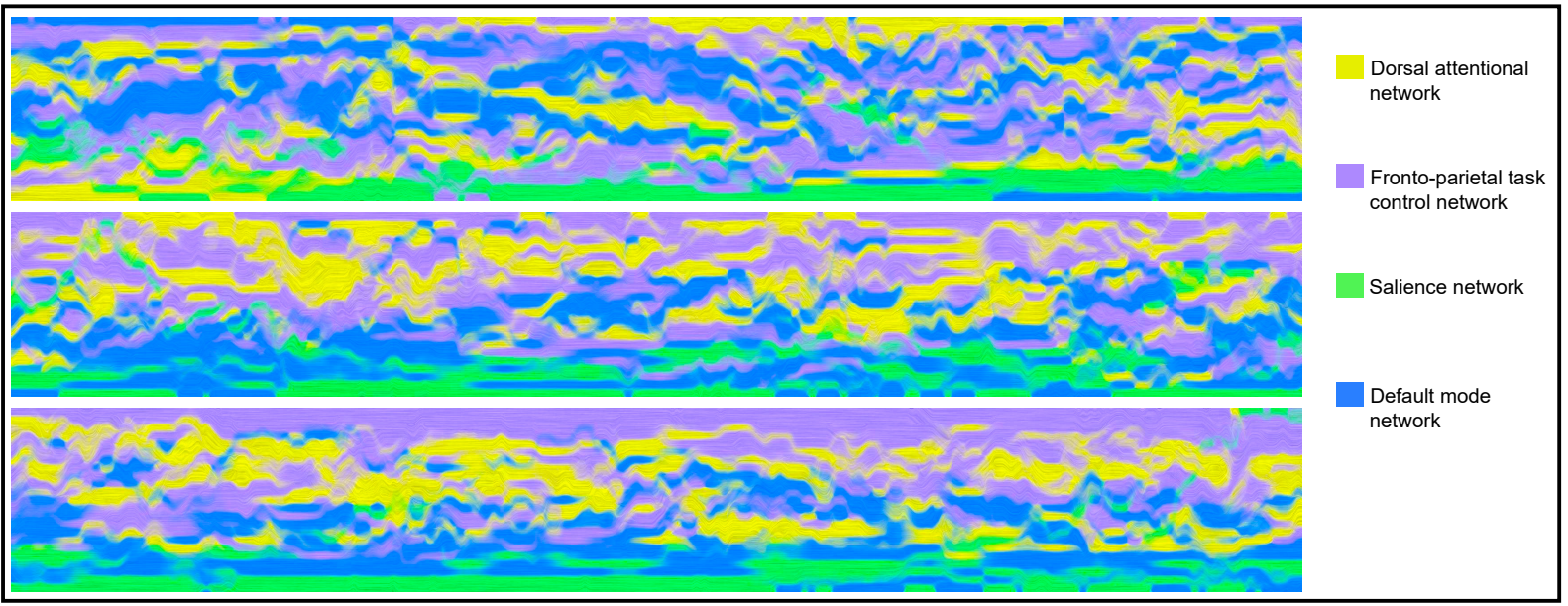

Figure 6 - GraphFlow (CUI et al., 2014).

Projections have been used to convey information about the structure of dynamic networks (BACH et al., 2016; CAO et al., 2016; STEIGER et al., 2014; ELZEN et al., 2016; LANDESBERGER et al., 2015), considering mainly Multidimensional Scaling (MDS). Those methods can be used to project feature vectors that characterize the network structure, or other information of interest, and the resulting projection can be used to identify clusters and anomalies.

Elzen et al. (ELZEN et al., 2016) represent states of a dynamic network as adjacency matrices and transform these adjacency matrices into vectors through a vectorization process. The resulting vectors are projected on a two-dimensional space to be explored (Figure 7a). Stable states, recurrent states, anomalous topologies can be visually detected. In addition, one can obtain knowledge about the transition between states. Bach et al. (BACH et al., 2016) introduce the Time Curves to visualize the temporal evolution of more general data (Figure $7 \mathrm{~b}$ ). The Time 
Curves is applicable in any data domain where it is possible to define a measure of distance between time slices, somehow generalizing the Elzen's method. Bach's method is able to reveal many patterns, such as regular progressions, abrupt changes, reversal for previous states. The performance and effectiveness of these methods are dependent on the definition of the feature vectors.

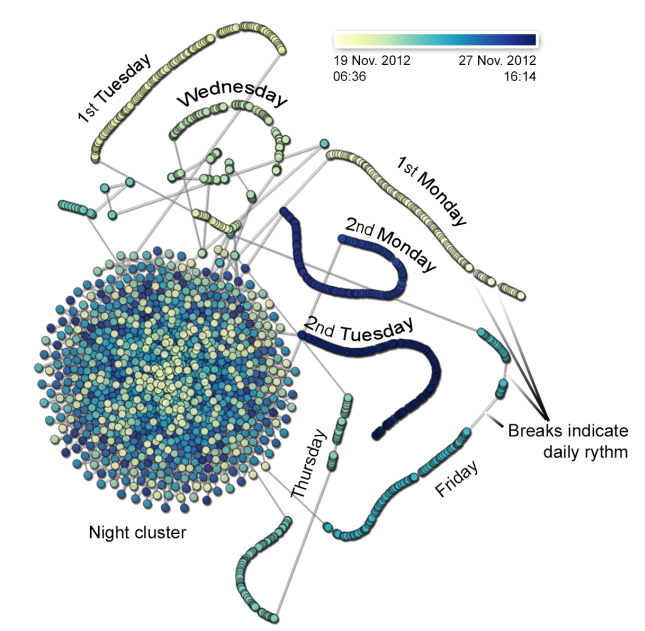

(a) Reducing Snapshots to Points (ELZEN et al., 2016)

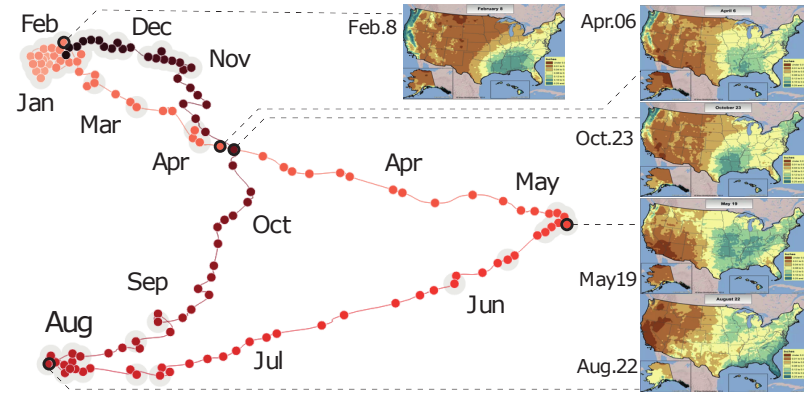

(b) Time Curves (BACH et al., 2016)

Figure 7 - Projection as support for dynamic network exploration.

Presented in Chapter 5, our method for visual analytics of dynamic networks allows the automatic detection of local variations, establishing a relationship between each element and its contact partners. In contrast, animation and timeline-based techniques that enable similar investigation require the user to mentally compare objects and their neighbors, tending to demand more cognitive effort. 

CHAPTER

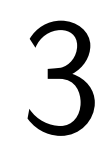

\section{SPECTRAL GRAPH THEORY}

Spectral graph theory refers to the analysis of graphs by means of linear algebra concepts. Important references in the literature study the spectrum of a graph and its applications (CHUNG, 1997; SPIELMAN, 2007). In this chapter, we review the spectral graph theory and spectral graph wavelet transform. The spectral graph wavelet transform is derived from the graph Fourier transform, which extends the idea of Fourier modes and frequencies to graph domains.

\subsection{Basic Definitions}

A graph $G$ is a structure composed of a set of nodes and a set of edges connecting pairs of nodes. Consider a graph $G=(V, E)$ with a node set $V=\left\{\tau_{1}, \ldots, \tau_{n}\right\}$ and an edge set $E$, where $n$ is the number of nodes. A weight $w_{i j} \in \mathbb{R}_{+}$is associated to each pair of nodes $\tau_{i}$ and $\tau_{j}$ in $V$, encoding how strong the relationship between them is. Since we do not consider self-loops $w_{i i}=0, i=1, \ldots, n$. We set $w_{i j}=0$ if there is no edge connecting nodes $\tau_{i}$ and $\tau_{j}$, $i \neq j$. Assigning weights to the relations between nodes makes the graph $G$ a weighted graph.

The adjacency matrix of a graph $G$ is a $n \times n$ matrix $W=\left(w_{i j}\right)$ with entries $w_{i j}$. We assume that the relation between two nodes is always symmetric, then $w_{i j}=w_{j i}$ and the graph $G$ is called undirected graph. The graph Laplacian, or combinatorial graph Laplacian, is a matrix given by $L=D-W$, where $W$ is the adjacency matrix and $D=\left(d_{i j}\right)$ is the degree matrix, a diagonal matrix with entries $d_{i i}=\sum_{k} w_{i k}$ (sum of the weights of all edges incident to node $\left.\tau_{i}\right)$. Since we consider only undirected graphs, both adjacency matrix and graph Laplacian are symmetrical. Generally, the eigenvalues of the adjacency matrix and graph Laplacian do not match generating different graph transforms. Although the adjacency matrix also extends the Fourier theory for graphs, we focus on the graph Laplacian because we employ the graph wavelet transform by Hammond et al. (HAMMOND; VANDERGHEYNST; GRIBONVAL, 2011), which uses the graph Laplacian. In terms of stability, the calculation of the eigenvectors is more robust in the case of graph Laplacian (SANDRYHAILA; MOURA, 2014). 
The graph Laplacian $L$ is real, symmetric, and semi-positive definite, then $L$ has a complete set of orthonormal eigenvectors $u_{\ell}, \ell=1, \ldots, n$, with corresponding non-negative real eigenvalues $\lambda_{\ell}, \ell=1, \ldots, n$, which we assume ordered in non-decreasing order $0=\lambda_{1} \leq$ $\lambda_{2} \leq \cdots \leq \lambda_{n}$. The zero eigenvalue has algebraic multiplicity equal to the number of connected components of the graph $G$. If the graph $G$ is connected ( $G$ has just one component), then zero appears as a simple eigenvalue and we may write $0=\lambda_{1}<\lambda_{2} \leq \cdots \leq \lambda_{n}$.

The graph Laplacian has a variation called normalized graph Laplacian defined as

$$
\mathscr{L}=D^{-\frac{1}{2}} L D^{-\frac{1}{2}}
$$

For any graph $G$, the eigenvalues of the normalized graph Laplacian form a subset of the interval $[0,2]$. The choice of the graph Laplacian (non-normalized or normalized) is a open question and depend on the application at hand. We opted for the non-normalized graph Laplacian since it produced excellent results in the exploration of dynamic networks. Further information about the use of the normalized graph Laplacian can be found in (NARANG; ORTEGA, 2012).

Additional information can be integrated into the graph through a real-valued function $f: V \rightarrow \mathbb{R}$. The function $f$ assigns a real value $f(\tau)$ to each node $\tau \in G$. We term the function $f$ as signal, or graph signal. The set $f(V)=\{f(\tau) \mid \tau \in V\}$ can be interpreted as a vector in $\mathbb{R}^{n}$, where the $i$-th entry of the vector corresponds to the value of the function in node $\tau_{i}$. Figure 8 illustrates temperature measurements (signal) detected by weather sensors (graph nodes) distributed throughout the United States. The graph Laplacian eigenvectors $u_{\ell}: V \rightarrow \mathbb{R}$, $\ell=1, \ldots, n$ can also be seen as signals on $G$ since they are defined on the node set $V$. Furthermore, the graph Laplacian satisfies

$$
(L f)(i)=\sum_{j} w_{i j}\left[f\left(\tau_{i}\right)-f\left(\tau_{j}\right)\right]
$$

where $(L f)(i)$ corresponds to the $i$-th entry of the vector $L f$.

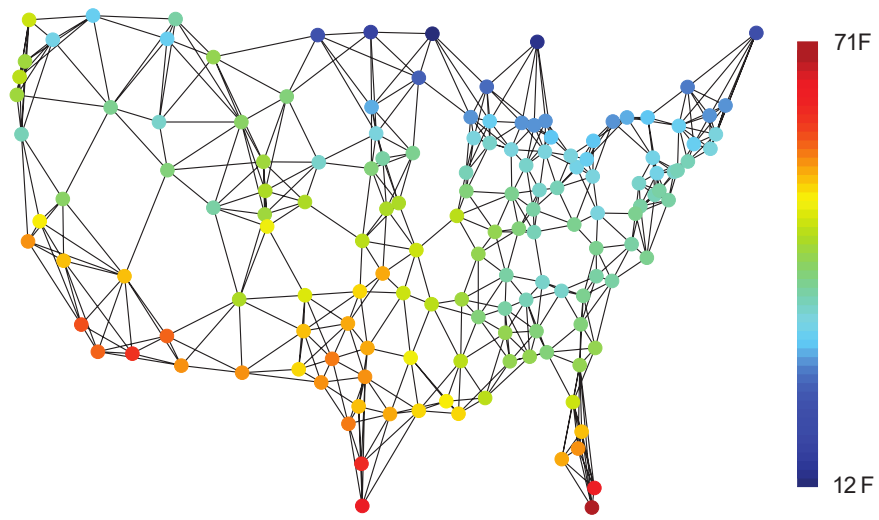

Figure 8 - Signal defined on the nodes of a graph. The signal encodes temperature measurements across the United States (SANDRYHAILA; MOURA, 2014). 
Let $f: V \rightarrow \mathbb{R}$ be a signal defined on a graph $G$, the set of zero crossings of $f$ on $G$ is

$$
Z_{G}(f)=\left\{\left(\tau_{i}, \tau_{j}\right) \in E \mid f\left(\tau_{i}\right) f\left(\tau_{j}\right)<0\right\}
$$

In other words, $Z_{G}(f)$ is the set of edges connecting nodes where $f$ has different signs (positive and negative). The larger the number of zero crossings $\left|Z_{G}(f)\right|$, the more $f$ changes its sign across the graph. Additionally, a path graph is a graph whose nodes are adjacent to exactly two other nodes, with the exception of the two extreme ones that are connected to only one node. Figure 9 depicts the number of zero crossings of the graph Laplacian eigenvectors $u_{\ell}$ for a path graph with 64 nodes. We assume the eigenvectors ordered in non-decreasing order of eigenvalues $\left(u_{1}, u_{2}, \ldots, u_{n}\right)$. The eigenvectors associated with larger eigenvalues oscillate more throughout the graph. Therefore, the eigenvalues and eigenvectors of the graph Laplacian can be interpreted as frequencies and Fourier modes similarly to the classical Fourier theory, that is, the larger the frequency (eigenvalue), the more oscillatory the corresponding Fourier mode (eigenvector).

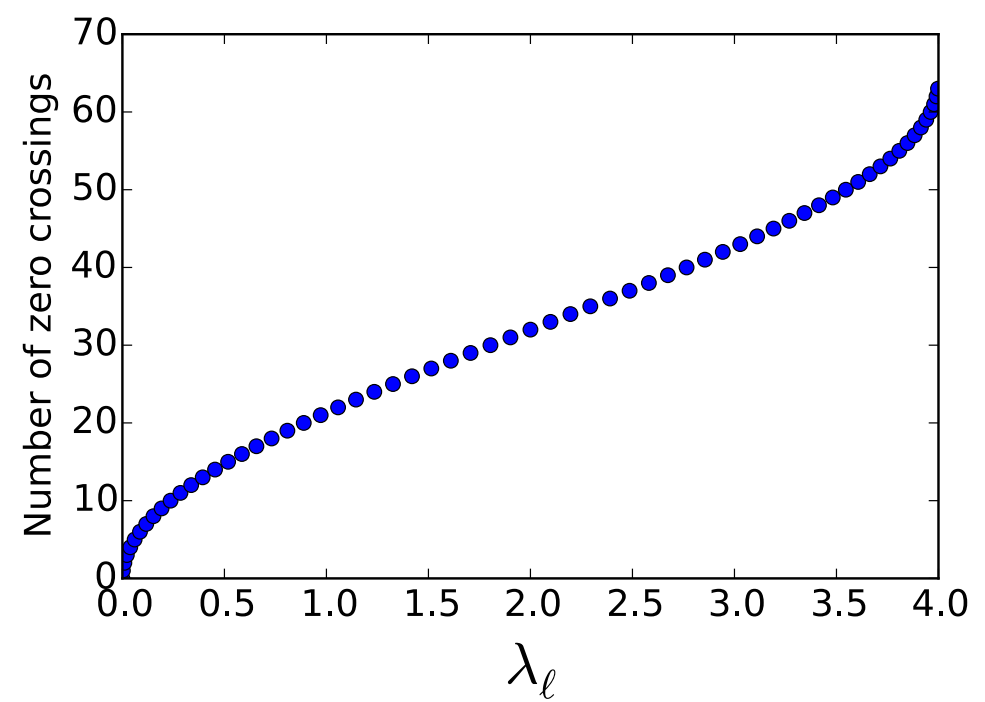

Figure 9 - Number of zero crossings of the graph Laplacian eigenvectors $\left(u_{1}, u_{2}, \ldots, u_{n}\right)$ for the path graph with 64 nodes.

\subsection{Graph Fourier Transform}

We initially define the classical Fourier transform on regular domains. The classical Fourier transform basically expands a function in terms of complex exponential functions $e^{i \lambda t}$, which are eigenfunctions of the classical Laplacian. In more mathematical terms, let $f: \mathbb{R} \rightarrow \mathbb{R}$ be a function defined on the regular domain $\mathbb{R}$, the Fourier transform of $f$ is a function $\hat{f}$ given by

$$
\hat{f}(\lambda)=\left\langle f, e^{i \lambda t}\right\rangle=\int_{\mathbb{R}} f(t) e^{-i \lambda t} d t
$$


The classical inverse Fourier transform consists of the integration of the basis functions $e^{i \lambda t}$ weighted by $\hat{f}(\lambda)$

$$
f(t)=\frac{1}{2 \pi} \int_{\mathbb{R}} \hat{f}(\lambda) e^{i \lambda t} d \lambda
$$

thus recovering the initial function $f$.

The graph Fourier transform and inverse graph Fourier transform are defined simply abstracting these concepts for graphs. The eigenfunctions of the classical Laplacian are replaced by the eigenvectors $u_{\ell}$ of the graph Laplacian. Thereby, given a signal $f: V \rightarrow \mathbb{R}$ defined on the nodes of $G$, the graph Fourier transform of $f$ is a function $\hat{f}$ defined by

$$
\hat{f}\left(\lambda_{\ell}\right)=\left\langle f, u_{\ell}\right\rangle=\sum_{i=1}^{n} f\left(\tau_{i}\right) u_{\ell}\left(\tau_{i}\right) .
$$

The inverse graph Fourier transform reconstructs the initial signal $f$ with the linear combination of the eigenvectors $u_{\ell}$ weighted by $\hat{f}\left(\lambda_{\ell}\right)$

$$
f=\sum_{\ell=1}^{n} \hat{f}\left(\lambda_{\ell}\right) u_{\ell}
$$

Similar to the classical Fourier transform, the graph Fourier transform reveals frequencies present in a signal $f$. The presence of high frequency Fourier coefficients $\left(\hat{f}\left(\lambda_{\ell}\right)\right.$ when $\lambda_{\ell}$ is in the rightmost part of the spectrum) indicates that a signal varies abruptly in some region(s) of the graph, while the presence of low frequency Fourier coefficients $\left(\hat{f}\left(\lambda_{\ell}\right)\right.$ when $\lambda_{\ell}$ is in the leftmost part of the spectrum) suggests smooth signal variation.

To illustrate the above concept and relate it to the classical Fourier transform, we consider again the path graph with 64 nodes. First, we use two eigenvectors as graph signals, one of low frequency and one of high frequency, more precisely $u_{11}$ and $u_{61}$. Figure 10 depicts both eigenvectors defined on the path graph (left and center signal panels). The graph Fourier transform of these signals is also depicted in Figure 10, corresponding to the spikes on the corresponding eigenvalues (frequencies), the same behavior as in the classical Fourier transform.

The graph Fourier transform provides a global description of signal behavior on the graph. Indeed, let $f_{0}$ be a signal defined as the combination of the low and high frequency eigenvectors discussed above. More precisely,

$$
f_{0}(\tau)=\left\{\begin{array}{ll}
u_{11}(\tau) & \text { if } \tau \in\left\{\tau_{i} \mid i=1, \ldots, 30\right\} \\
u_{61}(\tau) & \text { if } \tau \in\left\{\tau_{i} \mid i=31, \ldots, 64\right\}
\end{array} .\right.
$$

Figure 10 depicts signal $f_{0}$ on the right signal panel. Also shown in Figure 10, the graph Fourier transform of $f_{0}$ indicates the presence of low and high frequencies in the signal, suggesting that the signal has both smooth and abrupt variation. 


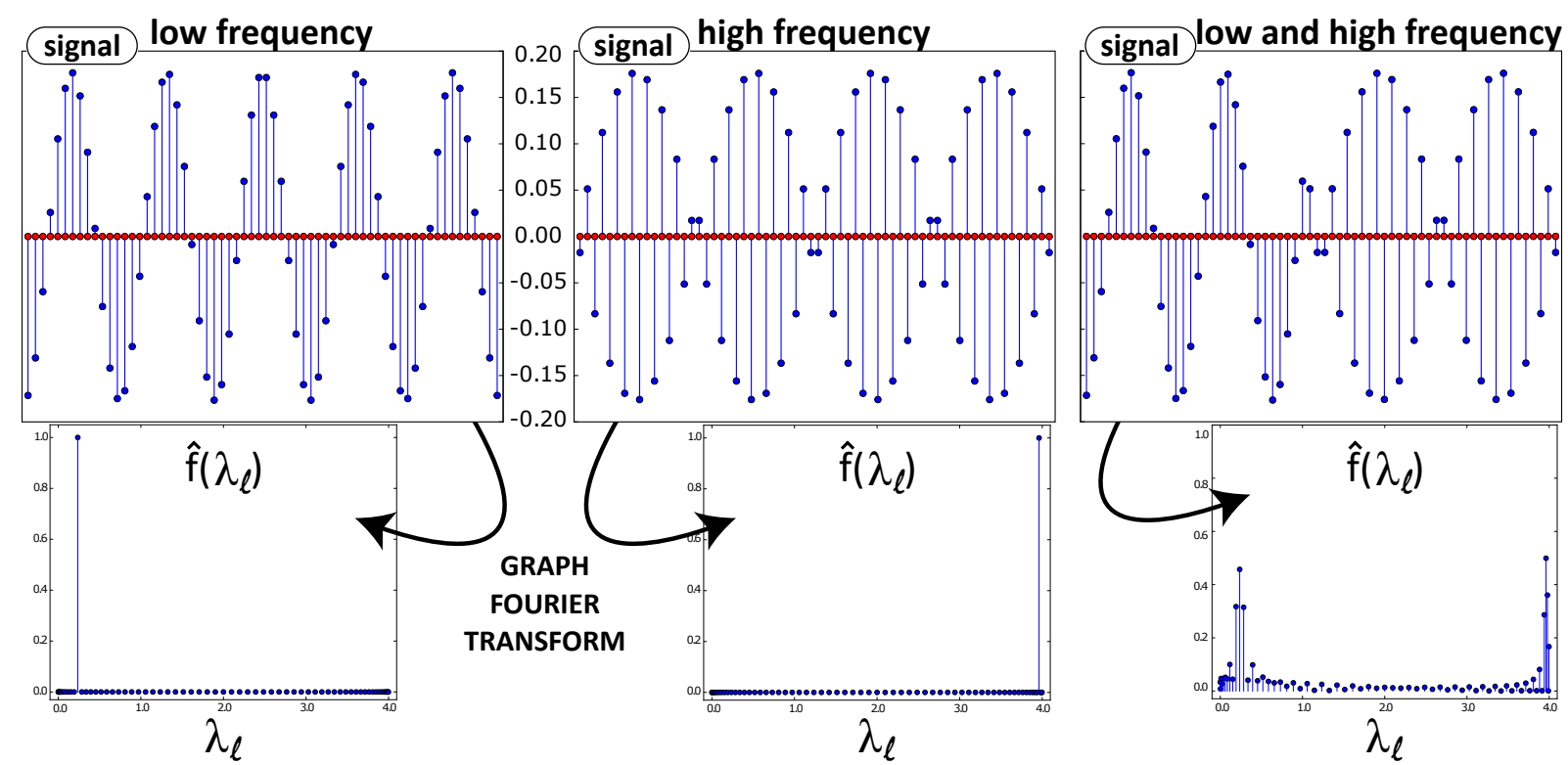

Figure 10 - Signals of low frequency (left), high frequency (center), and $f_{0}$ (right) defined on the path graph with 64 nodes. Signal $f_{0}$ is the combination of the low and high frequency signals. Red dots represent the graph nodes and the height of the vertical blue lines corresponds to the amplitude of the signal. The graph Fourier transforms are indicated by arrows. The graph Fourier transform corresponding to signal $f_{0}$ indicates the presence of low and high frequencies.

Other tools used in the classical signal processing, including convolution, translation, modulation, dilatation, and windowed graph Fourier transform, have generalizations into graph context. Such tools and more detailed information about them can be found in (SHUMAN et al., 2013; SHUMAN; RICAUD; VANDERGHEYNST, 2012).

\subsection{Spectral Graph Wavelet Transform}

We start again with classical concepts coming from regular domains. The classical wavelet transform is generated by a single wavelet $\psi$, called mother wavelet. The mother wavelet is used to create the classical wavelets $\psi_{s, a}: \mathbb{R} \rightarrow \mathbb{R}$ via translation and scaling

$$
\psi_{s, a}(t)=\frac{1}{s} \psi\left(\frac{t-a}{s}\right)
$$

where $s \in \mathbb{R}$ is a non-zero, positive scalar and $a \in \mathbb{R}$ is a scalar. An immediate extension of such equation to the graph context could be done as follows

$$
\psi_{s, l}(\tau)=\frac{1}{s} \psi\left(\frac{\tau-\imath}{s}\right)
$$

However, it is difficult to assign a meaning to the difference of two nodes $(\tau-\imath)$ and the product between a scalar and a node $\left(s^{-1}(\tau-\imath)\right.$ ). Hammond et al. (HAMMOND; VANDERGHEYNST; 
GRIBONVAL, 2011) proposed an alternative to circumvent this problem. As we shall see, their approach is based on the graph Fourier transform.

Motivation: Result of the classical wavelet transform, the classical wavelet coefficients are obtained by expanding a function $f: \mathbb{R} \rightarrow \mathbb{R}$ in terms of the classical wavelets

$$
W_{f}(s, a)=\left\langle f, \psi_{s, a}\right\rangle
$$

Using the inner product of two functions, we have

$$
W_{f}(s, a)=\int_{\mathbb{R}} f(t) \psi_{s, a}(t) d t=\int_{\mathbb{R}} f(t) \frac{1}{s} \psi\left(\frac{t-a}{s}\right) d t
$$

Let's define an auxiliary function $h_{s}: \mathbb{R} \rightarrow \mathbb{R}$ as $h_{s}(t)=\frac{1}{s} \psi\left(-\frac{t}{s}\right)$, then

$$
h_{s}(a-t)=\frac{1}{s} \psi\left(-\frac{a-t}{s}\right)=\frac{1}{s} \psi\left(\frac{t-a}{s}\right)
$$

thus combining Equations 3.12 and 3.13,

$$
W_{f}(s, a)=\int_{\mathbb{R}} f(t) h_{s}(a-t) d t=\left(f \star h_{s}\right)(a),
$$

where $\star$ indicates the convolution operator. The classical wavelet operator $T^{S}$ is a operator satisfying

$$
\begin{aligned}
T^{s} f: \mathbb{R} & \rightarrow \mathbb{R} \\
a & \rightarrow W_{f}(s, a)
\end{aligned}
$$

thus $T^{s} f(a)=W_{f}(s, a)=\left(f \star h_{s}\right)(a)$ (Equation 3.14). Applying the Fourier transform and using the convolution theorem

$$
\widehat{T^{s} f}(\lambda)=\widehat{f \star h_{s}}(\lambda)=\hat{f}(\lambda) \widehat{h_{s}}(\lambda)
$$

Using the definition of $h_{s}$ and the scaling property, we obtain

$$
\widehat{h_{s}}(\lambda)=\frac{1}{s} s \widehat{\psi}(-s \lambda)=\widehat{\psi}(-s \lambda)
$$

and finally

$$
\widehat{T^{s} f}(\lambda)=\hat{f}(\lambda) \widehat{\psi}(-s \lambda)
$$


Thereby, in the Fourier domain, the classical wavelet operator $T^{s}$ applied to a function $f$ can be obtained by the product between the Fourier transform of $f$ and a kernel $\widehat{\psi}$ scaled by $s$.

Generalization for graphs: The graph wavelet operator $T^{s}$ is defined so that when applied to a function $f, T^{s} f: V \rightarrow \mathbb{R}$, the graph Fourier transform is given by the multiplication between the graph Fourier transform of $f$ and a kernel $\hat{g}$ scaled by $s$

$$
\widehat{T^{s} f}\left(\lambda_{\ell}\right)=\hat{f}\left(\lambda_{\ell}\right) \hat{g}\left(s \lambda_{\ell}\right)
$$

Applying the inverse graph Fourier transform yields

$$
T^{s} f=\sum_{\ell=1}^{n} \hat{f}\left(\lambda_{\ell}\right) \hat{g}\left(s \lambda_{\ell}\right) u_{\ell}
$$

In order to have $T^{s} f(\tau)=W_{f}(s, \tau)$, as in the classical wavelet transform, the graph wavelet in node $\tau$ at scale $s$ is defined by

$$
\psi_{s, \tau}:=T^{s} \delta_{\tau}
$$

where $\delta_{\tau}$ is the delta signal (signal that assigns value one to node $\tau$ and zero to the other nodes). Thus,

$$
\psi_{s, \tau}=\sum_{\ell=1}^{n} \widehat{\delta}_{\tau}\left(\lambda_{\ell}\right) \hat{g}\left(s \lambda_{\ell}\right) u_{\ell}
$$

Since

$$
\widehat{\delta}_{\tau}\left(\lambda_{\ell}\right)=\sum_{i=1}^{n} \delta_{\tau}\left(\tau_{i}\right) u_{\ell}\left(\tau_{i}\right)=u_{\ell}(\tau)
$$

we have

$$
\psi_{s, \tau}=\sum_{\ell=1}^{n} u_{\ell}(\tau) \hat{g}\left(s \lambda_{\ell}\right) u_{\ell}
$$

Let $U=\left[u_{1}\left|u_{2}\right| \ldots \mid u_{n}\right]$ be a matrix whose columns are given by the graph Laplacian eigenvectors $u_{\ell}$ and $D_{\hat{g}}=\left(d_{i j}\right)$ be a diagonal matrix with entries $d_{i i}=\hat{g}\left(s \lambda_{i}\right)$. With these definitions, the graph wavelet $\psi_{s, \tau}$ can be written in matrix form

$$
\psi_{s, \tau}=U D_{\hat{g}} U^{T} \delta_{\tau}
$$


The graph wavelet coefficients are formally given by the inner product between the signal and the graph wavelets

$$
W_{f}(s, \tau)=\left\langle f, \psi_{s, \tau}\right\rangle
$$

Using the inverse graph Fourier transform of $f$ and the fact that $\left\{u_{\ell} \mid \ell=1, \ldots, n\right\}$ form an orthonormal set

$$
\begin{aligned}
W_{f}(s, \tau) & =\left\langle f, \psi_{s, \tau}\right\rangle \\
& =\left\langle\sum_{\ell=1}^{n} \hat{f}\left(\lambda_{\ell}\right) u_{\ell}, \sum_{\ell=1}^{n} u_{\ell}(\tau) \hat{g}\left(s \lambda_{\ell}\right) u_{\ell}\right\rangle \\
& =\sum_{\ell=1}^{n} \hat{g}\left(s \lambda_{\ell}\right) \hat{f}\left(\lambda_{\ell}\right) u_{\ell}(\tau) \\
& =T^{s} f(\tau) .
\end{aligned}
$$

In summary, the wavelet coefficient in node $\tau$ at scale $s$ can be calculated by

$$
W_{f}(s, \tau)=\sum_{\ell=1}^{n} \hat{g}\left(s \lambda_{\ell}\right) \hat{f}\left(\lambda_{\ell}\right) u_{\ell}(\tau)
$$

and $T^{s} f(\tau)=W_{f}(s, \tau)$ exactly as intended. The function $\hat{g}(s \lambda)$ in Equation 3.31 is called wavelet kernel. The graph wavelet transform is then determined by the kernel $\hat{g}: \mathbb{R}^{+} \rightarrow \mathbb{R}^{+}$. Hammond et al. (HAMMOND; VANDERGHEYNST; GRIBONVAL, 2011) proposed as kernel the function

$$
\hat{g}(x)=\left\{\begin{array}{cl}
x^{2} & \text { for } 0 \leq x<1 \\
-5+11 x-6 x^{2}+x^{3} & \text { for } 1 \leq x \leq 2 \\
4 x^{-2} & \text { for } 2<x
\end{array}\right.
$$

For small values of $s$, the wavelet kernel $\hat{g}(s \lambda)$ accentuates high frequency eigenvectors, ensuring good localization, while large values of $s$ compress the wavelet kernel $\hat{g}(s \lambda)$ around low frequency eigenvectors to encode coarser description of local neighborhoods. In more practical terms, the scales used to generate the wavelet kernels are logarithmically sampled between $s_{1}$ and $s_{r}\left(s_{1}, s_{2}, \ldots, s_{r}\right)$, where $r$ is the number of scales, $s_{1}=2 / \lambda_{n}$, and $s_{r}=40 / \lambda_{n}$.

Another kernel $\hat{g}$ used to define the wavelet kernels is available in the literature. Tremblay and Borgnat (TREMBLAY; BORGNAT, 2014) modify the parameters in the original definition of the kernel $\hat{g}$ (HAMMOND; VANDERGHEYNST; GRIBONVAL, 2011) to create graph wavelets adapted for community mining.

Scaling function: Hammond et al. (HAMMOND; VANDERGHEYNST; GRIBONVAL, 2011) introduce an additional function to stably represent low frequency eigenvectors, the scaling 
function. This waveform is constructed by a real-valued function $\hat{h}$ that acts as a low-pass kernel with

$$
\hat{h}(x)=\gamma \exp \left(-\left(\frac{10 x}{0.3 \lambda_{n}}\right)^{4}\right)
$$

The parameter $\gamma$ is chosen such that $\hat{h}(0)$ is equal to the maximum value of $\hat{g}$. The scaling function coefficient is then given by

$$
S_{f}(\tau)=\sum_{\ell=1}^{n} \hat{h}\left(\lambda_{\ell}\right) \hat{f}\left(\lambda_{\ell}\right) u_{\ell}(\tau)
$$

For each node $\tau \in V$, the spectral graph wavelet transform produces a scaling function coefficient $S_{f}(\tau)$ and a sequence of wavelet coefficients $W_{f}\left(s_{1}, \tau\right), \ldots, W_{f}\left(s_{r}, \tau\right)$. The interpretation of the scaling function coefficient is identical to the other wavelet coefficients, but representing the lowest wavelet frequency. For simplicity, we refer to this combination of the scaling function coefficient and wavelet coefficients as "wavelet coefficients", which we assumed ordered from lowest to highest wavelet frequency $\left(S_{f}(\tau), W_{f}\left(s_{r}, \tau\right), \ldots, W_{f}\left(s_{1}, \tau\right)\right)$. In our implementation, we considered the scaling function and seven wavelet kernels (Figure 11).

To illustrate the above concept, consider the signal $f_{0}$ defined previously in Section 3.2 (Figure 12). Figure 12 depicts the wavelet coefficients associated with nodes $\tau_{15}$ and $\tau_{45}$, where the taller the bar, the larger the coefficient. The wavelet coefficients corresponding to low frequencies ( 1 to 3 ) in node $\tau_{15}$ are larger than high frequency coefficients (6 to 8 ), reflecting the fact that the signal in node $\tau_{15}$ is smoother. In contrast, larger coefficients appear in high frequencies in node $\tau_{45}$, indicating that the signal oscillates more abruptly on and around this node. Therefore, the information provided by the graph wavelet transform is more local than that given by the graph Fourier transform.

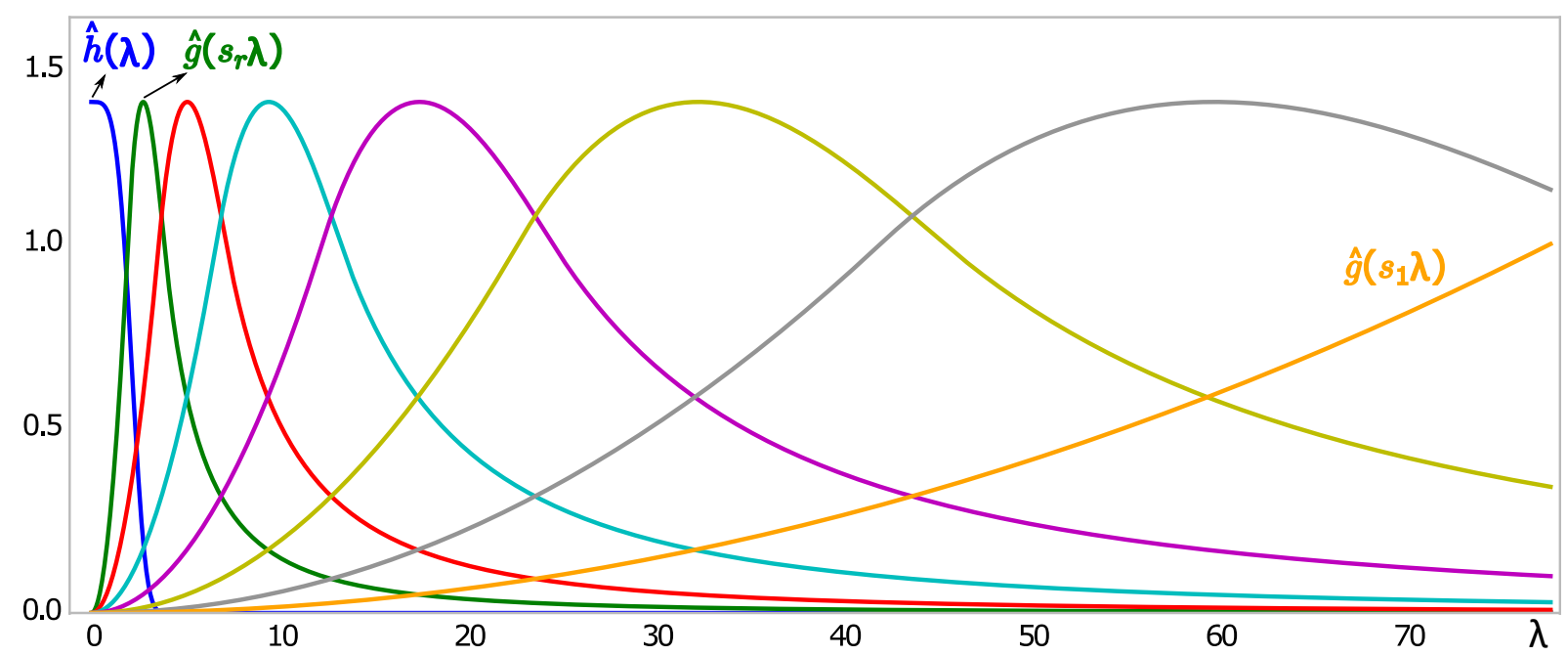

Figure 11 - Scaling function and seven wavelet kernels. 


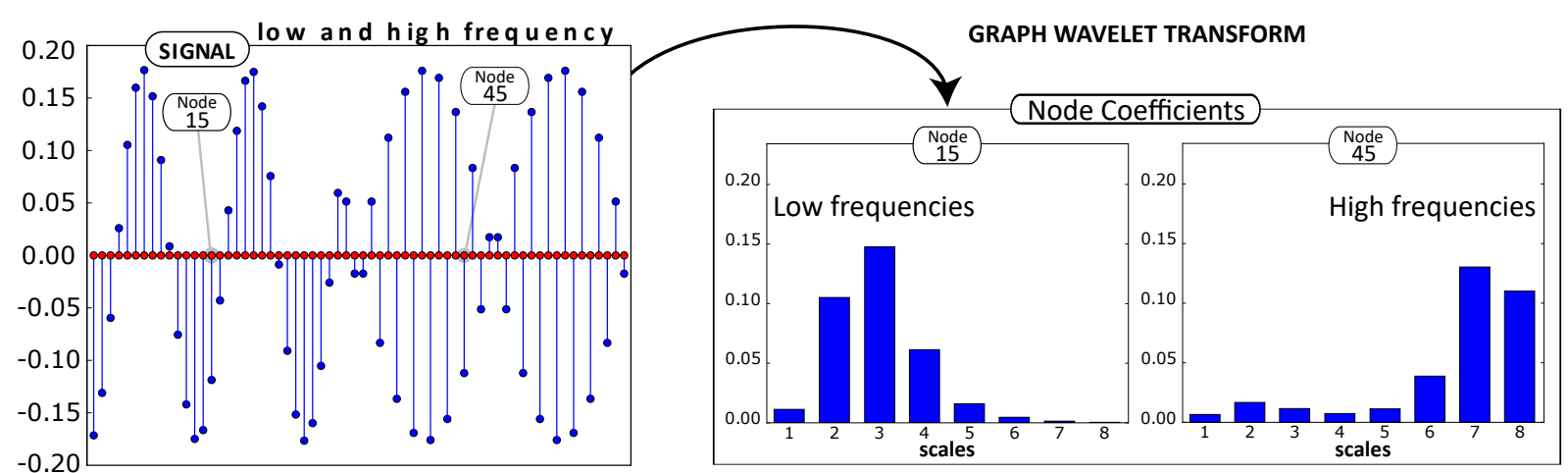

Figure 12 - Graph wavelet transform of signal $f_{0}$. Signal $f_{0}$ defined on the path graph with 64 nodes (left) and wavelet coefficients of nodes $\tau_{15}$ and $\tau_{45}$ (right).

\subsection{Fast Spectral Graph Wavelet Transform}

Calculating the wavelet coefficients from Equation 3.31 can become unfeasible when dealing with large graphs (graphs with hundreds of thousands or millions of nodes), since the whole set of eigenvalues and eigenvectors has to be computed, a task with computational cost of $O\left(n^{3}\right)$ that requires $O\left(n^{2}\right)$ memory, where $n$ is the number of nodes in $G$. However, the wavelet coefficients can be approximated using a scheme based on Chebyshev polynomials. The Chebyshev polynomials are given by the recursive formula

$$
T_{k}=2 y T_{k-1}-T_{k-2}
$$

with initial conditions $T_{0}=1$ and $T_{1}=y$, for $y \in[-1,1]$. These polynomials can be shifted to adjust their domains to the interval $\left[0, \lambda_{n}\right]$ by simply making

$$
\bar{T}_{k}(x)=T_{k}\left(\frac{x-a}{a}\right)
$$

with $a=\lambda_{n} / 2$ ( $\lambda_{n}$ is the largest graph Laplacian eigenvalue). The polynomials $\bar{T}_{k}$ are called shifted Chebyshev polynomials and they satisfy

$$
\bar{T}_{k}=2\left(\frac{y-a}{a}\right) \bar{T}_{k-1}-\bar{T}_{k-2}
$$

Since the shifted Chebyshev polynomials form an orthogonal basis for the Hilbert space of square integrable functions defined on the interval $\left[0, \lambda_{n}\right]$ (HAMMOND; VANDERGHEYNST; GRIBONVAL, 2011), for any scale $s$

$$
\hat{g}(s \lambda)=\frac{1}{2} c_{0}+\sum_{k=1}^{\infty} c_{k} \bar{T}_{k}(\lambda)
$$


where $c_{k}=\frac{2}{\pi} \int_{0}^{\pi} \cos (k \theta) \hat{g}(\operatorname{si}(\cos (\theta)+1)) d \theta$. Applying the operator $\bar{T}_{k}(L)$, where $L$ is the graph Laplacian, on the signal $f$ yields a vector $\bar{T}_{k}(L) f$ that satisfies the recursive formula

$$
\bar{T}_{k}(L) f=\frac{2}{a}(L-a I) \bar{T}_{k-1}(L) f-\bar{T}_{k-2}(L) f
$$

for $2 \leq k \leq m$, with initial conditions

$$
\bar{T}_{0}(L) f=f \text { and } \bar{T}_{1}(L) f=\frac{1}{a}(L-a I) f,
$$

where $I$ is the identity matrix. The demonstrations of Equations 3.39 and 3.40 are in Appendix A. Additionally, we also prove in Appendix A the following equality

$$
\bar{T}_{k}(L) f=\sum_{\ell=1}^{n} \bar{T}_{k}\left(\lambda_{\ell}\right) \hat{f}\left(\lambda_{\ell}\right) u_{\ell} .
$$

Thus, the wavelet coefficient in a node $\tau$ at scale $s$ can be approximated by

$$
\begin{aligned}
W_{f}(s, \tau) & =\sum_{\ell=1}^{n} \hat{g}\left(s \lambda_{\ell}\right) \hat{f}\left(\lambda_{\ell}\right) u_{\ell}(\tau) \\
\stackrel{(3.38)}{=} & \sum_{\ell=1}^{n}\left[\frac{1}{2} c_{0}+\sum_{k=1}^{\infty} c_{k} \bar{T}_{k}\left(\lambda_{\ell}\right)\right] \hat{f}\left(\lambda_{\ell}\right) u_{\ell}(\tau) \\
& \approx \sum_{\ell=1}^{n}\left[\frac{1}{2} c_{0}+\sum_{k=1}^{m} c_{k} \bar{T}_{k}\left(\lambda_{\ell}\right)\right] \hat{f}\left(\lambda_{\ell}\right) u_{\ell}(\tau) \\
& =\frac{1}{2} c_{0} \sum_{\ell=1}^{n} \hat{f}\left(\lambda_{\ell}\right) u_{\ell}(\tau)+\sum_{k=1}^{m} c_{k} \sum_{\ell=1}^{n} \bar{T}_{k}\left(\lambda_{\ell}\right) \hat{f}\left(\lambda_{\ell}\right) u_{\ell}(\tau) \\
\stackrel{(3.41)}{=} & \left(\frac{1}{2} c_{0} f+\sum_{k=1}^{m} c_{k} \bar{T}_{k}(L) f\right)(\tau) .
\end{aligned}
$$

In summary, the approximation is given by

$$
W_{f}(s, \tau) \approx\left(\frac{1}{2} c_{0} f+\sum_{k=1}^{m} c_{k} \bar{T}_{k}(L) f\right)(\tau),
$$

where $m$ is a truncation parameter, $\hat{g}$ is the kernel as defined in Equation 3.32. With a completely analogous calculation, the scaling function coefficient can be approximated by

$$
S_{f}(\tau) \approx\left(\frac{1}{2} d_{0} f+\sum_{k=1}^{m} d_{k} \bar{T}_{k}(L) f\right)(\tau)
$$

with $d_{k}=\frac{2}{\pi} \int_{0}^{\pi} \cos (k \theta) \hat{h}(a(\cos (\theta)+1)) d \theta$ (Proof in Appendix A). 
Computational Cost \& Memory Usage: Equation 3.47 shows that the wavelet coefficient in node $\tau$ at scale $s$ can be obtained by computing only the largest graph Laplacian eigenvalue $\lambda_{n}$ rather then the whole set of eigenvalues and eigenvectors. The computation of $\left(L \bar{T}_{k-1}(L) f\right)(i)$ (the $i$-th entry of the vector $\left.L \bar{T}_{k-1}(L) f\right)$ on the right side of Equation 3.39 involves only the neighbors of node $\tau_{i}$ on $G$, avoiding the explicit construction of the graph Laplacian. Therefore, the computational cost to approximate the wavelet coefficients at a given scale is $O(m|E|+m n)$, with $m|E|$ corresponding to the calculation of the vectors $\bar{T}_{k}(L) f$ via recursive formula in Equation 3.39 and $m n$ to the calculation of the coefficients in Equation 3.47, where $|E|$ is the number of edges in $G$. Furthermore, the memory to approximate the wavelet coefficients at a particular scale is $O(3 n+n)$, with $3 n$ corresponding to the application of recursive formula in Equation 3.39 and $n$ to the calculation of the coefficients in Equation 3.47.

The truncation parameter $m$ dictates the quality of the approximation, and it should be tuned for a good trade-off between accuracy and computational cost. Figure 13 depicts the processing time and error in the approximation of the wavelet coefficients for a synthetic network (see Section 5.4), using an i7 processor. As one can see, $m=40$ is a good trade off. The same trade-off result was observed in other tests we carried out, leading us to assume this parameter value in all of our experiments. Shuman et al. (SHUMAN; VANDERGHEYNST; FROSSARD, 2011) suggest that $m$ equal to 20 results in a close approximation. However, with a slight increase in processing time, it is possible to increase $m$ significantly reducing the error.

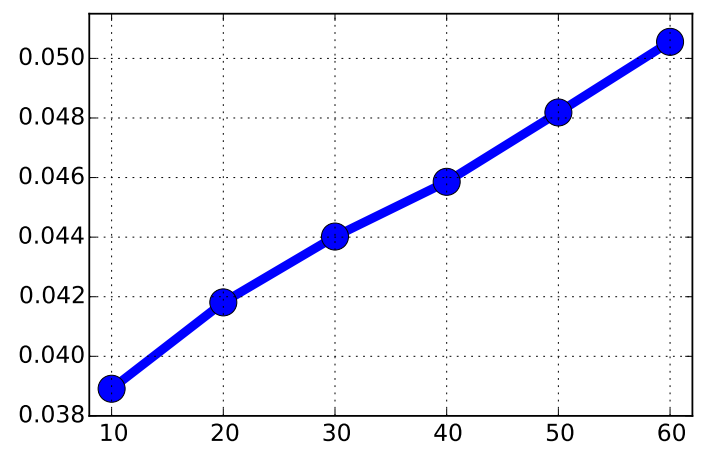

(a) Time (seconds)

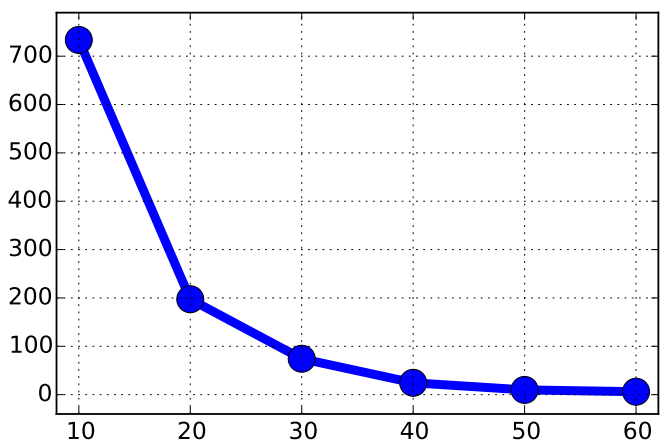

(b) Error

Figure 13 - Computational cost and error in the approximation of the wavelet coefficients for a synthetic network as a function of $m$. (a) Processing times. (b) Sum of absolute differences between exact and approximated wavelet coefficients.

The graph wavelet transform takes into account a neighborhood that reaches beyond the direct neighbors. For instance, the signal in the light green nodes in Figure 14 is in unison with second-order neighbors and the wavelet coefficients gracefully react to this phenomenon. 

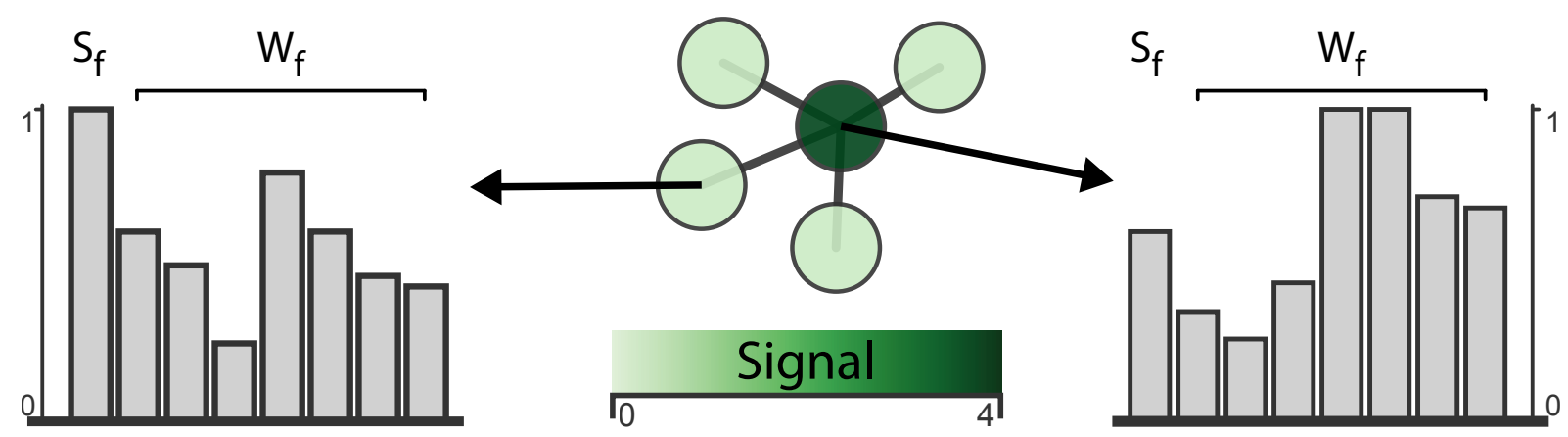

Figure 14 - An example of graph with low and high frequency nodes. The wavelet coefficients allow to infer the signal behavior on and around each node. 

CHAPTER

\section{4}

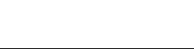

\section{ILLUSTRATIVE INTERPRETATION OF GRAPH WAVELETS}

Defined as a generalization of the classical wavelet transform in Chapter 3, the graph wavelet transform can be reinterpreted using the concept of dictionary of graph spectral filters. A graph spectral filter, or kernel, $\hat{g}: \Lambda \rightarrow \mathbb{R}$ is a function defined on the spectral domain $(\Lambda=$ $\left\{\lambda_{1}, \lambda_{2}, \ldots, \lambda_{n}\right\}$ ), which associates a scalar $\hat{g}\left(\lambda_{\ell}\right)$ with each eigenvalue $\lambda_{\ell} \in \Lambda$. The graph Fourier transform $\hat{f}: \Lambda \rightarrow \mathbb{R}$ can be seen as a particular instance of graph spectral filter. A dictionary of graph spectral filters $\left\{\hat{g}_{m} \mid m=1,2, \ldots, M\right\}$ is a set of graph spectral filters $\hat{g}_{m}: \Lambda \rightarrow \mathbb{R}$, $m=1,2, \ldots, M$, where $M$ is the number of graph spectral filters in the dictionary.

Given a signal $f$ and a dictionary $\left\{\hat{g}_{m} \mid m=1,2, \ldots, M\right\}$, a spectral graph wavelet transform $W_{f}:\{1,2, \ldots, M\} \times V \rightarrow \mathbb{R}$ can be defined by

$$
W_{f}(m, \tau)=\sum_{\ell=1}^{n} \hat{g}_{m}\left(\lambda_{\ell}\right) \hat{f}\left(\lambda_{\ell}\right) u_{\ell}(\tau)
$$

By varying $m$ while keeping $\tau$ fixed, we obtain $M$ wavelet coefficients associated with node $\tau$

$$
W_{f}(1, \tau), W_{f}(2, \tau), \ldots, W_{f}(M, \tau)
$$

Selecting the graph spectral filter $\hat{g}_{m}$ as a band-pass filter, where small values of $m$ correspond to low-pass filters and large values to high-pass filters, makes the coefficients encode the behavior of the signal on each node. For instance, choosing the dictionary of graph spectral filters equal to

$$
\left\{\hat{h}(\lambda), \hat{g}\left(s_{r} \lambda\right), \ldots, \hat{g}\left(s_{1} \lambda\right)\right\}
$$

where the kernel $\hat{g}$, scaling function $\hat{h}$, and scales $s_{1}, \ldots, s_{r}$ are defined as in Chapter 3 , produces exactly the spectral graph wavelets (SGW) (HAMMOND; VANDERGHEYNST; GRIBONVAL, 
2011). In this chapter, the kernels and graph wavelets generated by this graph wavelet transform are denoted by $\hat{g}_{m}^{\text {Ham }}$ and $\psi_{m, \tau}^{\mathrm{Ham}}$, respectively. The corresponding dictionary (Equation 4.3) is called spectral graph wavelet dictionary, or succinctly SGW dictionary.

This chapter presents a generalization of the spectral graph wavelets, the spectrumadapted graph wavelets (SAGW) (SHUMAN et al., 2015). We explore main differences between both spectral graph wavelet transforms. Moreover, some remarkable properties of the graph wavelets are highlighted through illustrative examples, which use synthetic and real-world data. The source codes used to produce the results presented in this chapter are available on a GitHub page located at $<$ https://github.com/AlcebiadesDalColJunior/>.

\subsection{Spectrum-Adapted Graph Wavelet Transform}

Shuman et al. (SHUMAN et al., 2015) initially propose the construction of a dictionary of uniform translates. The uniform translates are translated versions of each other uniformly distributed on the spectral domain. In more mathematical terms, there is a function $\hat{g}^{U}$ and a constant $a$ such that the graph spectral filters $\hat{g}_{m}$ can be written by

$$
\hat{g}_{m}(\lambda)=\hat{g}^{U}(\lambda-m a), \forall \lambda \in\left[0, \lambda_{n}\right]
$$

for $m=1,2, \ldots, M$, where $\lambda_{n}$ is the largest graph Laplacian eigenvalue. It is important to note, the graph spectral filters $\hat{g}_{m}, m=1,2, \ldots, M$ are defined in the interval $\left[0, \lambda_{n}\right]$, possibly cutting part of the original function $\hat{g}^{U}$. Figure 15 depicts a dictionary of uniform translates with eight graph spectral filters.

The uniform translates are then modified to adapt itself to the spectrum of a given graph. The density of the graph Laplacian eigenvalues is estimated using a fast approximation mechanism, and the larger the number of eigenvalues in a certain region of the spectral domain, the narrower the graph spectral filters are in that region. In Figure 16, we simulate four graph Laplacian spectra to illustrate the way the uniform translates follow the distribution of the graph Laplacian eigenvalues, which are indicated by $x$ markers on the horizontal axes.

The dictionary of uniform translates adaptable to the spectrum of a specific graph forms the spectrum-adapted graph wavelets (SAGW) (SHUMAN et al., 2015). This dictionary is called spectrum-adapted graph wavelet dictionary, or briefly SAGW dictionary. In addition, the kernels and graph wavelets generated by this graph wavelet transform are denoted by $\hat{g}_{m}^{S h u}$ and $\psi_{m, \tau}^{S h u}$. More technical details about the construction of the uniform translates (Equation 4.4) and the method used to modify them can be found in (SHUMAN et al., 2015).

Before going into more practical details in the next section, we address some theoretical differences of the spectral graph wavelet transforms. In this regard, consider the following definitions of frame and tight frame. 


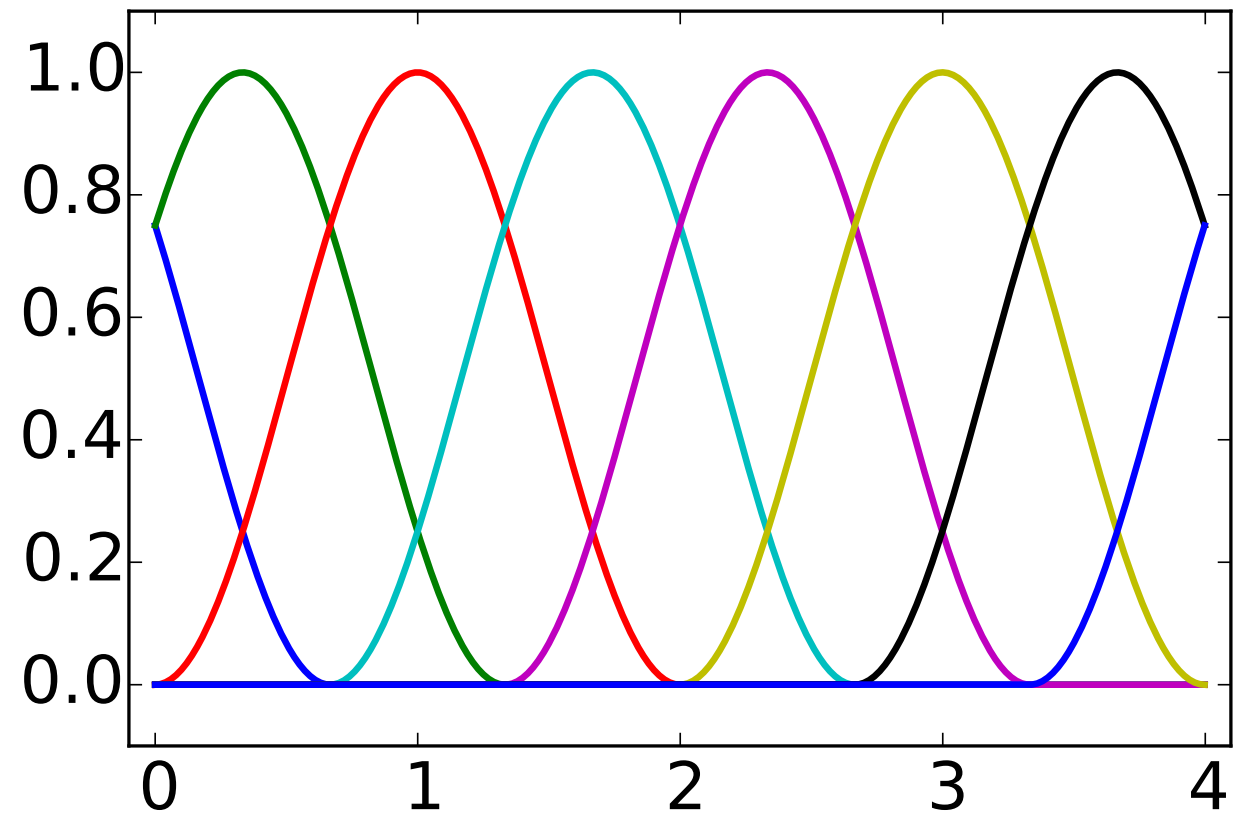

Figure 15 - Dictionary of uniform translates.

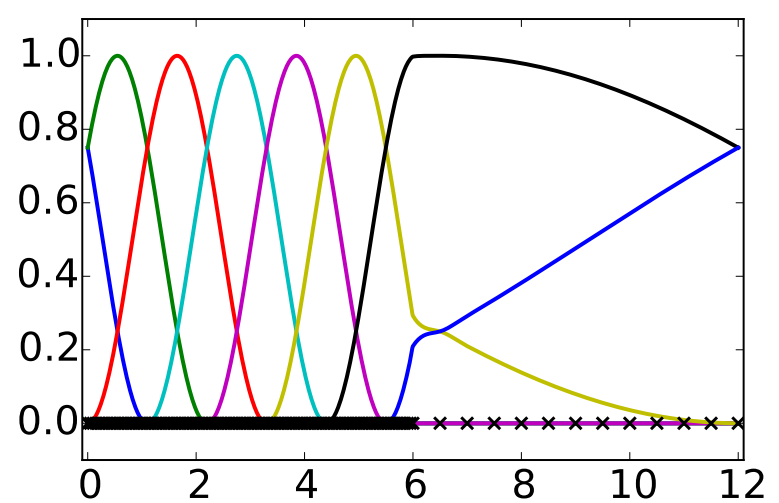

(a)

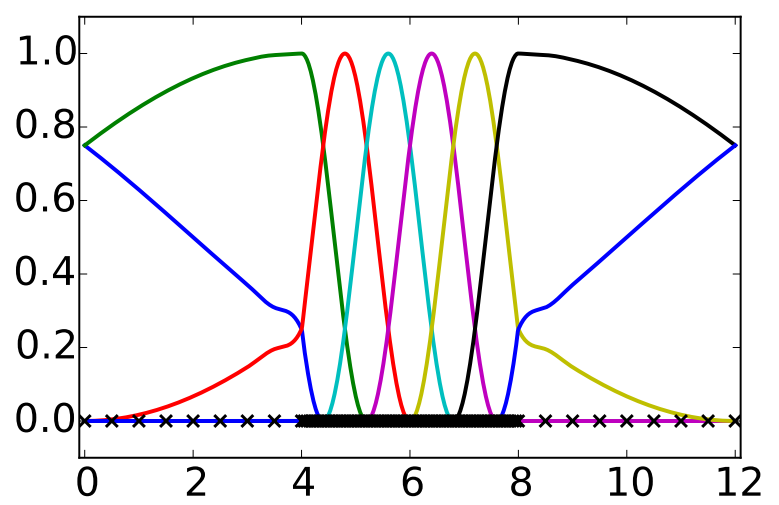

(c)

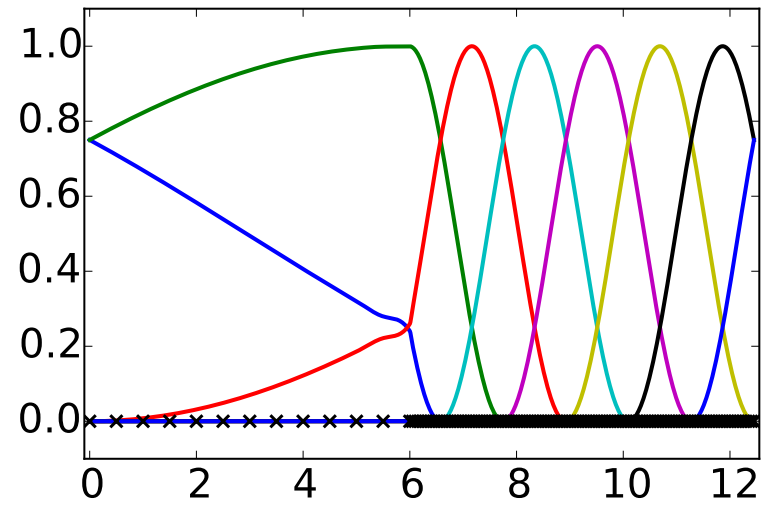

(b)

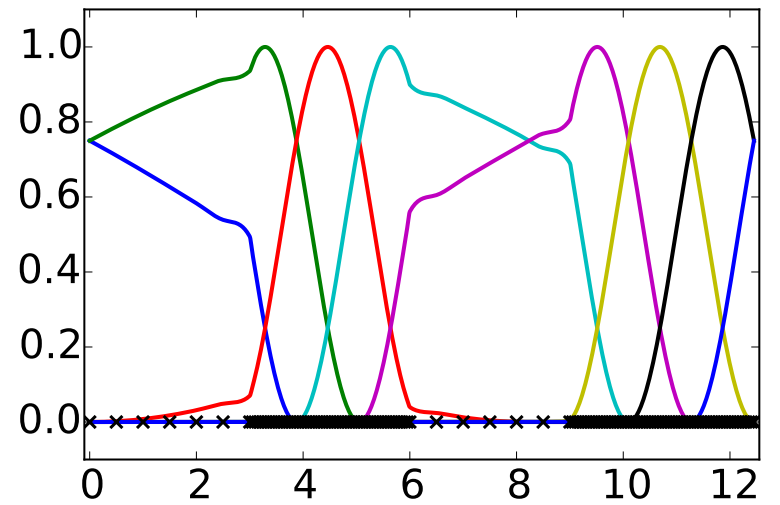

(d)

Figure 16 - Uniform translates adapting to graph Laplacian spectra. 
Definition 1 (Frame): A set $\left\{\Gamma_{k}\right\}$ forms a frame if there are numbers $A, B>0$ satisfying

$$
A\|f\|^{2} \leq \sum_{k}\left|\left\langle f, \Gamma_{k}\right\rangle\right|^{2} \leq B\|f\|^{2},
$$

for all $f$. In this case, the numbers $A$ and $B$ are called frame bounds.

Definition 2 (Tight frame): A frame $\left\{\Gamma_{k}\right\}$ is a tight frame if the frame bounds $A$ and $B$ are equal $(A=B)$.

The spectral graph wavelets form a frame (HAMMOND; VANDERGHEYNST; GRIBONVAL, 2011) with frame bounds

$$
\begin{aligned}
& A=\min _{\lambda \in\left[0, \lambda_{n}\right]} G(\lambda), \\
& B=\max _{\lambda \in\left[0, \lambda_{n}\right]} G(\lambda),
\end{aligned}
$$

where

$$
\begin{aligned}
G(\lambda) & =\sum_{m=1}^{M} \hat{g}_{m}^{\text {Ham }}(\lambda)^{2} \\
& =\hat{h}(\lambda)^{2}+\sum_{j=1}^{r} \hat{g}\left(s_{j} \lambda\right)^{2} .
\end{aligned}
$$

In words, $G$ is basically the sum of squares of the graph spectral filters. A frame ensures that every signal $f$ can be recovered iteratively from the wavelet coefficients (HEIL; WALNUT, 1989). This remarkable property of the spectral graph wavelets allows more than just signal analysis, one can manipulate the wavelet coefficients of a signal and then invert the graph wavelet transform.

On the other hand, the spectrum-adapted graph wavelets form a tight frame (SHUMAN et al., 2015) with frame bounds

$$
\begin{aligned}
& A=n \cdot \min _{\lambda \in\left[0, \lambda_{n}\right]} G(\lambda), \\
& B=n \cdot \max _{\lambda \in\left[0, \lambda_{n}\right]} G(\lambda),
\end{aligned}
$$

where

$$
G(\lambda)=\sum_{m=1}^{M} \hat{g}_{m}^{S h u}(\lambda)^{2}
$$


In order to make the spectrum-adapted graph wavelets a tight frame, the spectral graph filters are built so as to the function $G$ is always a constant function $\left(G(\lambda)=c, \forall \lambda \in\left[0, \lambda_{n}\right]\right)$. Figure 17 illustrates the sum of squares of the SGW and SAGW dictionaries for the path graph with 64 nodes, the black top curves. Even though, the kernels in the SAGW dictionary adapt to the spectrum of the path graph, the function $G$ remains constant.

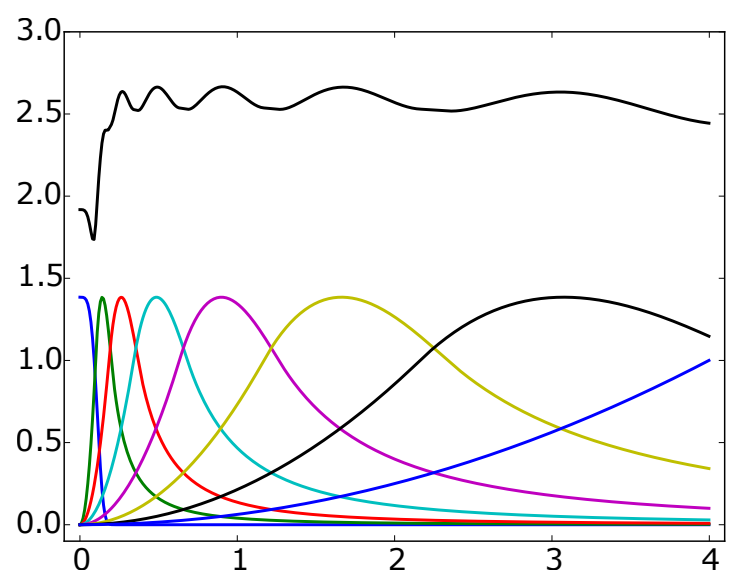

(a) SGW

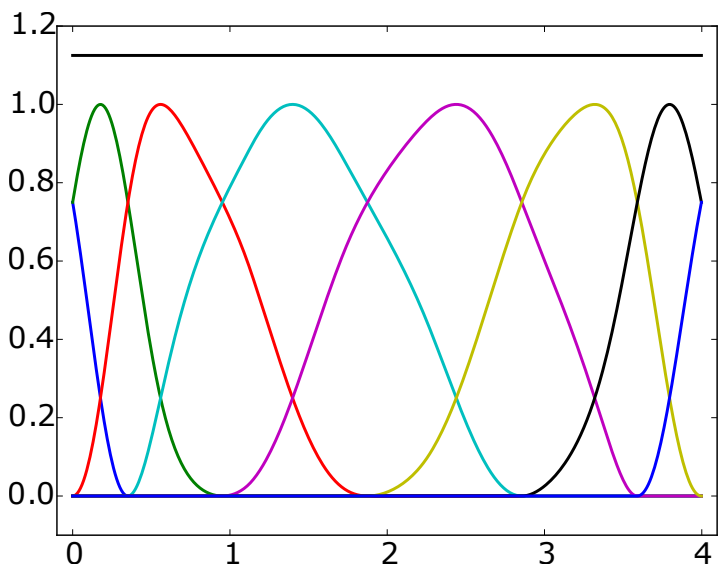

(b) SAGW

Figure 17 - Sum of squares of the SGW and SAGW dictionaries for a path graph.

Forming a tight frame ensures additional properties to the graph wavelet transform. Indeed, let $\left\{\Gamma_{k}\right\}$ represents the spectrum-adapted graph wavelets $\left\{\psi_{m, \tau}^{S h u}\right\}$, then

$$
A\|f\|^{2} \leq \sum_{k}\left|\left\langle f, \Gamma_{k}\right\rangle\right|^{2} \leq B\|f\|^{2},
$$

with the frame bounds satisfying $A=B$. Thereby, the two inequalities in Equation (4.11) and the equality $A=B$ guarantee

$$
\sum_{k}\left\langle f, \Gamma_{k}\right\rangle^{2}=A\|f\|^{2}
$$

Since the wavelet coefficients are given by the inner product of the signal with the graph wavelets, we can write

$$
\begin{aligned}
\sum_{m} \sum_{\tau} W_{f}(m, \tau)^{2} & =\sum_{m} \sum_{\tau}\left\langle f, \psi_{m, \tau}^{S h u}\right\rangle^{2} \\
& =\sum_{k}\left\langle f, \Gamma_{k}\right\rangle^{2} \\
& =A\|f\|^{2} .
\end{aligned}
$$

In other words, the squared magnitudes of the wavelet coefficients can be interpreted as an energy density function, making the understanding of the wavelet coefficients a more intuitive task. 


\subsection{Practical Differences between the Spectral Graph Wavelet Transforms}

In this section, we employ a comet graph to highlight important differences between the spectral graph wavelets and spectrum-adapted graph wavelets. A comet graph is a graph formed combining a path graph and a star graph, the latter centered in one of the extremities of the path graph. Figure 18 depicts a comet graph. The graph Laplacian eigenvalues of a comet graph have a peculiar distribution, as the difference between the two largest eigenvalues is much larger than the difference between the other eigenvalues (Figure 18). Although the second largest eigenvalue is distant from the largest eigenvalue, it corresponds to the second highest frequency in the spectrum of the comet graph.

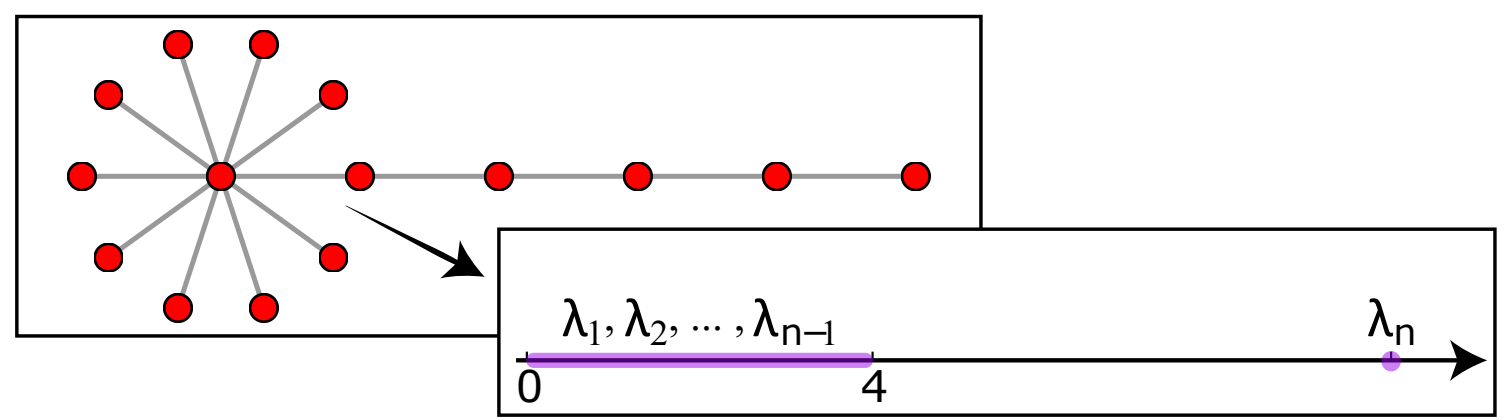

Figure 18 - Comet graph and corresponding spectral distribution. The purple interval concentrates $n-1$ graph Laplacian eigenvalues. The largest eigenvalue is isolate; the greater the degree of the star graph, the greater the distance.

As discussed previous, each graph spectral filter $\hat{g}_{m}$ operates as a band-pass filter on the spectral domain, thus the distribution of the eigenvalues impacts in which frequencies are filtered. Figure 19 depicts the spectral graph wavelet and spectrum-adapted graph wavelet dictionaries, both defined in the same spectral domain, the spectrum of the comet graph. The graph wavelet transform derived from each dictionary are quite different, since the SGW dictionary is designed to evenly cover the spectral domain and the SAGW dictionary adapts to the spectral distribution. As one can clearly see in Figure 19, when eigenvalues are not evenly distributed on the spectral domain, as is the case of the comet graph, the SAGW dictionary better fits the frequency bands.

To illustrate practical differences between the transforms, we create a comet graph with 64 nodes whose star extremity contains 30 nodes. The signal is defined as the second largest graph Laplacian eigenvector (signal panel in Figure 20). Figure 20 illustrates the graph Fourier transform of this signal. As expected, the graph Fourier transform presents a spike in the second to last eigenvalue. Figure 20 also depicts the wavelet coefficients of the specified node computed using both graph wavelet transforms. The spectral graph wavelet dictionary led to wavelet coefficients with dominant frequencies in the central frequencies (4 and 5), not properly characterizing the high frequency nature of the signal under analysis. In contrast, the 
spectrum-adapted graph wavelet dictionary generated wavelet coefficients with larger intensity in the coefficients related to high frequencies, better capturing the pattern of variation of the signal.

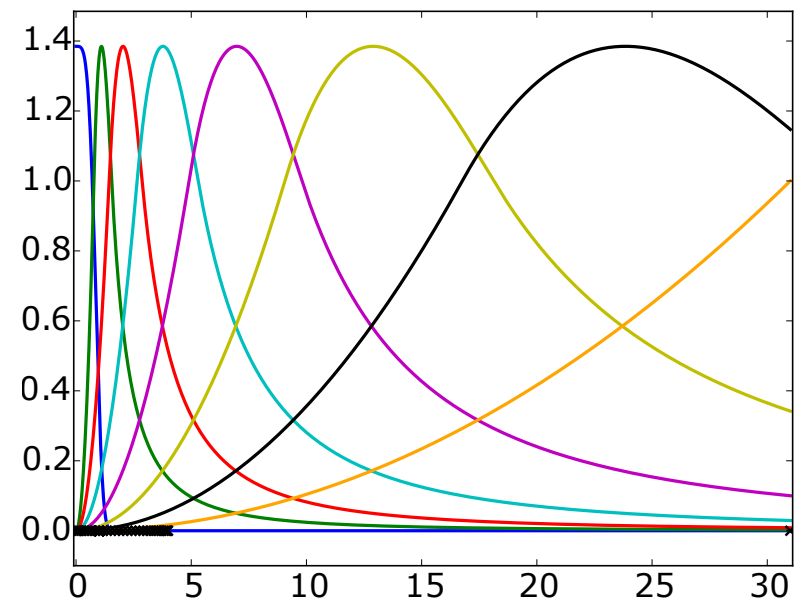

(a) $\mathrm{SGW}$

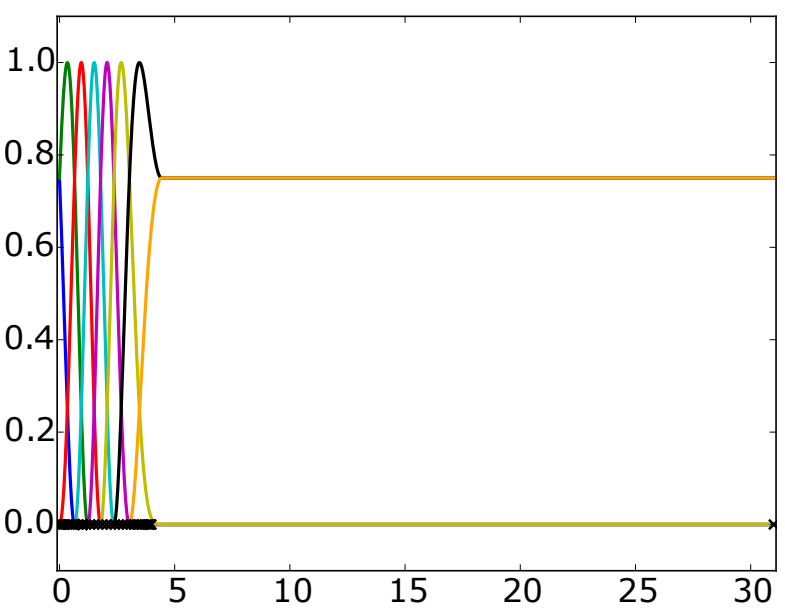

(b) SAGW

Figure 19 - Graph spectral filters of the SGW and SAGW dictionaries for a comet graph with 64 nodes. The $x$ marks on the horizontal axes represent the graph Laplacian spectrum of the comet graph. The kernels of the SAGW dictionary adapt to regions with larger spectral density.

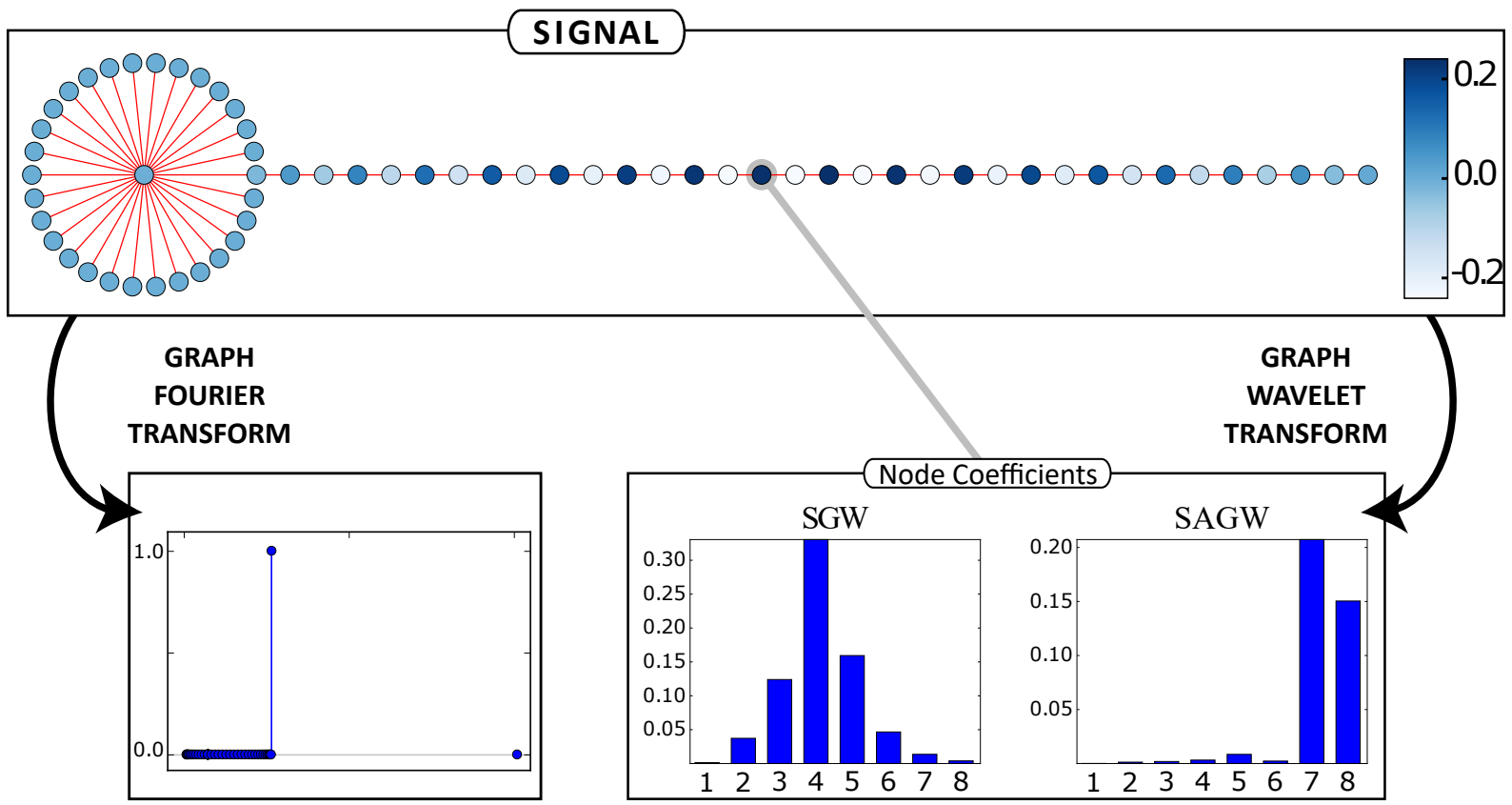

Figure 20 - Second largest graph Laplacian eigenvector defined on a comet graph with 64 nodes (top). The color associated with the graph nodes encodes the graph signal. The arrows indicate the corresponding graph Fourier transform and wavelet coefficients of the specified node. The graph Fourier transform reveals the presence of high frequency. The wavelet coefficients were produced using the SGW and SAGW dictionaries. 


\subsection{Interplay among Topology, Spectrum, and Wavelet Coefficients}

The example involving the comet graph in the previous section, helped to understand how the choice of the graph wavelet transform affects the wavelet coefficients. Let's analyze now how patterns in the signal are captured by the wavelet coefficients in a more complicated topology, the topology of the Minnesota road graph.

Consider the Minnesota road graph (GLEICH, 2015) as depicted in Figure 21. We divide the graph into three different regions $R_{1}, R_{2}$, and $R_{3}$, colored as blue, red, and green in the cluster panel of Figure 21.

A signal with a different dominant frequency band is assigned to each region, resulting in a global signal defined by

$$
f=\sum_{j=1}^{3} \frac{f_{j}}{\left\|f_{j}\right\|_{\infty}}
$$

where

$$
f_{j}(\tau)=\left\{\begin{array}{cl}
\sum_{\ell \in I_{j}} u_{\ell}(\tau) & \text { if } \tau \in R_{j} \\
0 & \text { otherwise }
\end{array}\right.
$$

and $I_{j}$ is the set of indexes of the eigenvalues lying in the intervals $\left[a_{j}, b_{j}\right]$. The sets $I_{1}, I_{2}$, and $I_{3}$ are given by $[0.0,0.08],[2.0,2.5]$, and [5.0, 7.0], respectively. The length of the intervals was chosen according to the distribution of the eigenvalues, as illustrated in the eigenvalue panel of Figure 21. Thereby, region $R_{1}$ (blue) has a low frequency signal, since the signal is

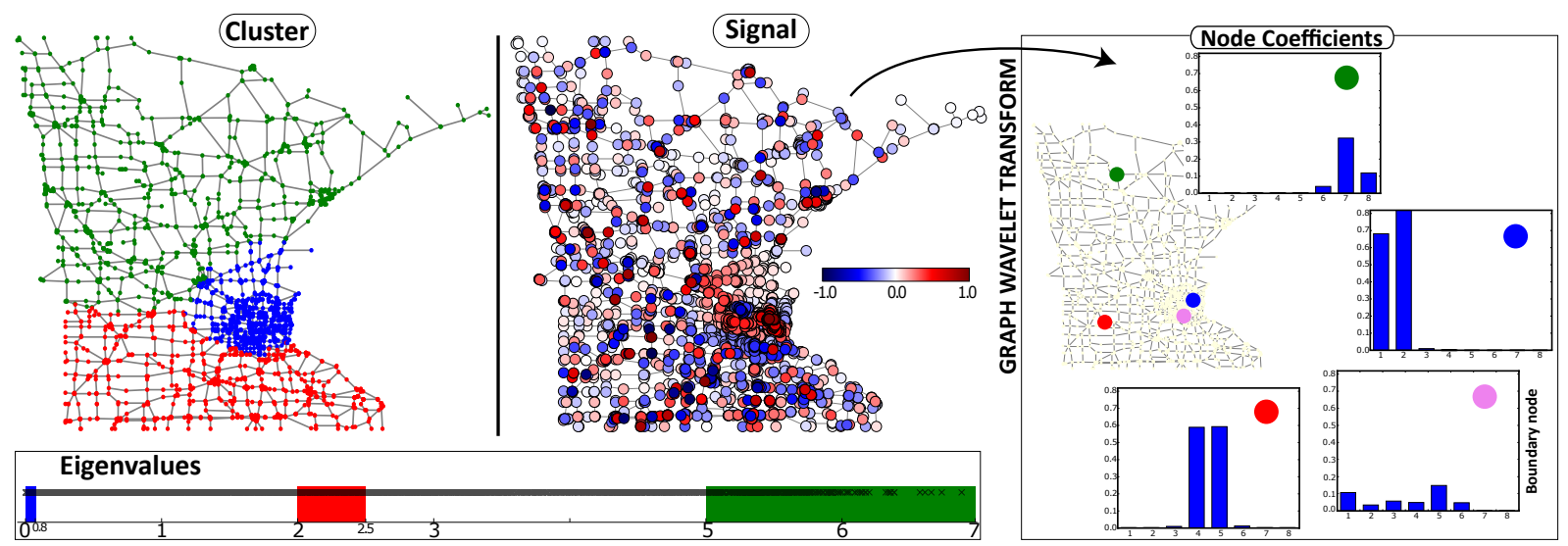

Figure 21 - Minnesota road graph divided into three different regions (cluster) and signal defined on the Minnesota road graph (signal). The signal is formed so that each region has a dominant frequency interval, intervals that are highlighted on eigenvalue panel. Wavelet coefficients of representative nodes (dot colors) and one node close to the boundary between regions (pink). The graph wavelet transform was produced using the SAGW dictionary. 
the summation of eigenvectors corresponding to eigenvalues in the low frequency region of the spectrum. Similarly, the signal on regions $R_{2}$ (red) and $R_{3}$ (green) corresponds to the summation of eigenvectors associated with median and high frequency eigenvalues, respectively. The signal panel of Figure 21 depicts the signal $f$ as defined above.

The node coefficient panel of Figure 21 depicts the wavelet coefficients of representative nodes for regions $R_{1}, R_{2}$, and $R_{3}$, computed using the SAGW dictionary. The behavior of the wavelet coefficients corresponds to exactly what we expect. However, the wavelet coefficients corresponding to representative nodes are observed mainly in the middle of regions $R_{1}, R_{2}$, and $R_{3}$. Nodes close to the boundary between regions tend to have a more mixed behavior, as illustrated in the node coefficient panel of Figure 21 with the pink node.

\subsection{Time-Varying Data Analysis via Graph Wavelets}

The discussion in Chapter 3 assumes a static signal defined on the nodes of the graph. However, in many applications data varies with time, resulting in a time series associated with each node of the graph (SANDRYHAILA; MOURA, 2014). One of the possible options to create a graph representing time-varying data is to use the Cartesian product of graphs.

Given two graphs $G=\left(V_{G}, E_{G}\right)$ and $H=\left(V_{H}, E_{H}\right)$, the Cartesian product of them is a new graph $G \times H$ with node set

$$
V_{G} \times V_{H}=\left\{\tau \imath \mid \tau \in V_{G}, \imath \in V_{H}\right\}
$$

and edges connecting nodes $\tau_{i} \boldsymbol{l}_{j}$ and $\tau_{k} \boldsymbol{l}_{l}$ iff

$$
\begin{aligned}
& \tau_{i}=\tau_{k} \text { and } \iota_{j} \text { is adjacent to } \iota_{l} \text { in } H, \text { or } \\
& \iota_{j}=\iota_{l} \text { and } \tau_{i} \text { is adjacent to } \tau_{k} \text { in } G .
\end{aligned}
$$

If $H$ is a path graph, the Cartesian product graph $G \times H$ corresponds to "stacked" copies of $G$ with edges connecting corresponding nodes in adjacent copies (Figure 22). Each copy of $G$ corresponds to a time slice of the temporal data, enabling the use of the graph wavelet transform on $G \times H$ to analyze time-varying data.

In the Cartesian product graph, the wavelet coefficients capture spatio-temporal behavior of the signal, making their interpretation more intricate than in the static case. In order to gain some intuition about the spatio-temporal signal variation captured by the wavelet coefficients, we present the following example.

Synthetic time-varying data: We initially consider a spatial graph $G$ as depicted in Figure 23 . On each node is defined a synthetic time series with nineteen time slices, so we design a Cartesian 


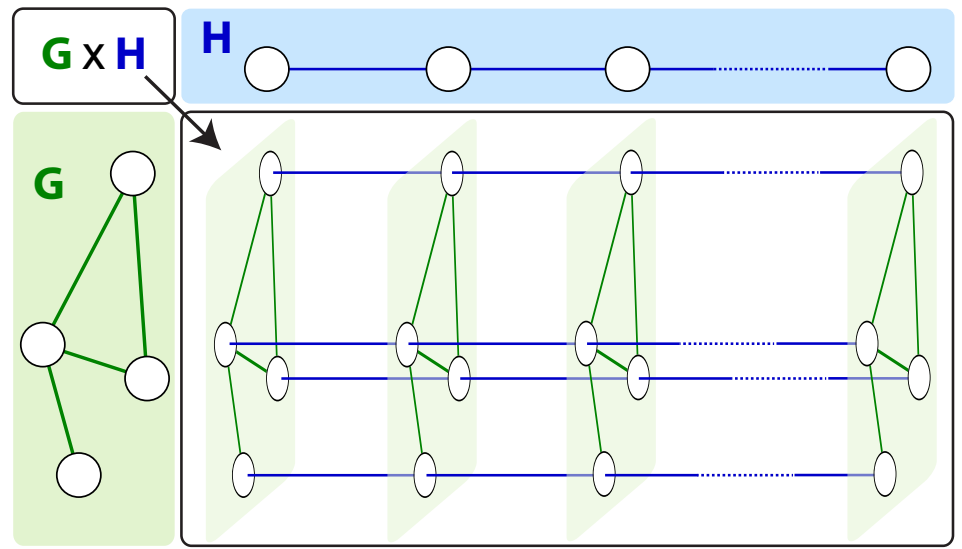

Figure 22 - Time-varying data modeled by the Cartesian product of two graphs: spatial $G$ and temporal $H$.

product graph $G \times H$ where $H$ is a path graph with nineteen nodes (Figure 23). In other words, graph $G \times H$ comprises nineteen time slices of time-varying data defined on its nodes. Given the Cartesian graph $G \times H$, all times series defined on the nodes of $G$ can be interpreted as a single signal defined on $G \times H$. We craft four different signals on $G \times H$ denoted as $f_{i}, i=1,2,3,4$. In the definition of the signals, we will need to know the nodes $\tau_{a} \in G$ and $\imath_{10} \in H$, which are enhanced in Figure 23.

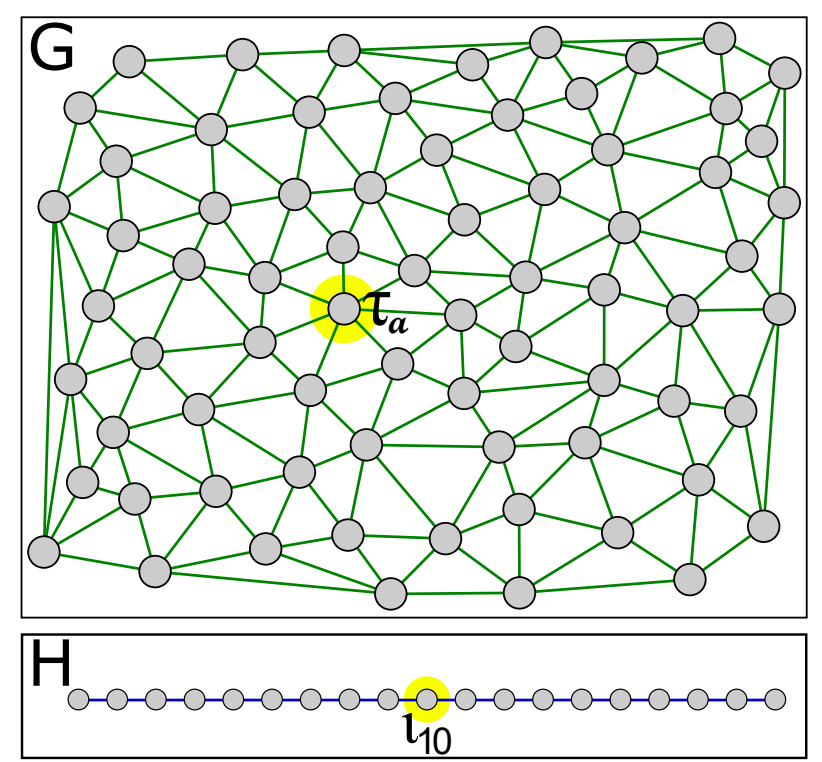

Figure 23 - Graphs: spatial $G$ and temporal $H$. Graph $H$ is the path graph with nineteen nodes.

The first signal $f_{1}$ is defined with a spike of value equal to 1 in node $\tau_{a} l_{10}$ of $G \times H$, vanishing on the remaining nodes. In other words, the time series in node $\tau_{a}$ has a spike in time slice 10 , and time series on the other nodes are all null. The second signal $f_{2}$ is defined as a "spatial Gaussian" centered in node $\tau_{a} l_{10}$, assigning values for nodes in the neighborhood of $\tau_{a}$ in time slice 10, and zero in other time slices. This signal corresponds to a smooth spatial event that lasts only an instant of time. The third signal $f_{3}$ is defined as a "temporal Gaussian" centered at node $\tau_{a} l_{10}$, assigning values for nodes $\tau_{a} l_{j}, j=1, \ldots, 19$, and zero elsewhere, thus representing 
a smooth temporal event that happens on a single node. Finally, signal $f_{4}$ is the combination of a Gaussian over time centered at $\tau_{a} l_{10}$ and Gaussians in all time slices with spikes on $\tau_{a} \boldsymbol{l}_{j}$, $j=1, \ldots, 19$. Signal $f_{4}$ simulates a smooth spatial event on $G$ that grows over time until time slice 10 and then decreases until completing the nineteen time slices.

Figure 24 depicts the wavelet coefficients associated with node $\tau_{a} \iota_{10}$. The coefficients correctly reflect the behavior of the signals on the selected node. In the bar chart corresponding to $f_{1}$, there is a concentration of large coefficients in high frequencies, indicating that $f_{1}$ has a high frequency on $\tau_{a} l_{10}$. The smoothness of $f_{2}$ and $f_{4}$ in node $\tau_{a} l_{10}$ is also evident in the bar charts on the bottom row. The bar chart on the top right does not clearly depict the temporal smoothness of $f_{3}$, as the amplitude of the wavelet coefficients on $\tau_{a} l_{10}$ for $f_{3}$ is similar to that for $f_{1}$. The reason for the graph wavelet transform not properly capturing the temporal smoothness of $f_{3}$ on $\tau_{a} l_{10}$ is the topology of $G \times H$, which contains many more spatial than temporal edges around $\tau_{a} l_{10}$. In fact, there are only two temporal edges with ends on $\tau_{a} l_{10}$. Therefore, the spatial distribution of $f_{3}$ around $\tau_{a} l_{10}$ impacts more the wavelet coefficients than its temporal distribution. This fact becomes obvious when we remove some spatial edges from the neighborhood of $\tau_{a} l_{10}$, triggering a shift of the coefficients to low frequencies, as illustrated in Figure 25.
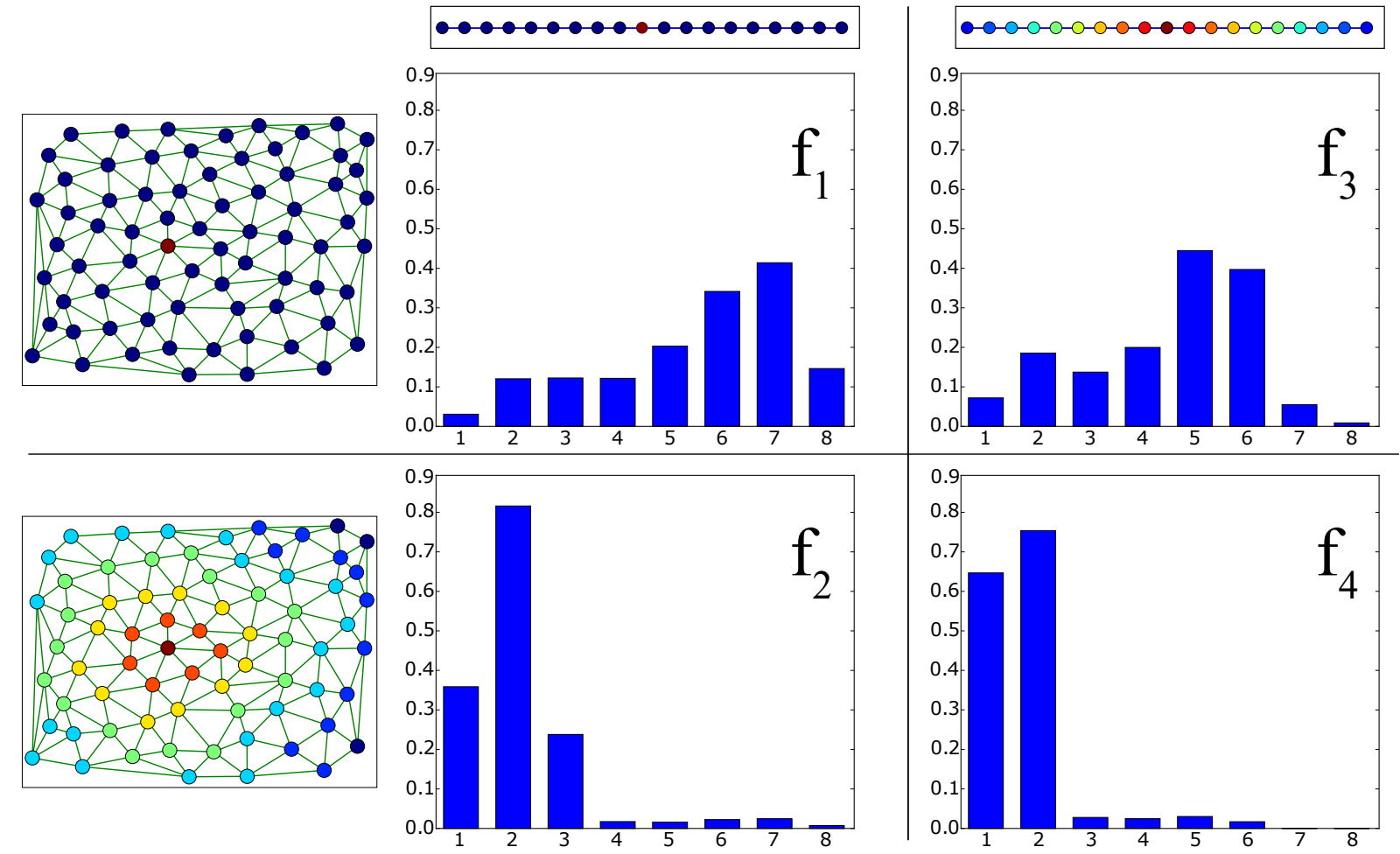

Figure 24 - Wavelet coefficients of node $\tau_{a} l_{10}$ for signals $f_{i}, i=1,2,3,4$. The graph wavelet transform was produced using the SAGW dictionary.

Discussion above raises the question whether it is worth using the Cartesian product graph. Would results be similar if wavelet analysis were applied in each time slice independently? We answer these questions through a real application scenario involving taxi trips in downtown Manhattan. 


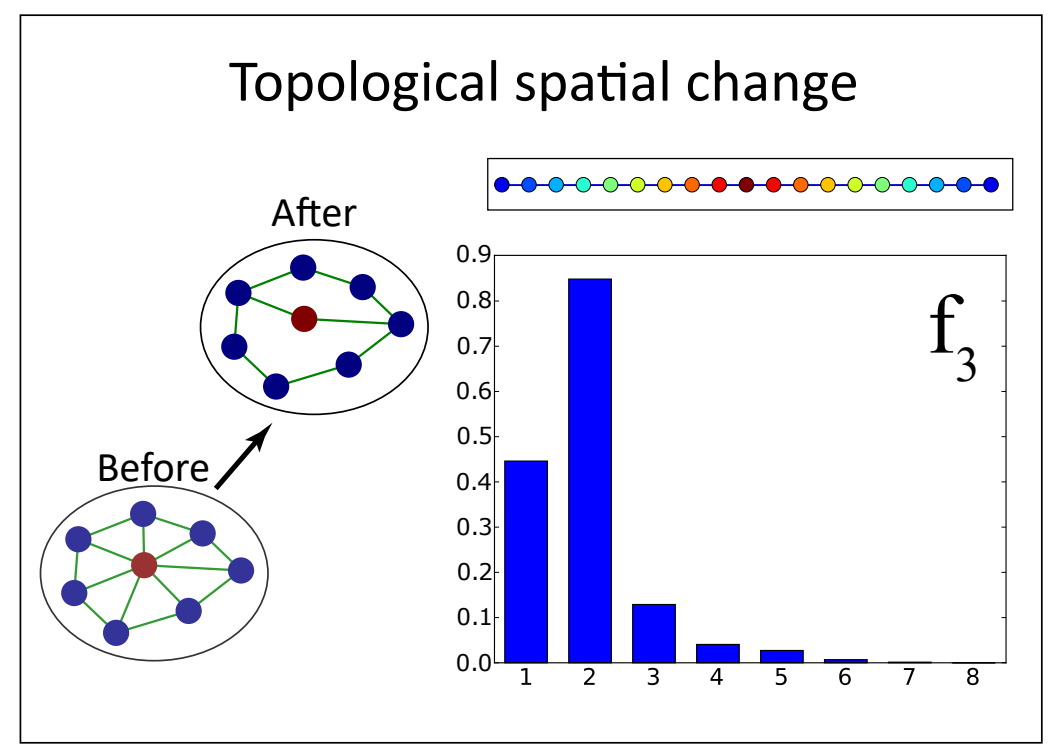

Figure 25 - Wavelet coefficients of node $\tau_{a} l_{10}$ for signal $f_{3}$ after edges removal. The graph wavelet transform was produced using the SAGW dictionary.

Manhattan taxi trips: This example considers real-world data, from NYC TLC $<$ http://www. nyc.gov/html/tlc/html/about/trip_record_data.shtml>, to investigate the addition of temporal edges when analyzing time series defined on the nodes of a graph. The graph corresponds to the street map of downtown Manhattan, with nodes and edges representing corners and streets, respectively. The time series counts the number of taxi pick-ups in the neighborhood of a corner in each time slice.

Figure 26 depicts downtown Manhattan graph with the corners colored by the number of taxi pick-ups on August 12, 2013, 07:30 to 08:00. Zoom-in views in Figure 26 depict the number of taxi pick-ups at and around the corner of 8th Avenue with 41st Street in the consecutive time slices 07:00-07:30, 07:30-08:00, and 08:00-08:30. There is a large spatial variation in the number of pick-ups at the corner of 8th Avenue with 41st Street in all time slices.

By analyzing the (spatial) wavelet coefficients in node 8th-41st at 07:30-08:00 time slice (leftmost column in Figure 27) there is a predominance of mid and high frequencies. On the other hand, the number of taxi pick-ups does not vary drastically among adjacent time slices. Therefore, considering the temporal variation, the signal is smoother. Computing the (spatiotemporal) wavelet coefficients in node 8th-41st at 07:30-08:00 time slice, but considering now the Cartesian product graph, that is, the three time slices are connected by temporal edges, we obtain the distribution of coefficients shown on the leftmost column in Figure 27. The coefficients tend to concentrate in the low frequencies (1 to 3), showing that when the spatial and temporal information are considered simultaneously, the signal tends to be of low frequency. Center and rightmost columns of Figure 27 correspond to the same analysis but considering two other corners, where a shift of the coefficients to low frequencies also takes place when temporal edges are considered. 
From the provided examples, the Cartesian product graph is suitable to handle timevarying data via graph wavelet transform, capturing spatio-temporal properties of the signal under analysis.
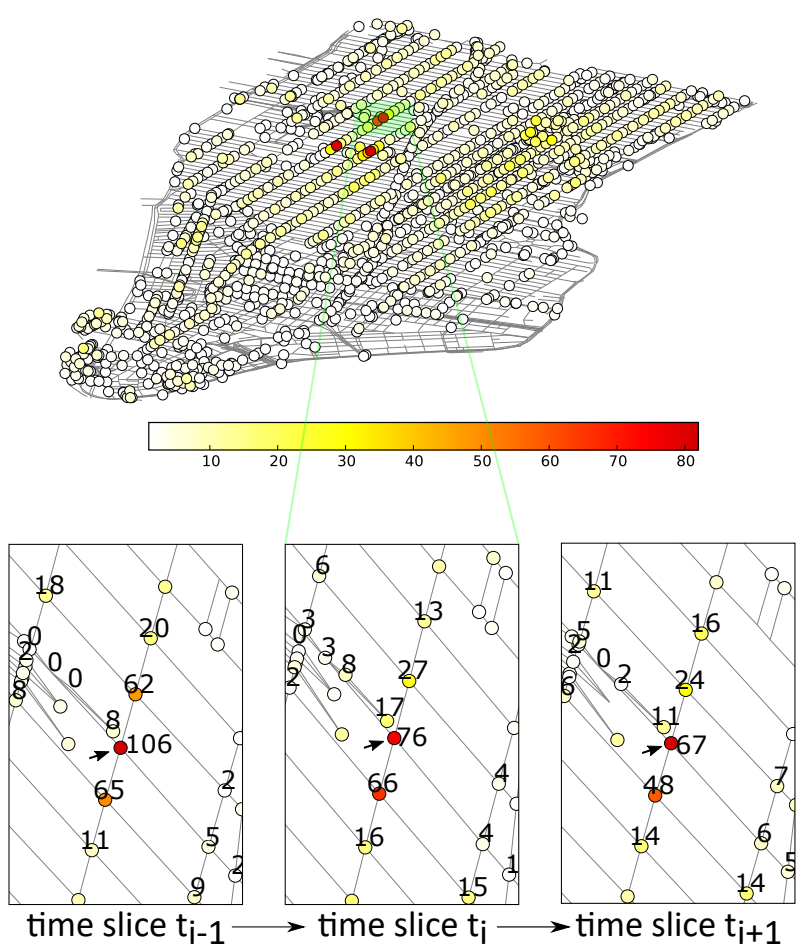

Figure 26 - Number of taxi pick-ups in downtown Manhattan on August 12, 2013, 07:30 to 08:00 (top) and at the corner of 8th Avenue with 41st Street in the consecutive time slices 07:00-07:30, 07:30-08:00, and 08:00-08:30 (bottom). Corner 8th-41st is indicated by arrows in the zoom-in views.
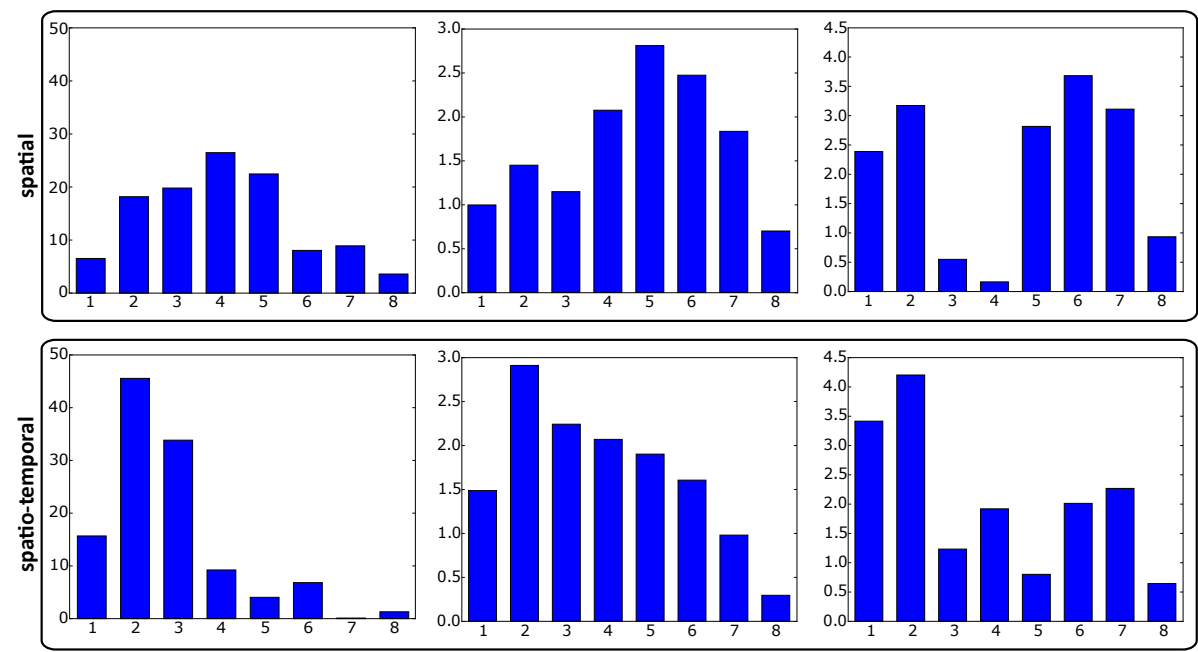

Figure 27 - Spatial and spatio-temporal wavelet coefficients of the nodes on the corner of 8th Avenue with 41st Street (left), Church Street with Duane Street (center), and 6th Avenue with 53rd Street (right) corresponding to August 12, 2013, 07:30 to 08:00. The graph wavelet transform was produced using the SAGW dictionary. 

CHAPTER

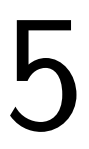

\section{VISUAL ANALYSIS OF DYNAMIC NETWORKS}

This chapter explores data modeled as dynamic network, where the network topology can change over time. The exploration is guided by a signal associated with the nodes of the network, crafted to highlight specific features for the considered application. As further discussed in the following sections, the signal associated with the graph nodes can be obtained in some ways, from attributes naturally present in the nodes to metrics computed from the network topology, providing great flexibility to the proposed method. By analyzing the wavelet coefficients in specific frequencies, our visual analytics method is able to characterize the behavior of the network over time.

The proposed method involves to tackle two main issues: 1) properly define a signal on the nodes of the graph and 2) convert the result of the wavelet analysis in a (set of) time series representations whose behavior reflects phenomena present in the dynamic network. There are nuances involved in the solution of these steps, which we detail in this chapter. Precisely, the problem of properly defining a signal on the nodes of the graph is discussed in Section 5.2 and the problem of converting the output of the wavelet analysis in an enriched time series like representation is detailed in Sections 5.3 and 5.4.

In the graph wavelet theory presented in Chapter 3, the topology of the graph remains unchanged. To adapt that framework to the context of dynamic networks, we need to devise a suitable representation, as described below in Section 5.1. 


\subsection{Dynamic Network Model}

We model a dynamic network as a sequence of $N$ undirected weighted graphs

$$
G_{1}, G_{2}, \ldots, G_{N}
$$

where $N$ is the number of time slices. For each time slice $k \in Z=\{1,2, \ldots, N\}, G_{k}=(V \times$ $\left.\{k\}, E_{k}\right)$ is a graph with nodes $(\tau, k) \in V \times\{k\}$ and edge set $E_{k} \subseteq(V \times\{k\}) \times(V \times\{k\})$. A weight $w_{i j}^{k}$ is associated with each edge in $E_{k}$ connecting nodes $\left(\tau_{i}, k\right)$ and $\left(\tau_{j}, k\right)$. In order to apply the graph wavelet theory, as described in Chapter 3, we add temporal edges between corresponding nodes in adjacent time slices (blue lines in Figure 28), resulting in a graph $G=(V \times Z, E)$ with node set

$$
V \times Z=(V \times\{1\}) \cup(V \times\{2\}) \cup \cdots \cup(V \times\{N\})
$$

and edge set

$$
E=\left(E_{1} \cup E_{2} \cup \cdots \cup E_{N}\right) \cup\left(E_{1,2} \cup E_{2,3} \cup \cdots \cup E_{N-1, N}\right),
$$

where $E_{k, k+1}$ is the set of edges connecting nodes $(\tau, k)$ and $(\tau, k+1)$ in consecutive time slices.

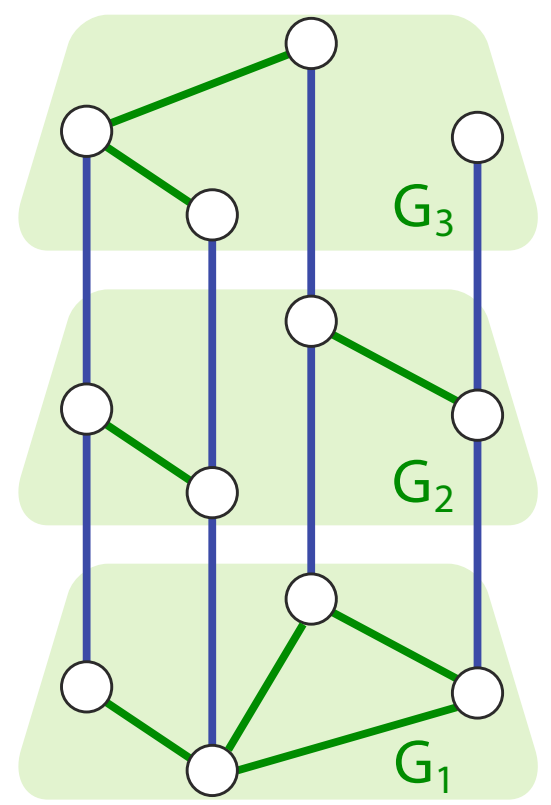

Figure 28 - Dynamic network model.

Since consecutive time slices are connected by temporal edges in $G$, the graph wavelet theory can be applied to analyze a signal defined on the nodes of $G$, allowing the detection of spatio-temporal variations of the signal as well as the identification of important phenomena in the dynamic network as discussed in Section 5.5. Importantly, we use equidistant time-windows 
to aggregate data in time slices. For instance, in one of our usage scenarios in Section 5.5, each time slice corresponds to six minutes of interpersonal contact information.

In a similar model, Valdivia et al. (VALDIVIA et al., 2015) incorporate temporal variation of node attributes in the analysis of static networks. Copies of the same graph $G$ are stacked so that each copy holds the node attributes at a time slice (SANDRYHAILA; MOURA, 2014). Our dynamic network model, in turn, associates a graph $G_{k}$ with each time slice $k \in Z$, allowing variation in both node attributes and network topology.

\subsection{Node Signal \& Edge Weights}

The signal defined on the nodes of a network plays a crucial role in our methodology. Another factor that impacts in our analysis is the weight of the edges, since it dictates how strongly the graph wavelet transform considers each connection between nodes. Larger values correspond to stronger connections, with more impact on the coefficients. An edge weight equal to zero corresponds to an edge that is not present on the graph. The information of the edges is encoded in the graph Laplacian.

The signal to be defined on the nodes can be naturally present in the data, as an intrinsic characteristic of nodes. For instance, one of our usage scenarios in Section 5.5 explores taxi trips in New York City (NYC), where regions of the city correspond to nodes and the signal associated with the nodes is obtained from the number of taxi pick-ups within regions. Edges are placed when the pick-up and drop-off locations take place in different regions/nodes, making the weight of each edge equal to the maximum of the number of trips in each direction. This approach allows the analysis of the variation of node attributes across network structure.

As signal $f$ is a real-valued function, any function can be used. To explore interpersonal contact networks in Section 5.5, we correspond people to graph nodes, with associated signal defined by the number of interpersonal contacts over a period of time. Since each edge represents contact as well, this signal is equivalent to the degree of the node in the corresponding time slice, which is a purely topological measure. This approach enables the analysis of the topological evolution of dynamic networks.

In summary, if the signal takes into account intrinsic characteristics of nodes, one can understand how such property is distributed across the network structure either spatially or temporally. On the other hand, if the signal is computed from topology of the graph, one can explore the dynamics of the network.

Beyond the edges connecting nodes representing different entities, our dynamic network model also includes edges between nodes that represent the same entity in sequential time slices, the temporal edges. The weight of these edges can also be handled to control the effect of temporal variation on the resulting wavelet coefficients. 


\subsection{Node Classification}

Each node $\tau$ of a graph $G$ can be classified using its wavelet coefficient feature vector, a feature vector containing the wavelet coefficients of node $\tau$ ordered from lowest to highest wavelet frequency, that is,

$$
F V_{\tau}=\left(S_{f}(\tau), W_{f}\left(s_{r}, \tau\right), \ldots, W_{f}\left(s_{1}, \tau\right)\right)
$$

Each scale is normalized independently, dividing by the standard deviation and applying a log scale, dividing then by the log of the maximum coefficient. Such transformation brings each coefficient to the range [0,1], while making the classification less sensitive to the amplitudes of the original signal.

Isolated nodes in each time slice are assigned to a specific class, denoted as Zero class and represented by the white color. The other nodes are then separated into five classes using a simple analogy to mechanical torque. Consider a horizontal bar fixed at the middle but allowed to rotate freely. Each coefficient of the feature vector $F V_{\tau}$ corresponds to a force on a specific point of the bar, as illustrated in Figure 29, considering eight wavelet coefficients. Forces acting towards the ends of the bar have greater impact on the torque than those close to the fixed point. The resulting torque indicates whether forces tend to rotate the bar to the left or to the right, that is, whether the feature vector $F V_{\tau}$ has predominantly low or high frequency.

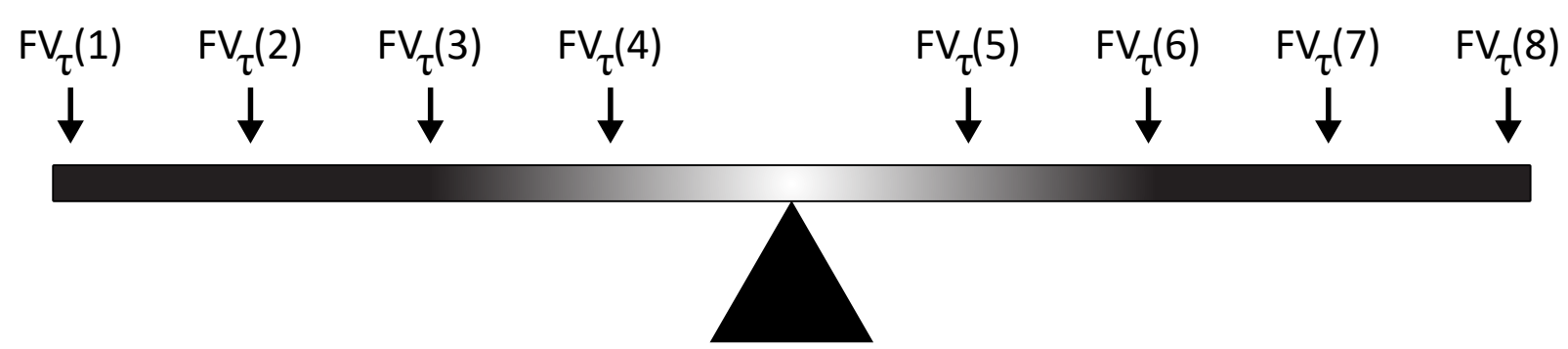

Figure 29 - Torque analogy for node classification. Lowest wavelet frequency at the leftmost end and highest wavelet frequency at the rightmost end.

The torque is then used to classify each node. The torque values are normalized into the interval $[-1,1]$ by simply dividing by the maximum possible torque. Nodes with torque values below -0.3 are classified as low frequency nodes (dark blue), between -0.3 and -0.05 are considered medium-low (light blue), between -0.05 and 0.05 are indefinite (yellow), between 0.05 and 0.3 correspond to medium-high (orange), and above 0.3 are high frequency nodes (red) (Figure 30). Albeit empirically defined, these limits are robust enough to provide reliable results without fine tuning. The classification step is performed using five classes, where the yellow class corresponds to a balance between low and high frequencies. A better discrimination of the intervals $[-1,-0.05]$ and $[0.05,1]$ can be reached considering more classes, however five classes have proved satisfactory results. Moreover, more classes can generate instabilities in 
the classification, since similar nodes can be classified into different classes due to the narrow intervals.

The classification leads to a straightforward interpretation, considerably easier than interpreting the wavelet coefficients directly, where a red node indicates an abrupt change in the signal and a blue node a smooth change in the signal, considering both spatial and temporal neighborhood. Figure 30 illustrates this classification scheme applied to the example from Figure 14. While there are coefficients on the lower frequencies on the feature vector of the middle node, the coefficients are predominantly high frequency, leading to a classification into the red class. For the other nodes, the coefficient on the lowest frequency (the scaling function coefficient) is partially counterbalanced by the coefficients on the higher frequencies, resulting in a classification into the light blue class.

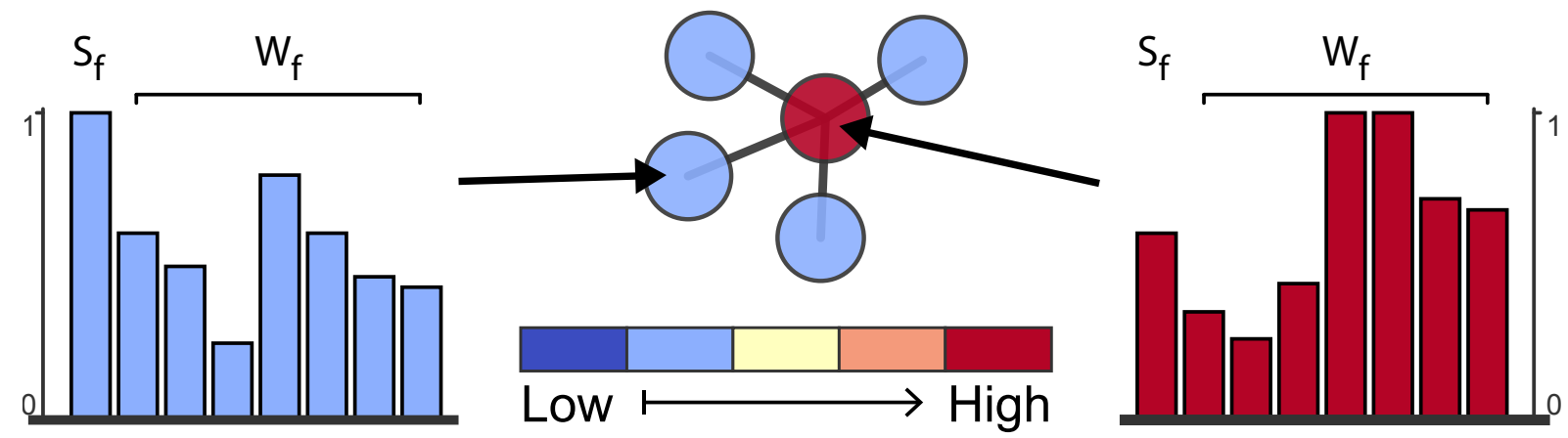

Figure 30 - Node classification performed on the example from Figure 14. The classification represents in a simple manner the information contained in the wavelet coefficients.

\subsection{From Wavelet Coefficients to Visualization}

The proposed visualization aims to provide analytical resources to identify patterns in the dynamics of the network and show how these patterns evolve over time. Specifically, the visualization should enable:

Goal 1. The analysis of the network as a whole. By combining the classification of each node in each time slice, our method can characterize the behavior and temporal evolution of the whole network.

Goal 2. The analysis of each node. The graph wavelet transform reliably identifies local variations in the network. Nodes are classified according to the abruptness of the signal variation.

Goal 3. The identification of prominent nodes. The wavelet coefficients are used to classify the nodes according to signal variations. Nodes classified as high frequency correspond to nodes where the associated signal faces abrupt variations, thus indicating the node behaves discrepantly from its neighbors. The visualization should efficiently guide users to these nodes spatially and temporally. 
Goal 4. The identification of similar nodes. Wavelet analysis enables the identification of nodes with similar behavior over time, regardless of their spatial and temporal location.

The proposed visualization is composed of four linked views: network analyzer, node ranking, node time series, and time slice view, as illustrated in Figure 31.

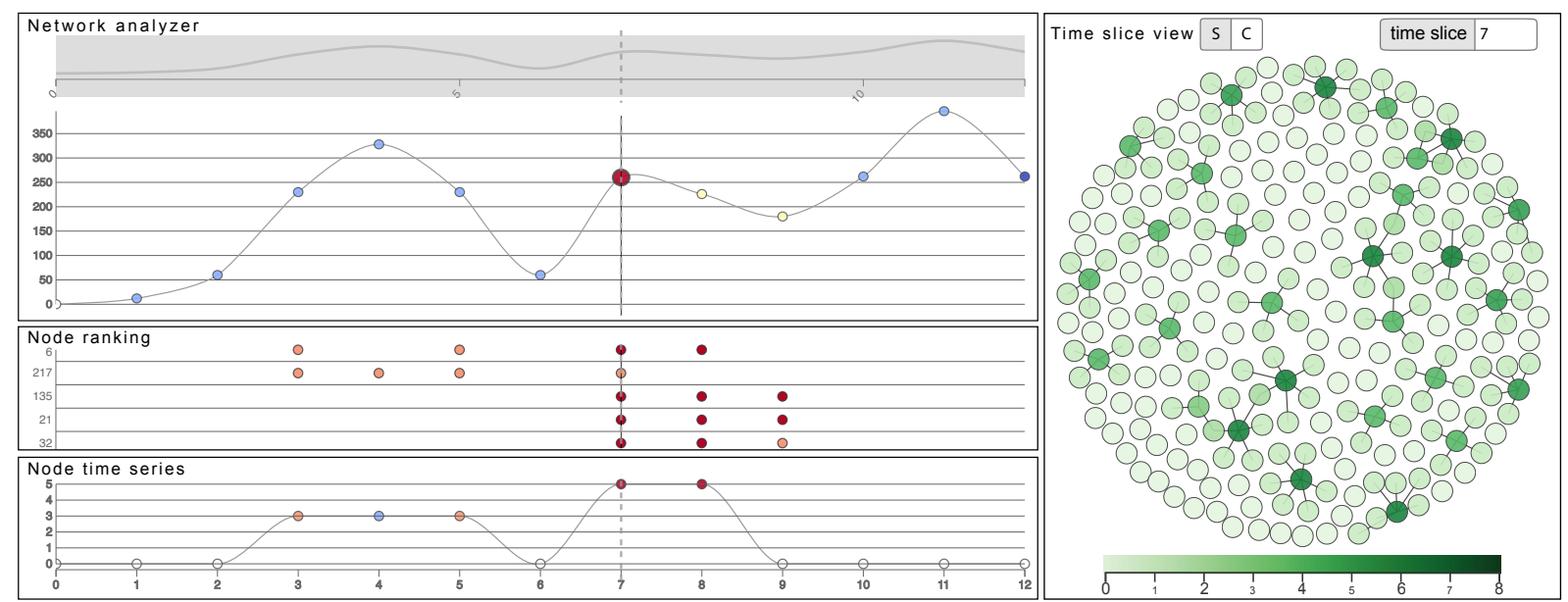

Figure 31 - Proposed interface for dynamic network exploration. It includes four linked components: network analyzer, node ranking, node time series, and time slice view.

Network analyzer: This panel summarizes the general behavior of the network over time via a time series. Each time slice is represented as a circle, whose vertical position is given by the sum of signal of all nodes in that time slice. The coloration of the circle corresponds to the color of the node class with more nodes in that time slice, relative to the maximum number of nodes of that class considering all time slices. This scheme highlights differences in the classification of the network, directing the user to pattern changes. For instance, if in a given time slice the red class has one single node and the light blue class has eight, and the maximum number of nodes in these classes, at all times, is one and ten, respectively, then the circle of this time slice is colored red, to indicate the larger relative presence of red nodes.

Time Slice View: The main motivation of this panel is to visually represent the relationships of a node at a given time slice. The nodes are positioned using Fruchterman-Reingold force-directed algorithm (FRUCHTERMAN; REINGOLD, 1991). The user is allowed to choose between signal and node classification visualizations. By positioning the mouse over a node its contact partners and edges connecting them are highlighted. Further, additional information is revealed, such as node identification and class.

Synthetic Dynamic Network Exploration: To properly demonstrate the capabilities of our method, we generated a synthetic dynamic network containing 250 nodes and 13 time slices. The signal associated with a node $(\tau, k)$ is the number of edges in $G_{k}$ incident on the node. The weights of the edges are all equal to one. There are two large spatial events (leftmost panel in Figure 32 ) in the network, corresponding to an activity peak (many edges) at time slice 4, which decrease in size until time slice 6. At time slice 7, several small spatial events (central panel 
in Figure 32) appear, with some of them disappearing on the next time slices. At time slice 10, another two large spatial events (rightmost panel in Figure 32) are created, which reach an activity peak at time slice 11 and decrease at time slice 12 .

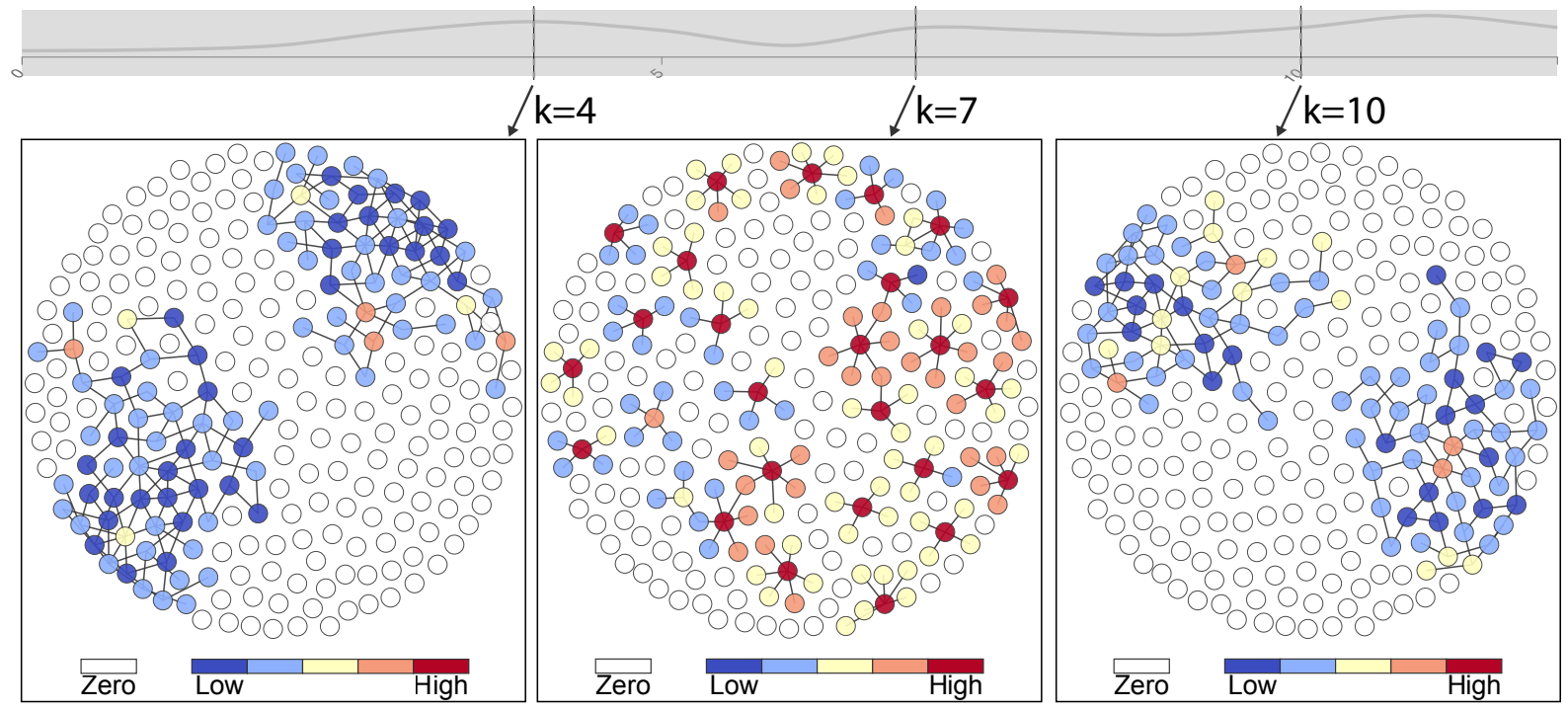

Figure 32 - Node classification for the synthetic dynamic network in time slices 4, 7, and 10. All these time slices have similar heights on the network analyzer. However, time slices 4 and 10 are classified as light blue, while time slice 7 is classified as red.

Figure 31 depicts the network analyzer for the synthetic dynamic network. It shows that the activity level (number of edges) of the synthetic dynamic network increases and decreases, with three peaks, at time slices 4, 7, and 11. Most time slices are classified as light blue, which indicates the predominance of nodes facing smooth signal variation. A high concentration of this kind of node suggests a large event happening on the dynamic network, which is the case for time slices 4 and 10, as illustrated in the leftmost and rightmost panels of Figure 32. Time slice 10 has height similar to time slice 7 in the network analyzer, indicating similar activity level, but these two time slices differ in their classification. In fact, time slice 7 is classified as red and time slice 10 is classified as light blue. This change in the classification indicates a difference in the current behavior of the network, albeit with almost the same level of activity. A red time slice indicates the predominance of high frequency nodes. Therefore time slice 7 contains nodes facing abrupt signal variation, revealing small events probably with central nodes since the signal corresponds to the degree of the nodes. As illustrated in the middle panel of Figure 32, this is indeed the case, corresponding to several small events that suddenly appeared at time slice 7 .

By considering the position (height) and color of the circles, we can infer consistent states of the network, visually identifying instants with similar configuration and signal distribution. The capability of identifying time slices with similar network activity level while discriminating patterns of behavior is one of the traits that differentiates our approach from others in the literature, as for example the method proposed by Elzen et al. (ELZEN et al., 2016), called Reducing Snapshots to Points (RSP), where similar states are also visually grouped, but not discriminated. As we shall see in more detail in Section 5.5, our method not only visually 
identifies similar states, but also provides additional information about them. Indeed, users gain insights about the general behavior of the network at each time slice without directly inspecting each of the time slices.

Figure 33a illustrates the RSP method applied to the synthetic network. The circles corresponding to time slices 4 and 10 are projected distant from each other, indicating different network configurations. From the viewpoint of our method, these times are similar, almost the same activity level and two large events. The RPS method differentiates them because it takes into account which nodes are involved. At a glance, our network analyzer revels all these information, while the RSP method requires additional views to bring meaning to the difference visually asserted.

In addition, we use another state-of-the-art technique, called TimeArcs (DANG; PENDAR; FORBES, 2016), to explore the synthetic dynamic network. The TimeArcs method also reveals different nodes involved in two major events in time slices 4 and 10, as shown in Figure $33 \mathrm{~b}$. However, the activity pattern presented in the middle panel of Figure 32, namely small events with central elements, is not easily perceived, while our tool makes it clear in advance thanks to the network analyzer.

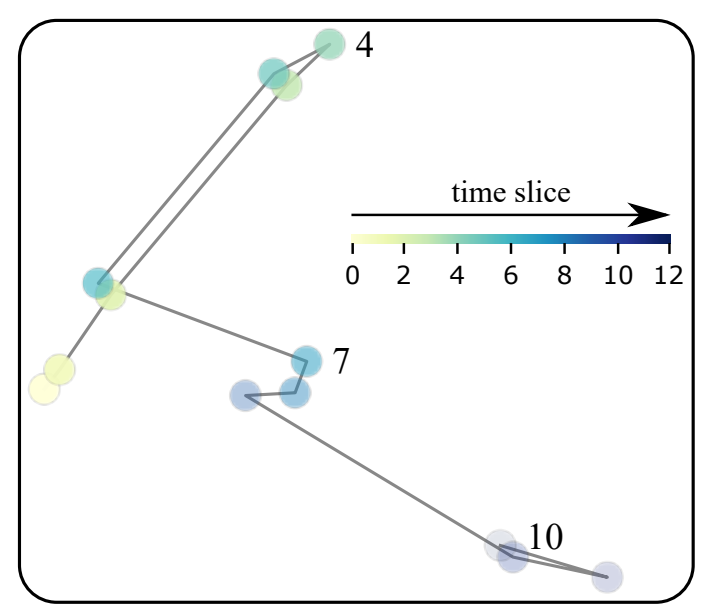

(a) RSP

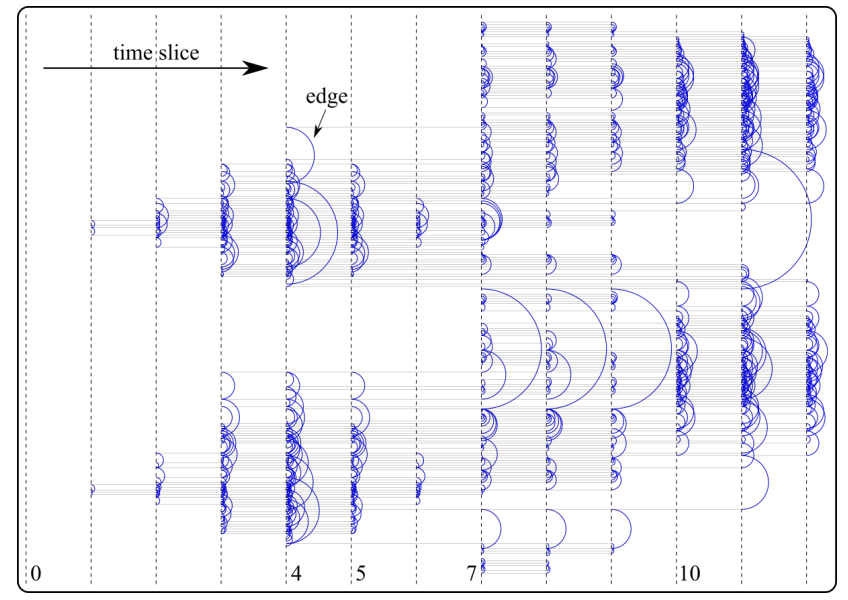

(b) TimeArcs

Figure 33 - RSP and TimeArcs methods applied to the synthetic dynamic network.

Node ranking: This panel depicts the nodes ordered according to their relevance. Since relevance is application dependent, we adopted a simple mechanism to rank nodes, where relevance is related to the higher frequencies. Therefore, we consider a node as relevant in a time slice if it is classified as orange or red, and we rank a node according to the total number of time slices in which the node is considered relevant, on a given time frame. Considering the whole synthetic dynamic network, the most relevant entity corresponds to the node identified by the number six, which was classified as orange/red in four time slices (middle left of Figure 31). Indeed, this node was involved in one of the large events in the early time slices, near the border of the event, which led to a higher frequency, and was the center of a small event later. 
By clicking on a node in the node ranking, the order changes to organize the nodes according to their similarity to the selected node. Similarity is defined as the intersection between the two corresponding relevance arrays, displayed in the node ranking. Considering the synthetic dynamic network, this option would quickly reveal nodes similar to node six, namely, nodes that were center of small events.

Since this definition of similarity is the number of time slices where two entities are classified as relevant, which is not related to the activity curves of the entities, the results may seem counterintuitive. This measure identifies entities that had significant change at the same times, regardless of the overall behavior or format of the activity curves.

This panel acts as a visual index of the relevant nodes, allowing users to quickly identify nodes and time slices of most relevant changes. This is a new and important feature for the visualization of dynamic networks, bridging the gap between exploring the network as a whole and specific nodes, reducing the amount of direct inspections required to find relevant information.

Node time series: Once an entity is selected in one of the panels, the corresponding time series is depicted below the node ranking panel. The height of the circles represents the signal and the coloration indicates the node classification in each time slice, as illustrated on the bottom left of Figure 31.

Figure 31 depicts the node time series of node six in the synthetic dynamic network. The time series indicates two periods of activity, from time slice 3 to 5 and from time slice 7 to 8 . The first one may seem curious to the reader, since the node has the same number of contacts with different classifications, but the pattern hidden in the first period reveals the strength of the graph wavelets. Indeed, Figure 34 shows the neighborhood of node six in time slices 3 and 4 (the contacts in the neighborhood of node six is identical in time slices 3 and 5). The way node six interacts with its neighbors does not change over time, however some neighbors of this node expand their contact beyond node six, making node six a member of a slightly larger contact network and no longer a central node of a small group.

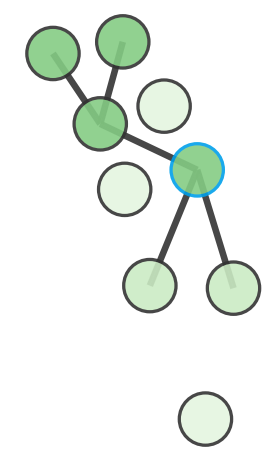

(a) Time slice 3

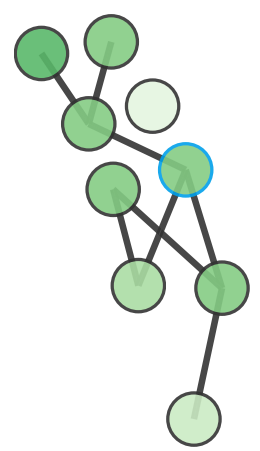

(b) Time slice 4

Figure 34 - Neighborhood of node six (highlighted node) in the synthetic dynamic network. Only nodes up to order two are shown. Although some edges of second-order nodes were omitted, they contribute to the signal of these node. 
The panels comprising our visual analytics tool are interactive and linked, allowing users to explore the information in several ways. The network analyzer guides the user through the temporal evolution of the network (Goal 1), including consistent network states. The node time series and time slice view, combined, allow for the exploration of the signal and classification of each node (Goal 2). The node ranking panel summarizes which entities are more prominent (Goal 3), and allows for the identification of entities with similar patterns of relevance over time (Goal 4).

Further, the use of graph wavelet transform, enabled by these panels, creates a visualization tool whose performance surpasses the current state-of-the-art, allowing users to explore and discover gist information and patterns efficiently, with less interaction and mental effort.

\subsection{Usage Scenarios}

To demonstrate the capabilities of the proposed method, we consider two distinct real dynamic networks. The first dataset contains human interaction information over time, while the second contains georeferenced information about taxi trips in NYC. The prototype visualization interface was implemented in JavaScript. A Python script converts the information from its original format into the dynamic network model presented in Section 5.1, calculates the signal and edge weights, performs the approximation of the wavelet coefficients and node classification, and saves the results in a format suitable for the visualization interface. The interface, source codes, and additional applications are available on a GitHub page located at $<$ https://github.com/ AlcebiadesDalColJunior/>.

High School Dynamic Contact Network: The high school dataset (FOURNET; BARRAT, 2014) contains face-to-face contact information between 180 students from a school in France, during nine days in November of $2012<$ http://www.sociopatterns.org > . Each student belongs to one of five different classes. The original data provides contact information between students in intervals of 20 seconds, which we aggregated further by creating a time slice every 6 minutes, for a total of 2,027 time slices. The resulting dynamic network has 14,788 edges, not counting the temporal edges. The signal associated with each node is the number of face-to-face contacts made by the corresponding person in the corresponding time slice, and the weight of each edge corresponds to the number of face-to-face contacts between the two involved people. The approximation of the wavelet coefficients took approximately 7 seconds, and the whole preprocessing step took approximately 20 seconds on a regular i7 computer, using around $1 \mathrm{~Gb}$ of memory.

We start the exploration of the high school dynamic network using the network analyzer, which shows the total number of contacts made by students (Figure 35). As expected, there are seven periods of activity, corresponding to the seven weekdays where the students interacted. Interestingly, Wednesday has a much lower level of activity than the other weekdays, which 
indicates a lower level of interactions between the subjects. Indeed, on this day, the students had exams, which lessen the level of interaction (FOURNET; BARRAT, 2014; ELZEN et al., 2016).

The predominance of light blue nodes indicates that in most of the time slices the signal faces smooth variation, meaning that children interact primarily in balanced contact groups. This dataset is naturally biased to low frequencies, as no node was classified as red. Some time slices, however, they are classified as orange, mostly on Monday, suggesting sudden changes in the number of contacts and directing our attention to a pattern different from the one mentioned above. The network analyzer allows for zooming particular time-intervals, in particular the zoomed-in view at the bottom in Figure 35 shows a view of activities on the first Monday. To demonstrate the usefulness of the classification scheme used in the network analyzer, we selected, in the zoomed Monday, three time slices with different classification, corresponding to times 9:24 (orange), 10:24 (yellow), and 14:30 (light blue), illustrated in Figure 36.

The 9:24 time slice is the first to be classified as orange on the dynamic network. From the network analyzer only, one can infer that this time slice does not contain much activity, the height of the corresponding circle is well below the peak for this day. The 9:24 time slice is part of a consistent level of activity that covers the morning period. While most of the neighboring time slices are classified as light or dark blue, the 9:24 time slice was classified as orange. This classification implies a difference in the contact pattern, but not abrupt enough to be considered red. Since the graph nodes are predominantly low frequency, the presence of a few higher frequency nodes would be sufficient to lead the time slice to a higher frequency classification.

By inspecting the 9:24 time slice, as illustrated in Figure 36 (leftmost column), it is clear that this time slice mostly contains pairs of contacts, with low signal (number of contacts). The nodes are mostly light blue with a few yellows. However, three connected nodes on the leftmost student class present a higher signal, leading to a orange classification, which leads to the classification of this time slice as orange as well. In more practical terms, this phenomenon probably represents a transmission of a large amount of information, where the center node

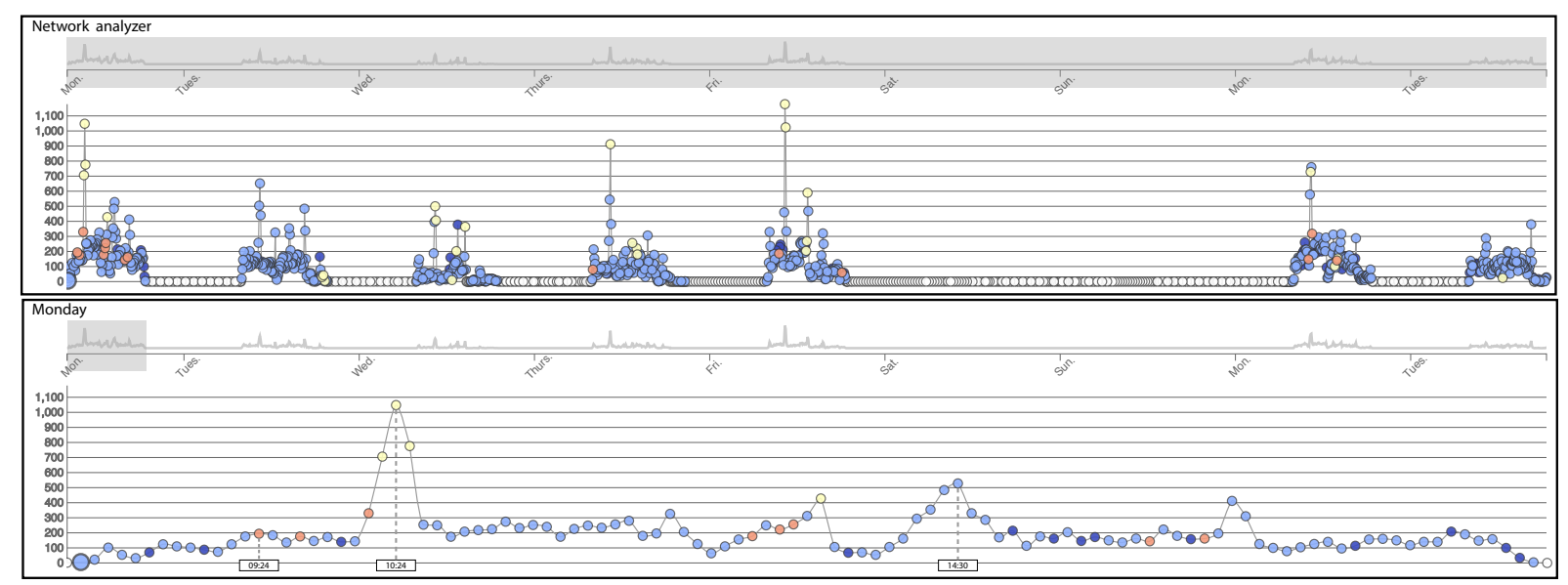

Figure 35 - Network analyzer for the high school dataset. The panels depict the whole dataset (top) and a zoomed-in view of the first Monday (bottom). 
corresponds to the child who transmitted the information. In other words, this activity peak is different of the pattern of behavior at this time. This change is correctly detected and represented by the network analyzer, reducing the amount of direct inspection and comparisons with respect to other methods in the literature for dynamic network analytics.

The activity peak for Monday happened at 10:24, where the sum of the signal surpassed a thousand. Since the sum of the signal corresponds to the total number of contacts and the time slice is classified as yellow, this time slice contains a large event, with some moderated signal differences. Indeed, more pronounced signal differences and/or smaller events would cause a number of the nodes to be classified into a higher frequency class, which would classify the time slice into a higher frequency class as well. Similarly, a large event without somewhat significant signal changes would be predominantly light or dark blue. In other words, this time slice contains nodes with moderate signal connected to several nodes with lower signal, leading to a classification as yellow. As illustrated in Figure 36 (middle column), this is clearly the case. This time slice includes interactions between four of the five classes of students.

The 14:30 time slice also contains a considerable amount of activity, indicated by the height in the network analyzer, and beyond that this time slice is classified as light blue. The combination of increased height and light blue classification means that this time slice contains a large event, without signal peaks. Smaller events or peaks would lead to higher frequencies, as

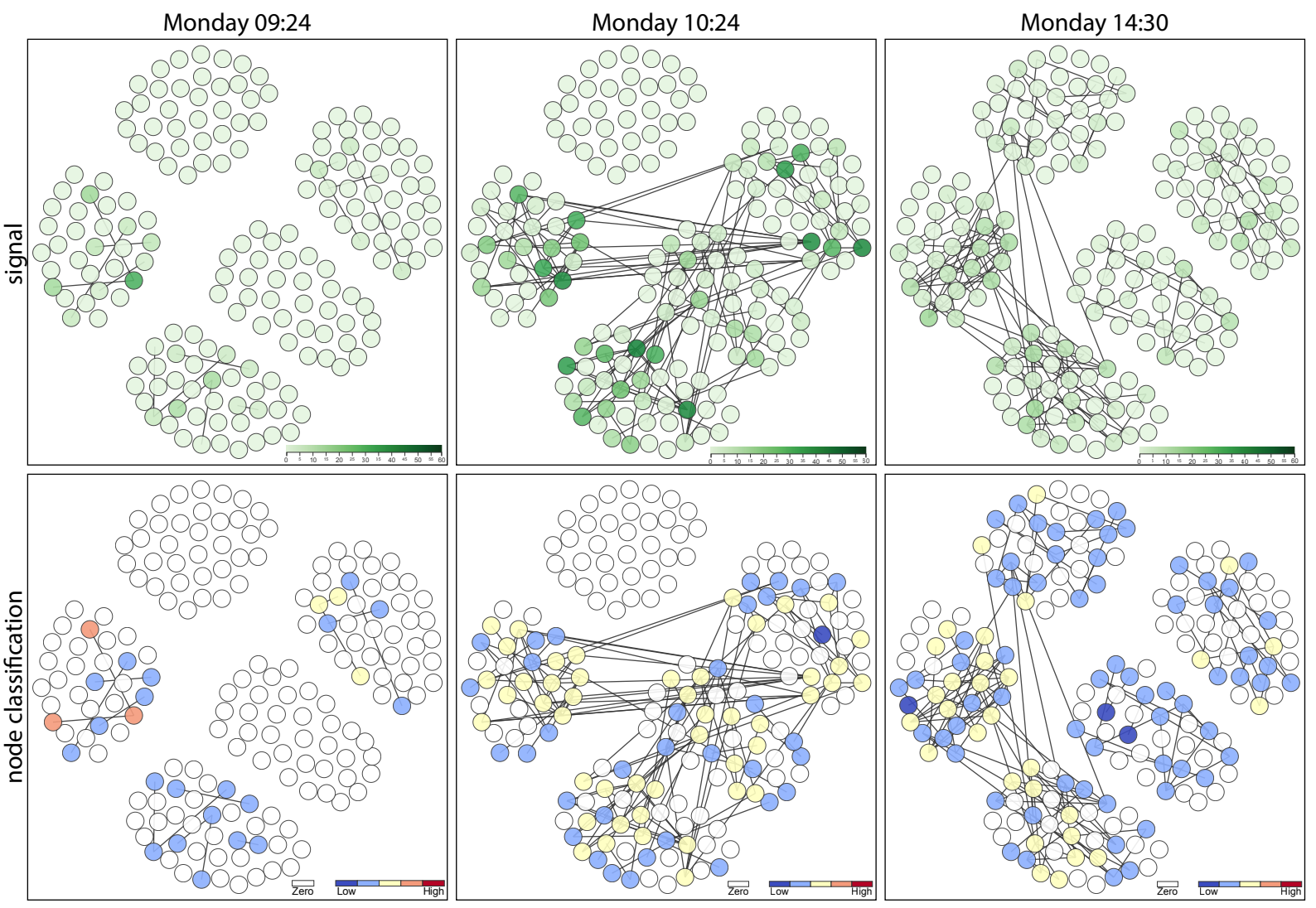

Figure 36 - Signal and node classification for the high school dataset on the first Monday at 9:24, 10:24, and 14:30. 
was the case for the 10:24 time slice. Indeed, as illustrated in Figure 36 (rightmost column), the signal varies smoothly across a large event, with some nodes classified as yellow, but with the relative majority of light blue nodes. This large event is harder to notice when compared to the synthetic dynamic network where we had a controlled environment, highlighting the potential of the graph wavelet transform in the exploration of dynamic networks with the aid of visual analysis.

These analyses highlight the potential of the network analyzer, illustrating that it represents relevant information concerning the signal under analysis and topology of the network, greatly reducing the necessity for inspection of individual time slices. While the proposed interface allows for closer inspection of each time slice and node, the network analyzer efficiently provides overall information about the evolution of the dynamic network.

The main idea behind our network analyzer - to present a simple summary of the temporal evolution of the network to guide the user through the exploration - is not entirely new. In fact, our work was inspired by Reducing Snapshots to Points (RSP) (ELZEN et al., 2016). However, the use of the graph wavelet theory allows our method to leverage more information efficiently. The high school dataset was chosen exactly because it was used as a case study in RSP paper. We implement RSP following all guidelines given by the original paper and apply it to the high school dataset. Figure 37 depicts a direct comparison against the RSP method.

Importantly, the RSP method considered a different aggregation of edges, using 6 minutes intervals with an overlap window of 60 minutes, centered at the considered time, while our method uses only 6 minutes intervals. Since this aggregation can be seen as a low-pass filter, running our method with overlapping windows results in forcibly lower frequencies. Conversely, if RSP is applied to data without an overlap window, the paths corresponding to each day do not appear, because each time slice is considerably different from the previous. Therefore, Figure 37 shows the best results of both methods. We consider that a smaller temporal aggregation is beneficial, since more details can be revealed, thus better depicting the evolution of the network.

From Figure 37, one can see that for our method both the position and color of each circle have a clear meaning, while for RSP, the color represents time and the position is derived from dimensionality reduction techniques, which cannot be as easily interpreted, especially when using non-linear methods. While there are other possible layouts for RSP, the main insight available for the user is that there are consistent states, where time slices are grouped at an arbitrary position. Our method represents these states and provides information regarding the activity and general behavior of the network at each time slice.

Specifically, consider the three time slices indicated in Figure 37. While RSP's layout correctly separated the three points, it is not clear how these time slices are different or even the temporal relationship between them. Our method naturally places these points along the time axis, representing the activity level and the information about the time slice. Contrarily to RSP, using our network analyzer, the user can obtain valuable information, without inspecting the 

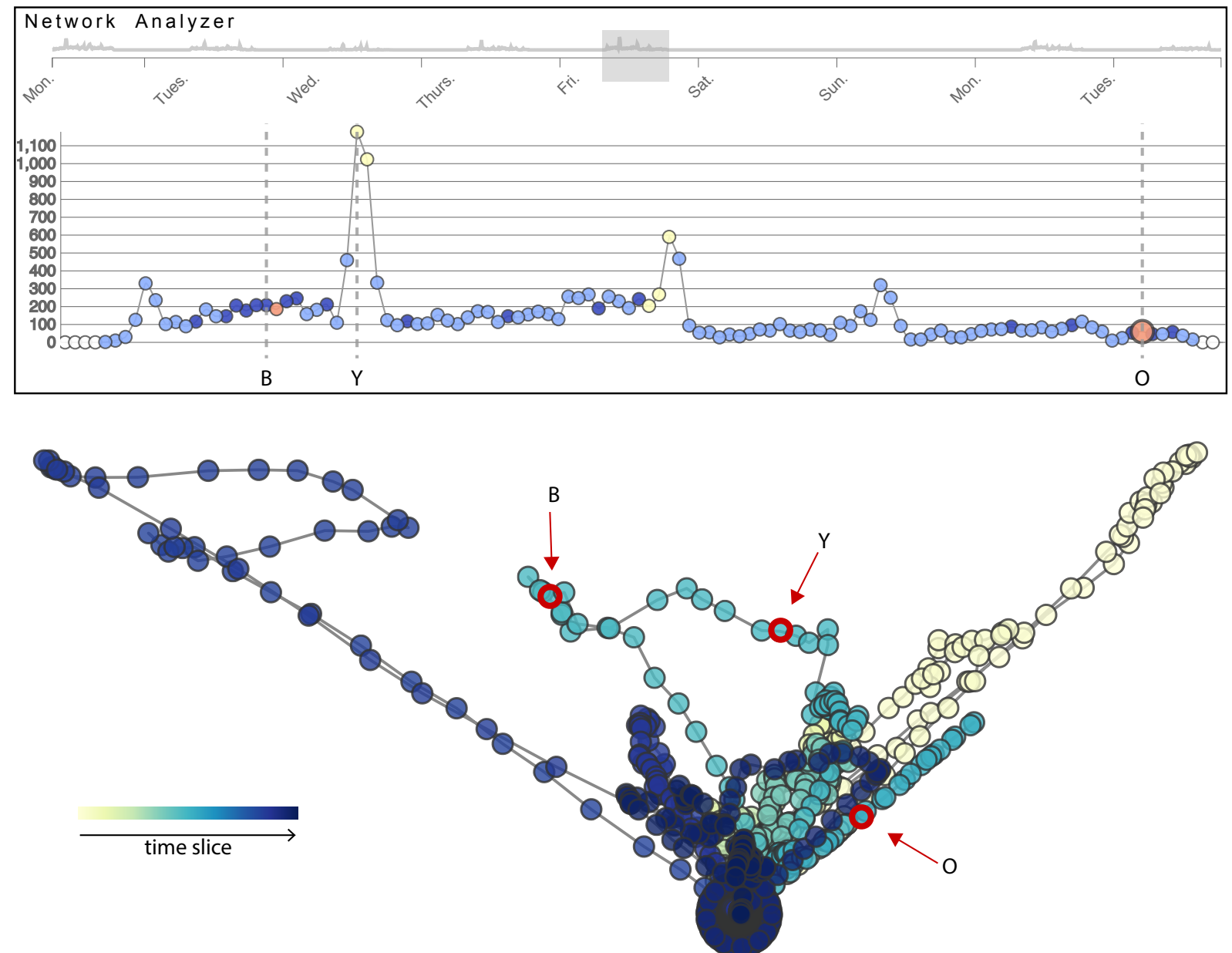

Figure 37 - Comparison against the RSP method considering the high school dataset. Our network analyzer, focusing on Friday (top). Projection view of the RSP method (bottom); circles are colored to encode the time, darker markers correspond to older snapshots. The letters $B, Y$, and $O$ create a temporal match between the views.

corresponding time slices. For instance, the level of activity at time $Y$ is higher than at times $B$ and $O$, time $O$ contains nodes with moderate signal variation, and so on.

Moreover, while the RSP method is used to explore only the topology of the dynamic network, our method can be used to simultaneously explore the topology and any other signal defined on the graph. Indeed, the wavelet analysis applied to the same network can yield different results by changing the associated signal. This enables the exploration of different facets of the data, which is not easily done in other methods from the literature.

To further relate our method with the state-of-the-art, we apply the TimeArcs (DANG; PENDAR; FORBES, 2016) method to the high school dataset by using the source scripts made available by the authors. Figure 38 depicts the students as horizontal lines and their interpersonal contacts as blue arcs. Although the method vertically group nodes according to similarity, there is a large amount of edge cluttering. Further, long arcs interfere with the visualization of other time slices, they come from student pairs that have low interpersonal contact along all time frame. 


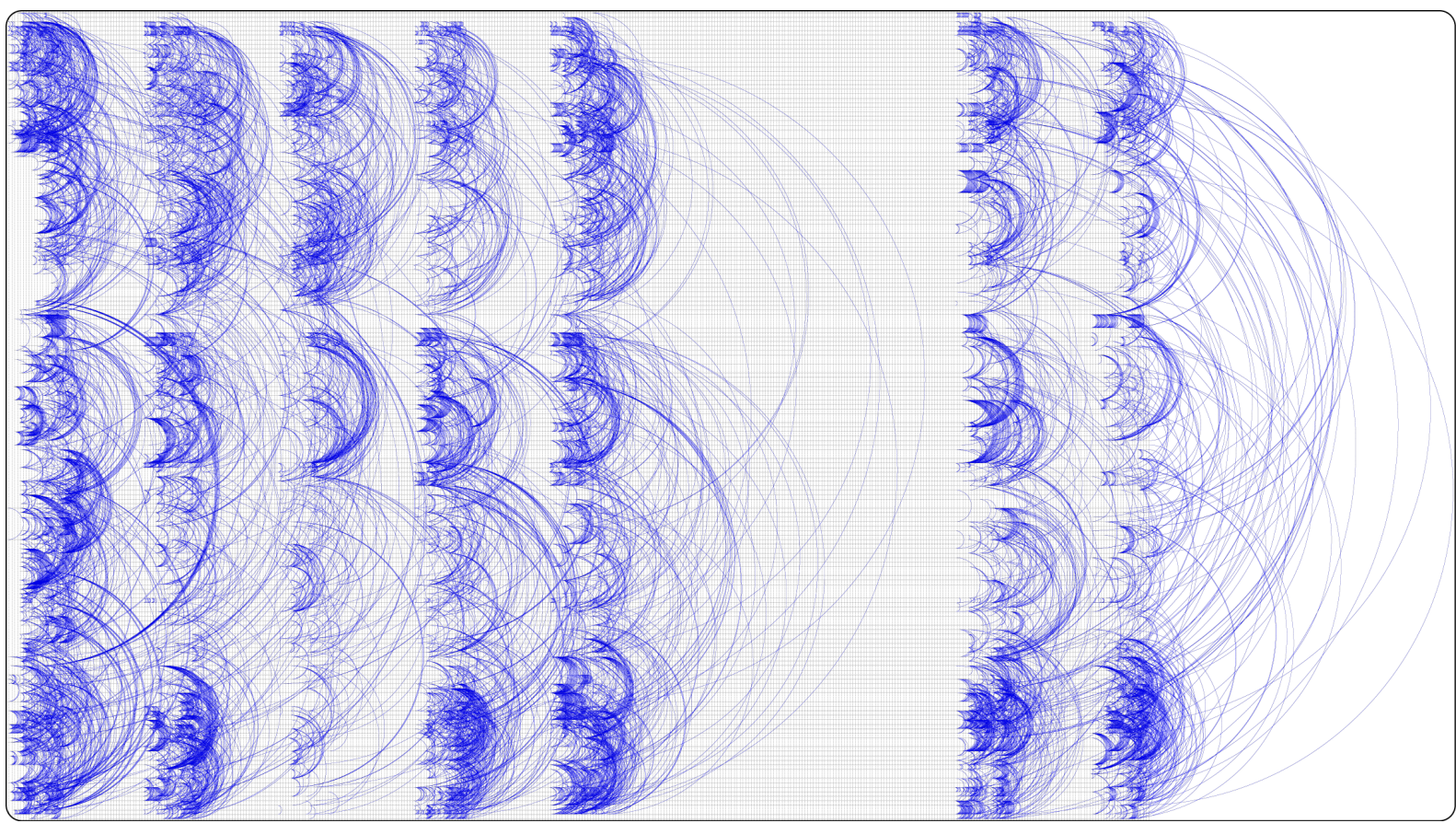

Figure 38 - TimeArcs method applied to the high school dataset. The temporal evolution is represented along the $x$ axis.

Manhattan Taxi Pick-Ups: The Manhattan taxi dataset contains taxi trips in Manhattan in December 2009, created from NYC Taxi and Limousine Commission (TLC) Trip Record Data $<$ http://www.nyc.gov/html/tlc/html/about/trip_record_data.shtml>. Each taxi zone is defined by TLC and corresponds to a node, with the associated signal corresponding to the number of taxi pick-ups on that zone. We restricted our attention to the zones in Manhattan and the three nearby airports. Edges are placed between two zones if there are trips between them, and the weight of each edge is the maximum of the number of trips in each direction between the two involved zones. Each time slice corresponds to 10 minutes, for a total of 4,464 time slices. The resulting dynamic network has 5,078,722 edges, not counting the temporal edges. The preprocessing time was around 40 seconds, with the approximation of coefficients taking about 5 seconds, using about $3.7 \mathrm{~Gb}$ of memory.

Figure 39 illustrates the proposed visual analytics interface on the Manhattan taxi dataset. The network analyzer shows that the nodes/zones have predominantly light blue classification, indicating smooth taxi pick-ups distributions. However, there are several time slices with red classification, in a periodic pattern, which means a significant difference in the number of taxi pick-ups with respect to neighboring time slices and zones (Goal 1). By selecting the first one, corresponding to December 5th at 02:00, the time slice view changes to the respective time, showing two nodes with red classification, corresponding to Lower East Side and East Village (Goal 2).

By selecting these zones, we can inspect the corresponding time series, illustrated in Figure 40, which confirms that this is a periodic occurrence on the first hours of weekends (Goal 


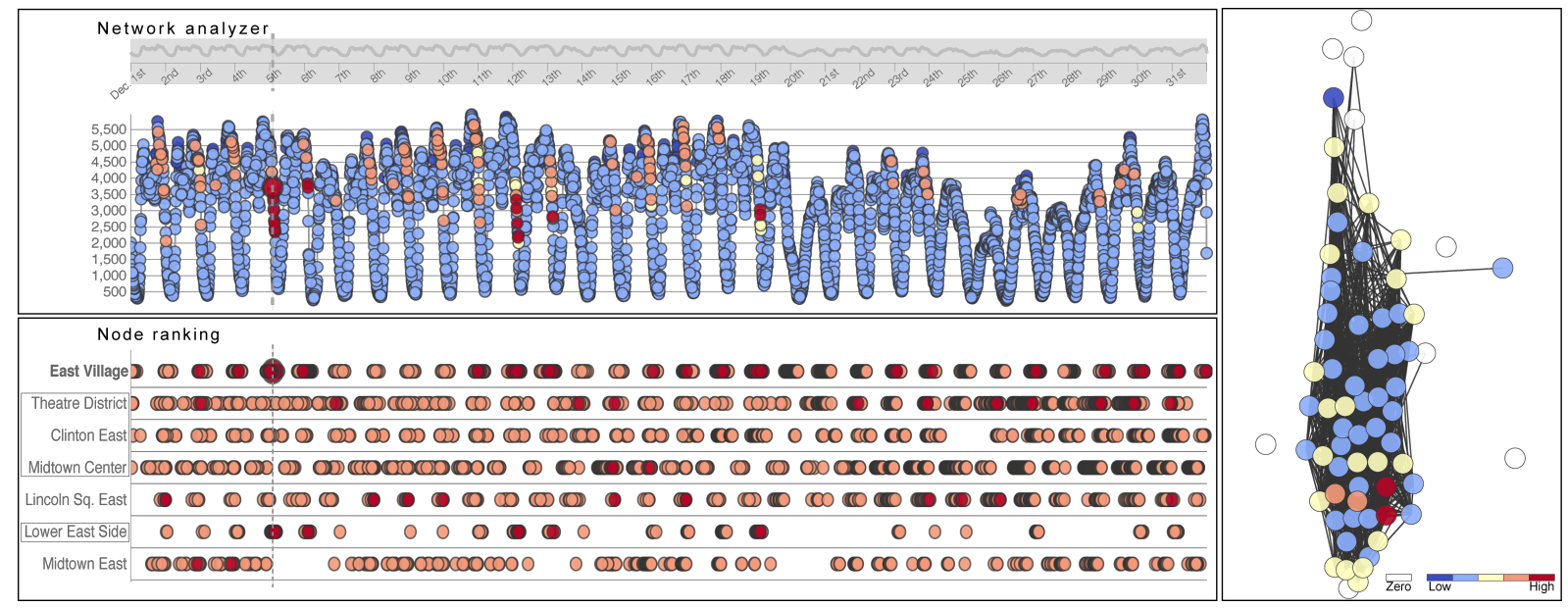

Figure 39 - Interface considering taxi trips in Manhattan in December 2009. The network analyzer depicts the total number of taxi pick-ups. The nodes in the time slice view (right) represent taxi zones and they are connected in case of trips between the corresponding zones. The node ranking was adjusted to order zones according to their similarity to East Village.

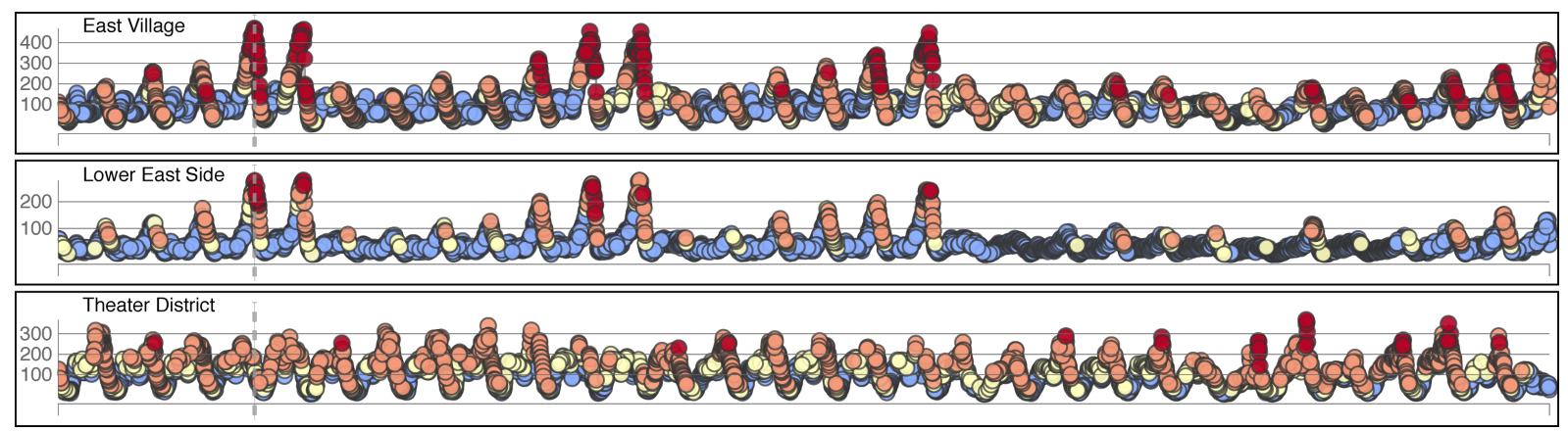

Figure 40 - Node time series of East Village, Lower East Side, and Theater District zones. The vertical dotted lines correspond to December 5th at 02:00.

2). Since the East Village is known for its vibrant nightlife, this is indeed expected. By changing the ranking order to similarity to East Village (selecting the East Village node in the node ranking panel), we can explore which zones have similar temporal relevance patterns (Goal 4), which include the Theater District, Clinton East, and Midtown Center.

The most similar region to East Village, in terms of relevance of nodes, is Theater District, which has a different temporal activity pattern, as illustrated in Figure 40. Indeed, the activity for this region is somewhat constant, potentially due to the Port Authority Bus Terminal and the attractions of the region, particularly in this time of the year. Because the Theater District is classified as relevant for most of the time slices, there is significant overlap of relevant time slices between those two regions, which leads to the high similarity value in the node ranking panel. Clinton East and Midtown Center also have a similar behavior, but with different distributions of high frequency among time slices. Lower East Side, while more distant in the ranking, is classified as high frequency on approximately the same time slices as East Village. Indeed, the corresponding time series presents a similar activity pattern, with significant peaks on the first hours of weekends. However, this region has less overlaps of relevant time slices, because of 
less activity on the start of the week and during the holidays, which reduced the similarity value between them.

Back to the network analyzer, the number of taxi pick-ups for the whole city was reduced by the holidays, which is expected. The daily patterns are clearly visible, with Sundays having less taxi activity than the weekdays, and December 25th was the day with lowest activity of the month. However, there is a small gap around December 20th, which corresponds to a blizzard that arrived in NYC around 13:30. The afternoon peak in taxi trips, that usually occurred around 20:00 on the nearby days, happened at 19:00 on December 19th, and the morning of the next day, a Sunday, also shows a slower increase in the number of trips, compared to other Sundays of this month. Interestingly, this whole period is classified as light or dark blue, indicating that the patterns of taxi pick-ups were similar over most of the city.

Figure 41 illustrates the proposed interface considering the period between December 24th and December 27th. The most relevant region for this time period is the Theater District (Goal 3), with three pronounced peaks of activity, two red periods in December 25th and 26th, both at 22:30, and one orange in December 25th at 18:30. While the exact reason for these significant peaks is unknown, we conjecture that it can be caused by people returning to the city from nearby locations, on a day with reduced options for transportation, possibly combined with tourists leaving the holiday attractions in the area.
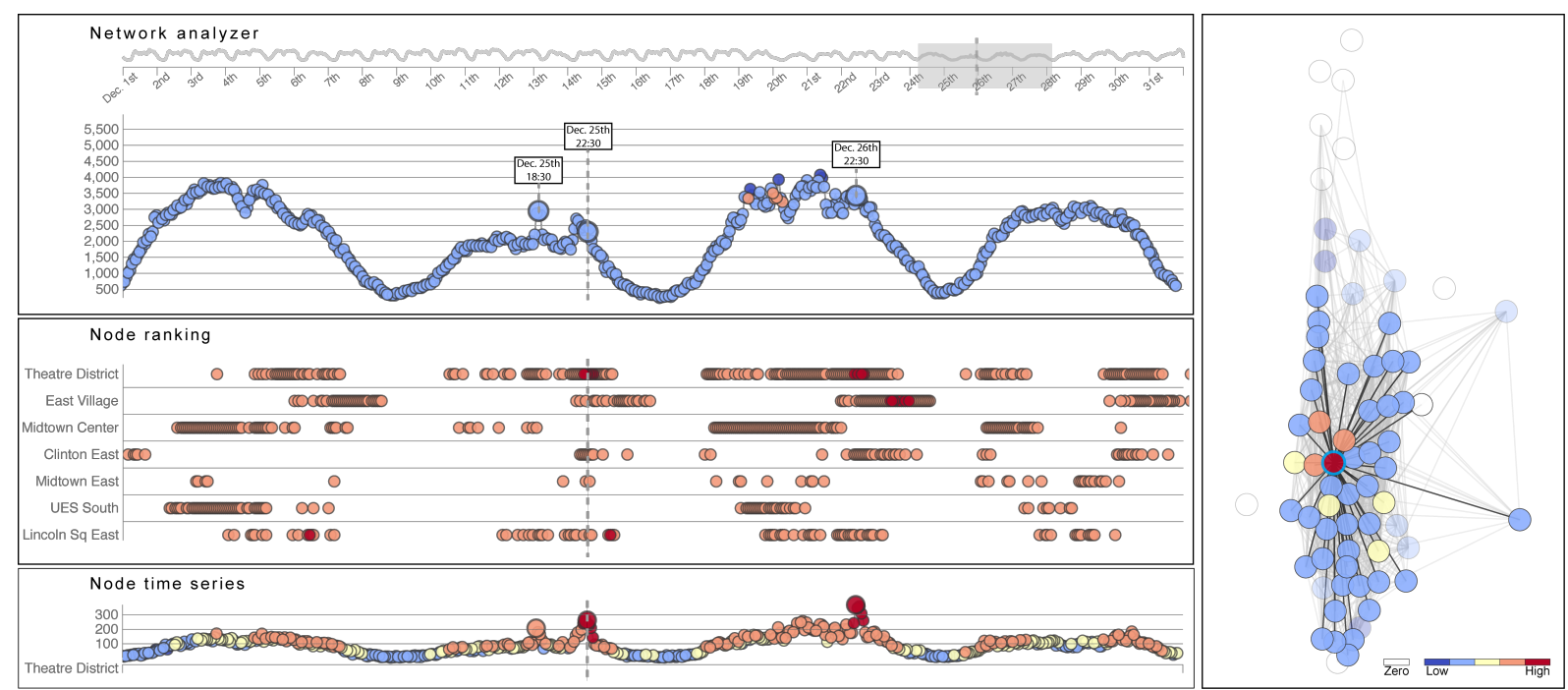

Figure 41 - Interface considering taxi trips in Manhattan in December 2009. The network analyzer presents the total number of taxi pick-ups on the period between December 24th and December 27th (zoomed-in view). In this time period, the Theater District was considered the most relevant region as shown in the node ranking. The other panels, node time series and time slice view (right), highlight this region. 


\subsection{Final Considerations \& Discussion}

This chapter presented a novel method for the visual analysis of dynamic networks. The proposed method demonstrated to be efficient in the analysis of data modeled as a dynamic network, including interpersonal contact data. This section concludes the chapter discussing important topics related to the method presented.

The representation of the most relevant class in the network analyzer does not enable access to the evolution of the frequency classes over time. A stacked graphic metaphor can be used to highlight this information, as in (VALDIVIA et al., 2015). However, our approach is still able to guide users to interesting phenomena along time, accomplishing this in a simpler way that allows to include more information, the activity level.

An important part of the current interface, the node-link diagram for the time slice view does not scale naturally to dynamic networks with a much larger number of nodes. However, the method itself is compatible with any other method that supports the encoding of at least one information, alternating the representation of the signal or the class.

Importantly, when exploring dynamic networks, usually there is interest in the detection and evolution of communities. While we believe that this problem can be approached using the graph wavelet theory, we considered it beyond the scope of this work; an interesting future work, now that we have established this methodology as a viable alternative for the visual analysis of large dynamic networks.

Another worthwhile future work is the application of our method in the analysis of dynamic multivariate networks, scenario where there are many attributes associated with the nodes and edges of a dynamic network. Once the graph wavelet transform we rely on has been developed based on unique attributes on nodes and edges, it will be necessary the development of new strategies to efficiently include additional attributes in the graph wavelet transform, since it is not straightforward to adapt the current methodology to multivariate attributes.

Finally, the possibility of choosing other signals is a concept that provides great flexibility for our method and the exploration of different options can lead to a multitude of potential new developments and applications. 
This dissertation has extensively explored the graph wavelet theory. We initially present the formulation of the theory with the aid of a solid mathematical framework, detailing proofs and developments of important formulas that have been omitted in the original publications. Motivated by a strong interest in the use of these tools, whenever possible we use illustrative examples in order to help inexperienced researchers work with these promising tools, which from our viewpoint have a huge potential still unexplored.

We've also introduced an application of the graph wavelet theory, a novel method for the visual analysis of dynamic networks. By leveraging the wavelet coefficients, our method accurately characterizes the behavior of each element of a dynamic network and uses this information to summarize the temporal evolution of the whole network, providing more information than the current state-of-the-art techniques for dynamic network exploration. We demonstrated the effectiveness and capabilities of the proposed method through comparisons and applications on real-world dynamic networks. The reduced computational cost, due to the approximation of the wavelet coefficients, enables the processing of large dynamic networks in reasonable time, which is of great importance in the present days.

Ultimately, we quantitatively emphasize the quality of the work accomplished during the development of the doctoral project. Table 2 depicts the number of full text views received by the articles directly related to the dissertation in two dissemination vehicles, IEEE Xplore <http: //ieeexplore.ieee.org > and ResearchGate <https://www.researchgate.net/>, where the articles were made available to the general public. Since they were recently published (last year), we expect even more views and possibly a significant number citations over the next few years. Additionally, we were recently invited to contribute a book chapter to be published by Springer. The invitation was based on our recent contribution "Wavelet-based visual analysis for data exploration". 
Table 2 - Full text views of the publications directly related to the dissertation. Update at March 14, 2018.

\begin{tabular}{c|c|c}
\hline Article & IEEE Xplore & ResearchGate \\
\hline \hline Wavelet-based visual analysis for data exploration & 295 & 44 \\
\hline Wavelet-based visual analysis of dynamic networks & 114 & 73 \\
\hline
\end{tabular}

\subsection{Future Work}

Beyond the interest in the use of the graph wavelet theory for other purposes, we are quite enthusiastic about another facet of graph signal processing theory, the regularization on graphs. Given a signal $f \in \mathbb{R}^{n}$ defined on the nodes of a graph $G$, a regularization on $G$ can be performed by solving the problem

$$
\underset{s}{\operatorname{argmin}}\left(\frac{\beta}{2}\|s-f\|_{2}^{2}+s^{T} L s\right)
$$

where $L$ is the graph Laplacian of graph $G$. The solution of Equation 6.1 is a smooth signal $F$ that approximates the signal $f$. The factor $s^{T} L s$ in the above equation, called graph Laplacian quadratic form of the signal $s$ with respect to the graph $G$, can be expressed as

$$
s^{T} L s=\frac{1}{2} \sum_{i=1}^{n} \sum_{j=1}^{n} w_{i j}\left[s\left(\tau_{i}\right)-s\left(\tau_{j}\right)\right]^{2} .
$$

Then, if two nodes are connected with a large weight, the signal $F$ should be similar on them to keep $F^{T} L F$ small, exactly the concept of smoothness on graphs. The parameter $\beta$ balances between signal approximation and smoothing effect. Moreover, solution $F$ has a closed formula (SHUMAN; VANDERGHEYNST; FROSSARD, 2011), it is given by

$$
F=\sum_{\ell=1}^{n}\left[\frac{\beta}{\beta+2 \lambda_{\ell}}\right] \hat{f}\left(\lambda_{\ell}\right) u_{\ell} .
$$

where $\hat{f}$ is the graph Fourier transform of signal $f$. Solution $F$ can be calculated using a fast approximation mechanism based on the Chebyshev polynomials, similar to what is done for the wavelet coefficients, enabling the application of the regularization for large graphs.

Used in the literature mainly to semi-supervised learning, we intend to use the regularization on graphs to perform multidimensional projections. Indeed, given a dataset $A$ in a high dimensional space $\mathbb{R}^{n}$, the idea is to construct a similarity graph $G$ considering instances $a \in A$ as graph nodes $g \in G$. A subset $A_{\mathbb{S}}$ of dataset $A$ is then selected. The samples in $A_{\mathbb{S}}$, called control instances, or control points, can be taken at random from $A$. A dimensionality reduction technique $\alpha$ is employed to project instances in $A_{\mathbb{S}}$, thus obtaining a set of instances $B_{\mathbb{S}}$ in $\mathbb{R}^{2}$. 
All instances $b=\alpha(a)$ in $B_{\mathbb{S}}$ have two-dimensional coordinates, namely $\alpha(a)_{x}$ and $\alpha(a)_{y}$. They are used to define two signals $f_{x}$ and $f_{y}$ on $G$ as follows

$$
\begin{aligned}
& f_{x}(a)=\left\{\begin{array}{cc}
\alpha(a)_{x} & \text { if } a \in A_{\mathbb{S}} \\
0 & \text { if } a \notin A_{\mathbb{S}}
\end{array},\right. \\
& f_{y}(a)=\left\{\begin{array}{cc}
\alpha(a)_{y} & \text { if } a \in A_{\mathbb{S}} \\
0 & \text { if } a \notin A_{\mathbb{S}}
\end{array} .\right.
\end{aligned}
$$

In other words, when node $a \in A$ belongs to subset $A_{\mathbb{S}}$, signals $f_{x}$ and $f_{y}$ attribute to $a$ value equal to its first and second coordinate, respectively, and zero otherwise. The regularization is solved using signal $f_{x}$ to obtain a smooth signal $F_{x}$. The process is repeated with $f_{y}$ to get a second smooth signal $F_{y}$. The position of an instance $a \in A$ in the new multidimensional projection method is

$$
\left(F_{x}(a), F_{y}(a)\right) \in \mathbb{R}^{2}
$$

It is important to note that the position of the control instances guilds the regularization while the smoothing effect sets the coordinates in $x$ - and $y$-axis such that neighbor instances in the high dimensional space tend to have similar coordinates in $\mathbb{R}^{2}$.

To illustrate the above concept, we consider a widely known dataset, the Iris dataset, to apply the proposed multidimensional projection. Table 3 shows general details of this dataset. We randomly select $20 \%$ of instances from Iris dataset as control samples and project them on the visual space $\mathbb{R}^{2}$ via multidimensional scaling, construct a $k$-nearest neighbor graph on the high dimensional space with $k$ equal to 5 , and set parameter $\beta$ equal to 0.25 . Figure 42 depicts the final multidimensional projection after projecting the remaining instances through the method proposed in this section.

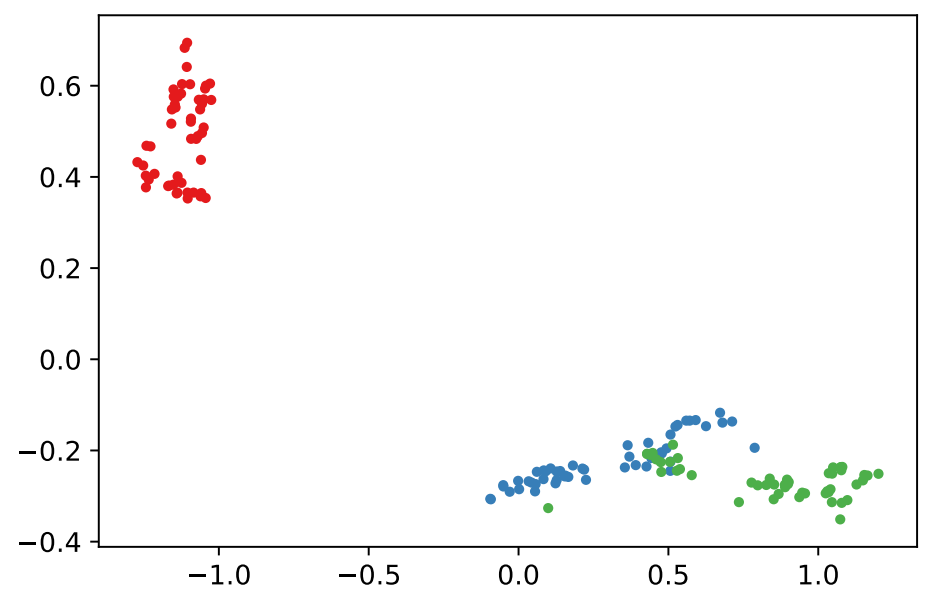

Figure 42 - Multidimensional projection of Iris dataset via regularization on graphs. 
Table 3 - General details of Iris dataset.

\begin{tabular}{c|c|c|c|c}
\hline Name & Size & Dimensionality & $\mathrm{N}^{\circ}$ of classes & Source \\
\hline \hline Iris & 150 & 4 & 3 & (LICHMAN, 2013) \\
\hline
\end{tabular}

Graph signal processing is still in its early days and there is room for much more developments and applications, which should attract even more researchers to this emerging field in the next years. 
ARCHAMBAULT, D.; ABELLO, J.; KENNEDY, J.; KOBOUROV, S.; MA, K.-L.; MIKSCH, S.; MUELDER, C.; TELEA, A. C. Temporal multivariate networks. In: Multivariate Network Visualization. [S.1.]: Springer, 2014. p. 151-174. Citation on page 36.

BACH, B.; HENRY-RICHE, N.; DWYER, T.; MADHYASTHA, T.; FEKETE, J.-D.; GRABOWSKI, T. Small MultiPiles: Piling time to explore temporal patterns in dynamic networks. Computer Graphics Forum, The Eurographics Association and John Wiley \& Sons Ltd., 2015. Citation on page 39.

BACH, B.; PIETRIGA, E.; FEKETE, J.-D. GraphDiaries: Animated transitions and temporal navigation for dynamic networks. IEEE Transactions on Visualization and Computer Graphics, v. 20, n. 5, p. 740-754, 2014. Citations on pages 36 and 37.

BACH, B.; SHI, C.; HEULOT, N.; MADHYASTHA, T.; GRABOWSKI, T.; DRAGICEVIC, P. Time Curves: Folding Time to Visualize Patterns of Temporal Evolution in Data. IEEE Transactions on Visualization and Computer Graphics, Institute of Electrical and Electronics Engineers, v. 22, n. 1, 2016. Citations on pages 40 and 41.

BECK, F.; BURCH, M.; DIEHL, S.; WEISKOPF, D. The state of the art in visualizing dynamic graphs. In: Eurographics Conference on Visualization - STARs. [S.1.]: Eurographics Association, 2014. p. 83-103. Citation on page 36.

A taxonomy and survey of dynamic graph visualization. Computer Graphics Forum, 2016. Citation on page 36.

BELKIN, M.; MATVEEVA, I.; NIYOGI, P. Regularization and semi-supervised learning on large graphs. In: SPRINGER. International Conference on Computational Learning Theory. [S.1.], 2004. v. 3120, p. 624-638. Citation on page 33.

BURCH, M.; VEHLOW, C.; BECK, F.; DIEHL, S.; WEISKOPF, D. Parallel edge splatting for scalable dynamic graph visualization. IEEE Transactions on Visualization and Computer Graphics, v. 17, n. 12, p. 2344-2353, 2011. ISSN 1077-2626. Citation on page 36.

CAO, N.; SHI, C.; LIN, S.; LU, J.; LIN, Y.-R.; LIN, C.-Y. Targetvue: Visual analysis of anomalous user behaviors in online communication systems. IEEE Transactions on Visualization and Computer Graphics, Institute of Electrical and Electronics Engineers, v. 22, n. 1, p. 280-289, 2016. Citation on page 40.

CHUNG, F. R. Spectral graph theory. [S.1.]: American Mathematical Soc., 1997. Citation on page 43 .

CROVELLA, M.; KOLACZYK, E. Graph wavelets for spatial traffic analysis. In: Joint Conference of the IEEE Computer and Communications Societies. [S.1.]: Institute of Electrical and Electronics Engineers, 2003. v. 3, p. 1848-1857. ISBN 0-7803-7752-4. Citations on pages 33 and 34. 
CUI, W.; WANG, X.; LIU, S.; RICHE, N. H.; MADHYASTHA, T. M.; MA, K. L.; GUO, B. Let it flow: a static method for exploring dynamic graphs. In: INSTITUTE OF ELECTRICAL AND ELECTRONICS ENGINEERS. IEEE Pacific Visualization Symposium. [S.1.], 2014. p. 121-128. Citations on pages 39 and 40 .

DAL COL, A.; VAlDIVIA, P.; PETRONETTO, F.; DIAS, F.; SILVA, C. T.; NONATO, L. G. Wavelet-based visual analysis for data exploration. Computing in Science \& Engineering, Institute of Electrical and Electronics Engineers, v. 19, n. 5, p. 85-91, 2017. No citation.

Wavelet-based visual analysis of dynamic networks. IEEE Transactions on Visualization and Computer Graphics, Institute of Electrical and Electronics Engineers, 2017. No citation.

DANG, T. N.; PENDAR, N.; FORBES, A. G. Timearcs: Visualizing fluctuations in dynamic networks. Computer Graphics Forum, v. 35, n. 3, p. 61-69, 2016. ISSN 1467-8659. Citations on pages $36,38,78$, and 84 .

DIEHL, S.; GÖRG, C. Graphs, they are changing - dynamic graph drawing for a sequence of graphs. In: Graph Drawing. [S.1.]: Springer-Verlag, 2002, (GD). p. 23-31. ISBN 3-540-00158-1. Citation on page 36.

ELZEN, S. van den; HOLTEN, D.; BLAAS, J.; WIJK, J. J. van. Dynamic network visualization with extended massive sequence views. IEEE Transactions on Visualization and Computer Graphics, v. 20, n. 8, p. 1087-1099, 2014. ISSN 1077-2626. Citations on pages 36 and 38.

Reducing snapshots to points: A visual analytics approach to dynamic network exploration. IEEE Transactions on Visualization and Computer Graphics, Institute of Electrical and Electronics Engineers, v. 22, n. 1, p. 1-10, 2016. Citations on pages 40, 41, 77, 81, and 83.

ERTEN, C.; HARDING, P. J.; KOBOUROV, S. G.; WAMPLER, K.; YEE, G. V. GraphAEL: Graph animations with evolving layouts graph drawing. In: Graph Drawing. [S.1.]: Springer, 2004. p. 98-110. ISBN 978-3-540-20831-0. Citations on pages 36 and 37.

FARRUGIA, M.; HURLEY, N.; QUIGLEY, A. Exploring temporal ego networks using small multiples and tree-ring layouts. International Conference on Advances in Computer-Human Interactions, v. 2011, p. 23-28, 2011. Citations on pages 37 and 38.

FOURNET, J.; BARRAT, A. Contact patterns among high school students. PLoS ONE, Public Library of Science, v. 9, n. 9, p. e107878, 2014. Citations on pages 80 and 81.

FRIEDRICH, C.; EADES, P. Graph drawing in motion. Journal of Graph Algorithms and Applications, v. 6, n. 3, p. 353-370, 2002. Citations on pages 36 and 37.

FRUCHTERMAN, T. M.; REINGOLD, E. M. Graph drawing by force-directed placement. Software: Practice and Experience, Wiley Online Library, v. 21, n. 11, p. 1129-1164, 1991. Citation on page 76 .

FUCHS, J.; FISCHER, F.; MANSMANN, F.; BERTINI, E.; ISENBERG, P. Evaluation of alternative glyph designs for time series data in a small multiple setting. In: ACM. SIGCHI Conference on Human Factors in Computing Systems. [S.1.], 2013. p. 3237-3246. Citations on pages 36 and 37.

GHONIEM, M.; FEKETE, J.-D.; CASTAGLIOLA, P. A comparison of the readability of graphs using node-link and matrix-based representations. In: IEEE Symposium on Information Visualization. [S.1.: s.n.], 2004. p. 17-24. Citation on page 39. 
GLEICH, D. The matlabbgl matlab library. 2015. Citation on page 64.

GOVE, R.; GRAMSKY, N.; KIRBY, R.; SEFER, E.; SOPAN, A.; DUNNE, C.; SHNEIDERMAN, B.; TAIEB-MAIMON, M. Netvisia: Heat map \& matrix visualization of dynamic social network statistics \& content. In: INSTITUTE OF ELECTRICAL AND ELECTRONICS ENGINEERS. International Conference on Privacy, Security, Risk, and Trust, IEEE International Conference on Social Computing. [S.1.], 2011. p. 19-26. Citation on page 37.

HADLAK, S.; SCHUMANN, H.; CAP, C. H.; WOLLENBERG, T. Supporting the visual analysis of dynamic networks by clustering associated temporal attributes. IEEE Transactions on Visualization and Computer Graphics, v. 19, n. 12, p. 2267-2276, 2013. ISSN 1077-2626. Citations on pages 39 and 40.

HADLAK, S.; SCHUMANN, H.; SCHULZ, H.-J. A survey of multi-faceted graph visualization. In: Eurographics Conference on Visualization - STARs. [S.1.]: The Eurographics Association, 2015. Citation on page 36.

HAMMOND, D. K.; VANDERGHEYNST, P.; GRIBONVAL, R. Wavelets on graphs via spectral graph theory. Applied and Computational Harmonic Analysis, Elsevier, v. 30, n. 2, p. 129 150, 2011. Citations on pages 27, 28, 29, 33, 34, 43, 48, 50, 52, 58, 60, and 101.

HEIL, C. E.; WALNUT, D. F. Continuous and discrete wavelet transforms. Society for Industrial and Applied Mathematics Review, SIAM, v. 31, n. 4, p. 628-666, 1989. Citation on page 60 .

HILBERT, M.; LÓPEZ, P. The world's technological capacity to store, communicate, and compute information. Science, American Association for the Advancement of Science, v. 332, n. 6025 , p. 60-65, 2011. Citation on page 27.

KEIM, D.; ANDRIENKO, G.; FEKETE, J.-D.; GÖRG, C.; KOHLHAMMER, J.; MELANÇON, G. Visual analytics: Definition, process, and challenges. In: Information Visualization. [S.1.]: Springer, 2008. p. 154-175. Citation on page 28.

LANDESBERGER, T. von; DIEL, S.; BREMM, S.; FELLNER, D. W. Visual analysis of contagion in networks. Information Visualization, SAGE Publications, v. 14, n. 2, p. 93-110, 2015. Citation on page 40.

LICHMAN, M. UCI Machine Learning Repository. 2013. Available: < http://archive.ics.uci. edu/ml>. Citation on page 92.

MILLER, B. A.; BEARD, M. S.; WOLFE, P. J.; BLISS, N. T. A spectral framework for anomalous subgraph detection. IEEE Transactions on Signal Processing, v. 63, n. 16, p. 41914206, 2015. ISSN 1053-587X. Citation on page 33.

MOHAN, D. M.; ASIF, M. T.; MITROVIC, N.; DAUWELS, J.; JAILLET, P. Wavelets on graphs with application to transportation networks. In: IEEE International Conference on Intelligent Transportation Systems. [S.1.]: Institute of Electrical and Electronics Engineers, 2014. p. 1707-1712. ISBN 978-1-4799-6078-1. Citations on pages 28, 34, and 35.

NARANG, S. K.; ORTEGA, A. Perfect reconstruction two-channel wavelet filter banks for graph structured data. IEEE Transactions on Signal Processing, Institute of Electrical and Electronics Engineers, v. 60, n. 6, p. 2786-2799, 2012. Citation on page 44. 
PERRAUDIN, N.; PARATTE, J.; SHUMAN, D.; MARTIN, L.; KALOFOLIAS, V.; VANDERGHEYNST, P.; HAMMOND, D. K. Gspbox: A toolbox for signal processing on graphs. arXiv:1408.5781, 2014. Citation on page 33.

SANDRYHAILA, A.; MOURA, J. M. Classification via regularization on graphs. In: IEEE Global Conference on Signal and Information Processing. [S.1.: s.n.], 2013. p. 495-498. Citation on page 33.

Discrete signal processing on graphs. IEEE Transactions on Signal Processing, Institute of Electrical and Electronics Engineers, v. 61, n. 7, p. 1644-1656, 2013. Citations on pages 28 and 33 .

Big data analysis with signal processing on graphs: Representation and processing of massive data sets with irregular structure. IEEE Signal Processing Magazine, Institute of Electrical and Electronics Engineers, v. 31, n. 5, p. 80-90, 2014. Citations on pages 34, 43, 44, 65 , and 73 .

SARAIYA, P.; LEE, P.; NORTH, C. Visualization of graphs with associated timeseries data. In: INSTITUTE OF ELECTRICAL AND ELECTRONICS ENGINEERS. IEEE Symposium on Information Visualization. [S.1.], 2005. p. 225-232. Citations on pages 36 and 37.

SHUMAN, D. I.; NARANG, S. K.; FROSSARD, P.; ORTEGA, A.; VANDERGHEYNST, P. The emerging field of signal processing on graphs: Extending high-dimensional data analysis to networks and other irregular domains. IEEE Signal Processing Magazine, Institute of Electrical and Electronics Engineers, v. 30, n. 3, p. 83-98, 2013. Citations on pages 27, 33, and 47.

SHUMAN, D. I.; RICAUD, B.; VANDERGHEYNST, P. A windowed graph fourier transform. In: INSTITUTE OF ELECTRICAL AND ELECTRONICS ENGINEERS. Statistical Signal Processing Workshop. [S.1.], 2012. p. 133-136. Citation on page 47.

. Vertex-frequency analysis on graphs. Applied and Computational Harmonic Analysis, Elsevier, v. 40, n. 2, p. 260-291, 2016. Citation on page 33.

SHUMAN, D. I.; VANDERGHEYNST, P.; FROSSARD, P. Chebyshev polynomial approximation for distributed signal processing. In: INSTITUTE OF ELECTRICAL AND ELECTRONICS ENGINEERS. IEEE International Conference on Distributed Computing in Sensor Systems and Workshops. [S.1.], 2011. p. 1-8. Citations on pages 27, 34, 54, and 90.

SHUMAN, D. I.; WIESMEYR, C.; HOLIGHAUS, N.; VANDERGHEYNST, P. Spectrumadapted tight graph wavelet and vertex-frequency frames. IEEE Transactions on Signal Processing, Institute of Electrical and Electronics Engineers, v. 63, n. 16, p. 4223-4235, 2015. Citations on pages 28, 33, 58, and 60 .

SINGH, R.; CHAKRABORTY, A.; MANOJ, B. Gft centrality: A new node importance measure for complex networks. Physica A: Statistical Mechanics and its Applications, Elsevier, v. 487, p. 185-195, 2017. Citation on page 33.

SPIELMAN, D. A. Spectral graph theory and its applications. In: INSTITUTE OF ELECTRICAL AND ELECTRONICS ENGINEERS. 48th Annual IEEE Symposium on Foundations of Computer Science. [S.1.], 2007. p. 29-38. Citation on page 43. 
STEIGER, M.; BERNARD, J.; MITTELSTÄDT, S.; LÜCKE-TIEKE, H.; KEIM, D.; MAY, T.; KOHLHAMMER, J. Visual analysis of time-series similarities for anomaly detection in sensor networks. In: WILEY ONLINE LIBRARY. Computer Graphics Forum. [S.1.], 2014. v. 33, p. 401-410. Citation on page 40.

STEIN, K.; WEGENER, R.; SCHLIEDER, C. Pixel-oriented visualization of change in social networks. In: INSTITUTE OF ELECTRICAL AND ELECTRONICS ENGINEERS. IEEE International Conference on Advances in Social Networks Analysis and Mining. [S.1.], 2010. p. 233-240. Citations on pages 36 and 39.

SUSNJARA, A.; PERRAUDIN, N.; KRESSNER, D.; VANDERGHEYNST, P. Accelerated filtering on graphs using lanczos method. arXiv:1509.04537, 2015. Citation on page 34.

TREMBLAY, N.; BORGNAT, P. Graph wavelets for multiscale community mining. IEEE Transactions on Signal Processing, v. 62, n. 20, p. 5227-5239, 2014. ISSN 1053-587X. Citations on pages $28,34,35$, and 50 .

VALDIVIA, P.; DIAS, F.; PETRONETTO, F.; SILVA, C. T.; NONATO, L. Wavelet-based visualization of time-varying data on graphs. In: INSTITUTE OF ELECTRICAL AND ELECTRONICS ENGINEERS. IEEE Conference on Visual Analytics Science and Technology. [S.1.], 2015. Citations on pages 27, 28, 34, 35, 73, and 88.

VEHLOW, C.; BECK, F.; WEISKOPF, D. The state of the art in visualizing group structures in graphs. In: Eurographics Conference on Visualization - STARs. [S.1.]: The Eurographics Association, 2015. Citation on page 36.

Visualizing dynamic hierarchies in graph sequences. IEEE Transactions on Visualization and Computer Graphics, v. 22, n. 10, p. 2343-2357, 2016. ISSN 1077-2626. Citation on page 36 .

YI, J. S.; ELMQVIST, N.; LEE, S. Timematrix: Analyzing temporal social networks using interactive matrix-based visualizations. International Journal of Human-Computer Interaction, Taylor \& Francis, v. 26, n. 11-12, p. 1031-1051, 2010. Citation on page 36.

ZHANG, C.; FLORÊNCIO, D.; LOOP, C. Point cloud attribute compression with graph transform. In: IEEE International Conference on Image Processing. [S.1.]: Institute of Electrical and Electronics Engineers, 2014. p. 2066-2070. Citation on page 33.

ZHOU, D.; SCHÖLKOPF, B. A regularization framework for learning from graph data. In: ICML Workshop on Statistical Relational Learning and its Connections to Other Fields. [S.1.: s.n.], 2004. v. 15, p. 67-68. Citation on page 33.

ZHOU, D.; WESTON, J.; GRETTON, A.; BOUSQUET, O.; SCHÖLKOPF, B. Ranking on data manifolds. In: Advances in Neural Information Processing Systems. [S.1.: s.n.], 2004. p. 169-176. Citation on page 33. 

APPENDIX

A

DEMONSTRATIONS

In this appendix, we present some important demonstrations for the dissertation.

Proof of the recursive formula (Equation 3.39):

$$
\bar{T}_{k}(L) f=\frac{2}{a}(L-a I) \bar{T}_{k-1}(L) f-\bar{T}_{k-2}(L) f,
$$

for $2 \leq k \leq m$.

\section{Proof:}

$$
\begin{aligned}
\bar{T}_{k}(L) f & =T_{k}\left(\frac{L-a I}{a}\right) f \\
\stackrel{(3.37)}{=} & {\left[2\left(\frac{L-a I}{a}\right) T_{k-1}\left(\frac{L-a I}{a}\right)-T_{k-2}\left(\frac{L-a I}{a}\right)\right] f } \\
& =\frac{2}{a}(L-a I) T_{k-1}\left(\frac{L-a I}{a}\right) f-T_{k-2}\left(\frac{L-a I}{a}\right) f \\
& =\frac{2}{a}(L-a I) \bar{T}_{k-1}(L) f-\bar{T}_{k-2}(L) f .
\end{aligned}
$$

Proof of the initial conditions (Equation 3.40):

$$
\begin{aligned}
& \bar{T}_{0}(L) f=f \\
& \bar{T}_{1}(L) f=\frac{1}{a}(L-a I) f .
\end{aligned}
$$

Proof: 


$$
\begin{aligned}
\bar{T}_{0}(L) f & =T_{0}\left(\frac{L-a I}{a}\right) f \\
& =I \cdot f \\
& =f \\
\bar{T}_{1}(L) f & =T_{1}\left(\frac{L-a I}{a}\right) f \\
& =\frac{L-a I}{a} \cdot f \\
& =\frac{1}{a}(L-a I) f .
\end{aligned}
$$

Proof of Equation 3.41:

$$
\bar{T}_{k}(L) f=\sum_{\ell=1}^{n} \bar{T}_{k}\left(\lambda_{\ell}\right) \hat{f}\left(\lambda_{\ell}\right) u_{\ell}
$$

Proof: The demonstration is made by induction over the integer $k$. Firstly, let's prove the equality for $k=0$. Using the inverse graph Fourier transform of $f$

$$
\begin{aligned}
\bar{T}_{0}(L) f & =f \\
& =\sum_{\ell=1}^{n} \hat{f}\left(\lambda_{\ell}\right) u_{\ell} \\
& =\sum_{\ell=1}^{n} \bar{T}_{0}\left(\lambda_{\ell}\right) \hat{f}\left(\lambda_{\ell}\right) u_{\ell},
\end{aligned}
$$

since $\bar{T}_{0}\left(\lambda_{\ell}\right)=T_{0}\left(\frac{\lambda_{\ell}-a}{a}\right)=1$. In order to prove that the equality holds for $k=1$, we recall the following expression to $L f$

$$
\begin{aligned}
L f & =L \sum_{\ell=1}^{n} \hat{f}\left(\lambda_{\ell}\right) u_{\ell} \\
& =\sum_{\ell=1}^{n} \hat{f}\left(\lambda_{\ell}\right) L u_{\ell} \\
& =\sum_{\ell=1}^{n} \lambda_{\ell} \hat{f}\left(\lambda_{\ell}\right) u_{\ell}
\end{aligned}
$$

where the last equality is thanks to the fact that $u_{\ell}$ is an eigenvector of the graph Laplacian with corresponding eigenvalue $\lambda_{\ell}\left(L u_{\ell}=\lambda_{\ell} u_{\ell}\right)$. Therefore, 


$$
\begin{aligned}
\bar{T}_{1}(L) f & =\frac{1}{a}(L-a I) f \\
& =\frac{1}{a}(L f-a f) \\
& =\frac{1}{a}\left(\sum_{\ell=1}^{n} \lambda_{\ell} \hat{f}\left(\lambda_{\ell}\right) u_{\ell}-a \sum_{\ell=1}^{n} \hat{f}\left(\lambda_{\ell}\right) u_{\ell}\right) \\
& =\sum_{\ell=1}^{n} \frac{1}{a}\left(\lambda_{\ell}-a\right) \hat{f}\left(\lambda_{\ell}\right) u_{\ell} \\
& =\sum_{\ell=1}^{n} \bar{T}_{1}\left(\lambda_{\ell}\right) \hat{f}\left(\lambda_{\ell}\right) u_{\ell},
\end{aligned}
$$

since $\bar{T}_{1}\left(\lambda_{\ell}\right)=T_{1}\left(\frac{\lambda_{\ell}-a}{a}\right)=\frac{1}{a}\left(\lambda_{\ell}-a\right)$, ensuring the equality for $k=1$. Finally, we suppose that the equality is true for the integers $0,1, \ldots, k-1$ and prove the equality for $k$

$$
\begin{aligned}
\bar{T}_{k}(L) f \stackrel{(3.39)}{=} \frac{2}{a}(L-a I) \bar{T}_{k-1}(L) f-\bar{T}_{k-2}(L) f \\
=\frac{2}{a}(L-a I) \sum_{\ell=1}^{n} \bar{T}_{k-1}\left(\lambda_{\ell}\right) \hat{f}\left(\lambda_{\ell}\right) u_{\ell}-\sum_{\ell=1}^{n} \bar{T}_{k-2}\left(\lambda_{\ell}\right) \hat{f}\left(\lambda_{\ell}\right) u_{\ell} \\
\quad=\sum_{\ell=1}^{n}\left[\frac{2}{a}\left(\lambda_{\ell}-a\right) \bar{T}_{k-1}\left(\lambda_{\ell}\right)-\bar{T}_{k-2}\left(\lambda_{\ell}\right)\right] \hat{f}\left(\lambda_{\ell}\right) u_{\ell} \\
\stackrel{(3.37)}{=} \sum_{\ell=1}^{n} \bar{T}_{k}\left(\lambda_{\ell}\right) \hat{f}\left(\lambda_{\ell}\right) u_{\ell},
\end{aligned}
$$

concluding the induction. Note that, we used again the fact that $u_{\ell}$ is an eigenvector of the graph Laplacian $L$.

Proof of the approximation of the scaling function coefficient (Equation 3.48):

$$
S_{f}(\tau) \approx\left(\frac{1}{2} d_{0} f+\sum_{k=1}^{m} d_{k} \bar{T}_{k}(L) f\right)(\tau),
$$

Proof: Since the shifted Chebyshev polynomials form an orthogonal basis for the Hilbert space of square integrable functions defined on the interval $\left[0, \lambda_{n}\right]$ (HAMMOND; VANDERGHEYNST; GRIBONVAL, 2011), we may write

$$
\hat{h}(\lambda)=\frac{1}{2} d_{0}+\sum_{k=1}^{\infty} d_{k} \bar{T}_{k}(\lambda)
$$

where $d_{k}=\frac{2}{\pi} \int_{0}^{\pi} \cos (k \theta) \hat{h}(a(\cos (\theta)+1)) d \theta$. Then, the scaling function coefficient on a node $\tau$ can be approximated as 


$$
\begin{aligned}
S_{f}(\tau) & =\sum_{\ell=1}^{n} \hat{h}\left(\lambda_{\ell}\right) \hat{f}\left(\lambda_{\ell}\right) u_{\ell}(\tau) \\
& \stackrel{(A .1)}{=} \sum_{\ell=1}^{n}\left[\frac{1}{2} d_{0}+\sum_{k=1}^{\infty} d_{k} \bar{T}_{k}\left(\lambda_{\ell}\right)\right] \hat{f}\left(\lambda_{\ell}\right) u_{\ell}(\tau) \\
& \approx \sum_{\ell=1}^{n}\left[\frac{1}{2} d_{0}+\sum_{k=1}^{m} d_{k} \bar{T}_{k}\left(\lambda_{\ell}\right)\right] \hat{f}\left(\lambda_{\ell}\right) u_{\ell}(\tau) \\
& =\frac{1}{2} d_{0} \sum_{\ell=1}^{n} \hat{f}\left(\lambda_{\ell}\right) u_{\ell}(\tau)+\sum_{k=1}^{m} d_{k} \sum_{\ell=1}^{n} \bar{T}_{k}\left(\lambda_{\ell}\right) \hat{f}\left(\lambda_{\ell}\right) u_{\ell}(\tau) \\
& \stackrel{(3.41)}{=}\left(\frac{1}{2} d_{0} f+\sum_{k=1}^{m} d_{k} \bar{T}_{k}(L) f\right)(\tau),
\end{aligned}
$$

finalizing the proof. 


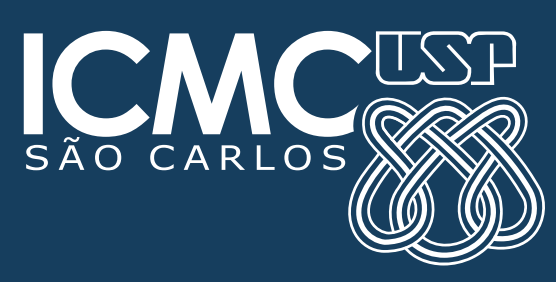

